\title{
Three-loop master integrals for ladder-box diagrams with one massive leg
}

\author{
Stefano Di Vita, ${ }^{a}$ Pierpaolo Mastrolia, ${ }^{a, b}$ Ulrich Schubert ${ }^{a}$ and Valery Yundin $^{a}$ \\ ${ }^{a}$ Max-Planck-Institut für Physik, \\ Föhringer Ring 6, D-80805 München, Germany \\ ${ }^{b}$ Dipartimento di Fisica e Astronomia, Università di Padova and \\ INFN Sezione di Padova, \\ Via Marzolo 8, I-35131 Padova, Italy \\ E-mail: divita@mpp.mpg.de, pierpaolo.mastrolia@cern.ch, \\ schubert@mpp.mpg.de, yundin@mpp.mpg.de
}

ABSTRACT: The three-loop master integrals for ladder-box diagrams with one massive leg are computed from an eighty-five by eighty-five system of differential equations, solved by means of Magnus exponential. The results of the considered box-type integrals, as well as of the tower of vertex- and bubble-type master integrals associated to subtopologies, are given as a Taylor series expansion in the dimensional regulator parameter $\epsilon=(4-d) / 2$. The coefficients of the series are expressed in terms of uniform weight combinations of multiple polylogarithms and transcendental constants up to weight six. The considered integrals enter the next-to-next-to-next-to-leading order virtual corrections to scattering processes like the three-jet production mediated by vector boson decay, $V^{*} \rightarrow j j j$, as well as the Higgs plus one-jet production in gluon fusion, $p p \rightarrow H j$.

Keywords: NLO Computations

ARXIV EPRINT: 1408.3107 


\section{Contents}

1 Introduction 1

2 Differential equations and Magnus exponential 5

2.1 Magnus exponential 6

$\begin{array}{lll}2.2 & \text { Canonical transformation } & 7\end{array}$

2.3 Canonical system 8

$\begin{array}{lll}3 & \text { Two-loop master integrals } & 10\end{array}$

4 Three-loop master integrals $\quad 12$

5 Boundary conditions $\quad \mathbf{1 5}$

$\begin{array}{lll}5.1 & \text { Relations for one-scale integrals } & 15\end{array}$

$\begin{array}{llr}6 & \text { Conclusions } & 18\end{array}$

$\begin{array}{lr}\text { A Canonical matrices at two-loop } & 19\end{array}$

$\begin{array}{ll}\text { B Canonical matrices at three-loop } & 21\end{array}$

$\begin{array}{ll}\text { C Three-loop scalar ladder } & 29\end{array}$

\section{Introduction}

Feynman integrals are the building blocks for describing particle interactions beyond the tree approximation in perturbation theory. When their direct integration becomes prohibitive, because of either the number of loops or of external legs, the evaluation of Feynman integrals can be addressed in two phases, namely the decomposition in terms of basic integrals, followed by the evaluation of the latter, to be considered as an easier problem.

Within the continuous dimensional regularization scheme, Feynman integrals fulfill identities that fall in the category of the general class of integration-by-parts relations [13]. Such relations can be exploited in order to identify a set of independent integrals, dubbed master integrals (MI's), that can be used as a basis of functions for the virtual contributions to scattering amplitudes. Therefore, for any given process, it is possible to identify a minimal set of MI's, such that all Feynman integrals contributing to it admit a representation in terms of the chosen basis of MI's.

Scattering amplitudes are combinations of Feynman diagrams. Therefore, it is not unnatural that global properties of scattering amplitudes can be exploited to achieve the 
decomposition of the whole amplitude in terms of MI's. In fact, unitarity and factorization, both at the integral and integrand level, become suitable tools for the simultaneous decomposition of group of diagrams in terms of MI's [4-6] (see [7, 8] for reviews). Decomposing the whole amplitude, rather than the individual diagrams, has the advantage that at each step of the computation the coefficients of the decomposition reflect the symmetries of the amplitude. Whereas, the decomposition of each integral separately may not carry such information, with the drawback of introducing spurious terms at the intermediate steps of the calculation, which necessarily disappear from the total result.

The advantage of decomposing amplitudes, rather than individual integrals, in terms of MI's clearly emerged in the last decade, as it has been the driving principle determining the breakthrough in the evaluation of next-to-leading order (NLO) virtual corrections to multi-leg processes (see for instance [9] and references therein). Activities are ongoing to extend the underlying ideas to higher orders [10-15].

The evaluation of MI's usually proceeds one-by-one in a bottom up approach, starting from the less complicated integrals and systematically enriching their structure by increasing the number of internal and external lines. MI's are functions of the kinematic invariants built with the external momenta and of the masses of the involved particles. Remarkably, the integration-by-parts (IBP) relations imply that the MI's obey linear systems of firstorder differential equations (DE's) in the kinematic invariants, which can be used for the determination of their actual analytic expressions [16-18].

The simplicity of the whole amplitude with respect to the individual integrals is reflected also in the fact that the analytic functions present in each MI do not necessarily appear in the final result, obtained after combining coefficients and MI's altogether. Remarkably, Hopf algebra structure (of multiple polylogarithms) can be used to simplify complicated expressions for multi-loop amplitudes [19], or global symmetries can be used to constrain the minimal set of variables an amplitude may depend on [20]. In other words, integrals depend on the kinematic invariants enforced by their external topology and masses, but amplitudes may depend on functions of such invariants, which resolve the symmetries that are not manifest at the diagrammatic level.

In this spirit, the role of differential equations is twofold, because they are not only a tool for evaluating individual integrals, but also the location where investigating how the exposed analytic properties of MI's could be transferred to amplitudes.

For any given scattering process the set of MI's is not unique [21]. Rather than considering it a limitation, this freedom can be exploited in order to select the basis of MI's that can be easier to evaluate. In the context of the differential equations method [16$18]$, reviewed in [22, 23], and further developed in [24, 25], simplifying the evaluation of MI's means achieving a block-triangular form of the system where: i) the dimensions of the blocks are minimal; ii) all analytic properties are exposed; iii) the solution can be determined through an algebraic procedure.

According to the study in ref. [24], generic systems of DE's for MI's whose associated matrix depends on rational functions of the kinematics and $\epsilon$ could be brought in a canonical 


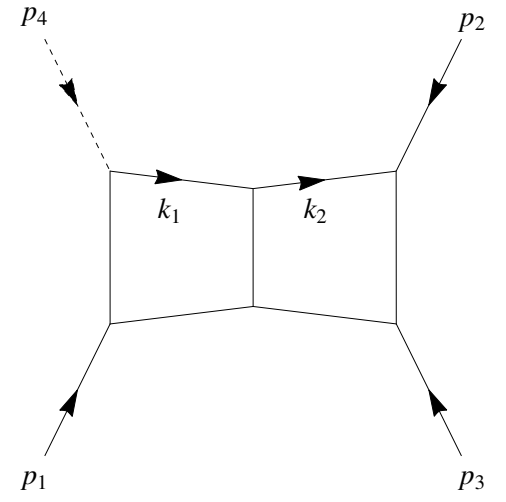

(a)

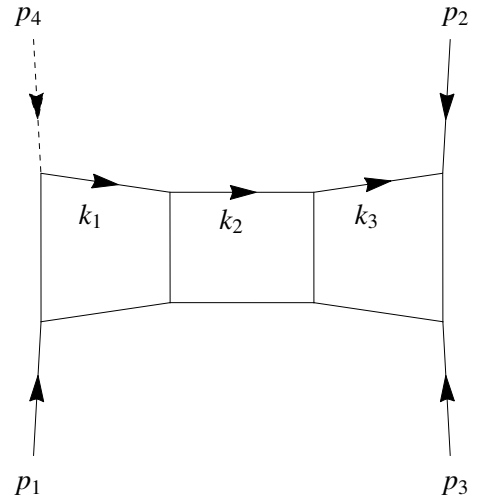

(b)

Figure 1. The two-loop and three-loop ladder box diagram, with one off-shell leg: the solid lines stand for massless particles; the dashed line represents a massive particle. Momentum conservation is $\sum_{i=1}^{4} p_{i}=0$, with $p_{i}^{2}=0(i=1,2,3)$ and $p_{4}^{2}=m^{2}$.

form where the $\epsilon$-dependence is factorized from the kinematic. The integration of a canonical system of DE's can be carried out with algebraic patterns, where the analytic properties of its solution are manifestly inherited from the matrix associated to the system, which becomes the kernel of the representation of the solution in terms of repeated integrations [26]. These novel ideas for evaluating MI's have recently stimulated several applications [27-37].

In ref. [31], we proposed to address the evaluation of MI's by means of a unitary-like formalism, where Magnus exponential [38] is responsible for the kinematic evolution of the MI's by acting on the boundary conditions. In particular, we observed that an initial set of MI's obeying a systems of DE's which depends linearly on $\epsilon$ can be brought in canonical form after applying a change of basis by means of a transformation that absorbs the constant term. The change from the initial set to the canonical basis of MI's can be implemented with Magnus exponential [38, 39]. The solution admits an $\epsilon$-expansion in terms of Dyson or equivalently Magnus series, whose coefficients are written as nested integrations [26, 40-44].

In this article, we apply Magnus exponential method to solve the system of differential equations fulfilled by 85 MI's required for the determination of the three-loop ladder box integrals with one massive leg, shown in figure $1 \mathrm{~b}$, and of the tower of integrals associated to their subtopologies, depicted in figures $3-5$. All propagators are massless. The solution of the system is finally determined after fixing the values of the otherwise arbitrary constants that naturally arise from solving differential equations. In the considered case, boundary conditions are obtained by imposing the regularity of the MI's around unphysical singularities, ruling out the divergent behavior of the general solution of the systems.

Among the evaluated 85 MI's, there are 16 vertex-like integrals with two off-shell legs not yet considered in the literature, and 10 one-scale vertex- and bubble-like integrals, which 
had already been computed by means of Mellin-Barnes integral representation [45-48]. In general, homogeneous differential equations for single scale integrals carry only information on the scaling behaviour of the solution. The determination of the boundary constants for such differential equations amounts to the evaluation of the integrals themselves by other means. Within a multi-scale problem, where integrals may depend on more than one external invariant, single-scale integrals enter the regularity conditions (or equivalently could be the limit) of the multi-scale ones [28, 49]. These relations, entangling single- and multi-scale functions, can be exploited to determine the arbitrary constants of the singlescale integrals, or at least to reduce the number of independent single-scale integrals that needs to be computed by alternative methods, other than differential equations. Therefore, solving multi-scale systems of differential equations yields the simultaneous determination of single- and multi-scale MI's, which are finally expressed in terms of a few single-scale MI's, to be independently provided. In fact, the considered case of 85 MI's requires only 2 one-scale integrals as external input.

The MI's hereby computed can be considered the first contributions to the next-tonext-to-next-to-leading order $\left(\mathrm{N}^{3} \mathrm{LO}\right)$ virtual corrections to scattering processes like the three-jet production from vector boson decay, $V^{*} \rightarrow j j j$, as well as the Higgs plus one-jet production in gluon fusion, $p p \rightarrow H j$, currently computed at NNLO accuracy in refs. [5053] and refs. [54, 55] respectively. The collinear limits of the MI's we present enter the computation of the three-loop one-particle splitting amplitudes, currently known at twoloop order [56]. Such amplitudes also serve as an ingredient for the derivation, following the procedure of ref. [57], of the $\mathrm{N}^{3} \mathrm{LO}$ Altarelli-Parisi splitting kernel.

The results of the considered box-type integrals and of the tower of vertex- and bubbletype integrals associated to subtopologies are given as a Taylor series expansion in $\epsilon$. The coefficients of the series are expressed in terms of uniform weight combinations of transcendental constants and generalised harmonic polylogarithms [41, 42, 58] up to weight 6 .

We also present the calculation of the two-loop one-mass planar box diagram in figure 1a, giving the result for the corresponding MI's, whose topologies are depicted in figure 3. We provide higher orders $\epsilon$-expansions w.r.t. the expressions available in the literature $[18,59]$.

We used the computer code Reduze $2[60,61]$ for solving the system of integration-byparts relations and generating the system of differential equations. The analytic results of the MI's at two- and three-loop can be numerically evaluated by means of GiNaC [43, 62] and are found in agreement with the outcome of the direct numerical integrations carried out by FIESTA 3 [63, 64] (within per-mille level of accuracy).

The paper is organized as follows. In section 2, we discuss the generic form of the systems of differential equations which are required to compute the ladder box integrals with one massive-leg at two- and three-loop. There we show how the canonical systems are constructed by using Magnus exponential matrices. In sections 3 and 4, we present the canonical bases for the two- and three-loop cases respectively. In section 5, the limiting conditions for fixing the boundary are explained, showing how meaningful relations for single-scale integrals emerge. 
In the appendices $\mathrm{A}$ and $\mathrm{B}$, we collect the representative matrices of the canonical systems. appendix C contains the analytic expression of the three-loop one-mass scalarbox integral.

The sets of 18 two-loop MI's and of 85 three-loop MI's are collected in two ancillary files 2loopMIs.m and 3loopMIs.m available from http://arxiv.org/abs/1408.3107.

\section{Differential equations and Magnus exponential}

All Feynman integrals belonging to the topologies which we consider in the present work can be defined in terms of the following sets of denominators $\mathcal{D}_{n}\left(k_{i}\right.$ are the loop momenta). For the two-loop ladder diagram in figure 1a, one has

$$
\begin{array}{lll}
\mathcal{D}_{1}=k_{1}^{2}, \quad \mathcal{D}_{2}=k_{2}^{2}, & \mathcal{D}_{3}=\left(k_{1}-k_{2}\right)^{2}, & \mathcal{D}_{4}=\left(k_{2}+p_{2}\right)^{2}, \\
\mathcal{D}_{5}=\left(k_{1}+p_{2}+p_{3}\right)^{2}, & \mathcal{D}_{6}=\left(k_{2}+p_{2}+p_{3}\right)^{2}, & \mathcal{D}_{7}=\left(k_{1}+p_{1}+p_{2}+p_{3}\right)^{2},
\end{array}
$$

supplemented by the auxiliary denominators

$$
\mathcal{D}_{8}=\left(k_{1}+p_{2}\right)^{2}, \quad \mathcal{D}_{9}=\left(k_{2}+p_{1}+p_{2}+p_{3}\right)^{2} .
$$

For the three-loop ladder diagram in figure $1 \mathrm{~b}$ one has instead

$$
\begin{array}{llr}
\mathcal{D}_{1}=k_{1}^{2}, \quad \mathcal{D}_{2}=k_{2}^{2}, & \mathcal{D}_{3}=k_{3}^{2}, & \mathcal{D}_{4}=\left(k_{1}-k_{2}\right)^{2}, \\
\mathcal{D}_{5}=\left(k_{2}-k_{3}\right)^{2}, & \mathcal{D}_{6}=\left(k_{3}+p_{2}\right)^{2}, & \mathcal{D}_{7}=\left(k_{1}+p_{2}+p_{3}\right)^{2}, \\
\mathcal{D}_{8}=\left(k_{2}+p_{2}+p_{3}\right)^{2}, & \mathcal{D}_{9}=\left(k_{3}+p_{2}+p_{3}\right)^{2}, & \mathcal{D}_{10}=\left(k_{1}+p_{1}+p_{2}+p_{3}\right)^{2},
\end{array}
$$

supplemented by the auxiliary denominators

$$
\begin{array}{ll}
\mathcal{D}_{11}=\left(k_{1}+p_{2}\right)^{2}, \quad \mathcal{D}_{12}=\left(k_{2}+p_{2}\right)^{2}, & \mathcal{D}_{13}=\left(k_{2}+p_{1}+p_{2}+p_{3}\right)^{2}, \\
\mathcal{D}_{14}=\left(k_{3}+p_{1}+p_{2}+p_{3}\right)^{2}, & \mathcal{D}_{15}=\left(k_{3}-k_{1}\right)^{2} .
\end{array}
$$

In the following we consider $\ell$-loop Feynman integrals built out of $p$ of the above denominators, each raised to some integer power, of the form

$$
\int \widetilde{\mathrm{d}^{d} k_{1}} \ldots \widetilde{\mathrm{d}^{d} k_{\ell}} \frac{1}{\mathcal{D}_{a_{1}}^{n_{1}} \ldots \mathcal{D}_{a_{p}}^{n_{p}}}
$$

where the integration measure is defined as

$$
\widetilde{\mathrm{d}^{d} k_{i}} \equiv \frac{\mathrm{d}^{d} k_{i}}{(2 \pi)^{d}}\left(\frac{i S_{\epsilon}}{16 \pi^{2}}\right)^{-1}\left(-m^{2}\right)^{\epsilon}
$$

with

$$
S_{\epsilon} \equiv(4 \pi)^{\epsilon} \frac{\Gamma(1+\epsilon) \Gamma^{2}(1-\epsilon)}{\Gamma(1-2 \epsilon)} .
$$

The two- and three-loop MI's, respectively depicted in figure 2 and figures. $3-5$, are functions of the kinematic variables

$$
s=\left(p_{2}+p_{3}\right)^{2}, \quad t=\left(p_{1}+p_{3}\right)^{2}, \quad u=\left(p_{1}+p_{2}\right)^{2}, \quad m^{2}=\left(p_{1}+p_{2}+p_{3}\right)^{2},
$$


with $p_{i}^{2}=0(i=1,2,3)$ and $p_{4}^{2}=\left(p_{1}+p_{2}+p_{3}\right)^{2}$, fulfilling $s+t+u=m^{2}$. For convenience, we define the dimensionless ratios

$$
x \equiv \frac{s}{m^{2}}, \quad y \equiv \frac{t}{m^{2}}, \quad z \equiv \frac{u}{m^{2}}, \quad \text { with } x+y+z=1 .
$$

For planar topologies, like the ones we consider, one can always choose the Euclidean kinematic region $m^{2}, s, t, u<0$ such that the MI's are real. For definiteness, we work in the region $0<y<1,0<x<1-y$ (or equivalently $0<x<1,0<y<1-x$ ). The analytic continuation of our results to regions of physical interests can be performed by generalizing to higher weights the procedure of ref. [65].

The sets of MI's we choose to work with (see sections 3 and 4) obey $\epsilon$-linear systems of first order differential equations in the kinematic variables $(\sigma=x, y)$,

$$
\partial_{\sigma} f\left(\epsilon, m^{2}, x, y\right)=A_{\sigma}\left(\epsilon, m^{2}, x, y\right) f\left(\epsilon, m^{2}, x, y\right)
$$

with

$$
A_{\sigma}\left(\epsilon, m^{2}, x, y\right)=A_{\sigma, 0}\left(m^{2}, x, y\right)+\epsilon A_{\sigma, 1}\left(m^{2}, x, y\right) .
$$

Given that $m^{2}$ is the only dimensionful variable, the differential equation in $m^{2}$ is related to the scaling equation. A factor $\left(-m^{2}\right)^{-\ell \epsilon}$ is already included in integration measure (2.7), therefore for each $f_{i}$ we simply have

$$
\partial_{m^{2}} f_{i}\left(m^{2}, x, y, \epsilon\right)=-\frac{n_{i}}{m^{2}} f_{i}\left(\epsilon, m^{2}, x, y\right),
$$

where $n_{i}$ is the dimension of the integral $f_{i}$ in units of a squared mass.

\subsection{Magnus exponential}

We can use Magnus exponential $[31,38]$ to define a matrix $B$ that implements a change of basis $f \rightarrow g$,

$$
f \equiv B g, \quad \hat{A}_{\sigma} \equiv B^{-1} A_{\sigma} B-B^{-1} \partial_{\sigma} B
$$

such that the new basis of MI's fulfills a canonical system of differential equations,

$$
\partial_{\sigma} g(\epsilon, x, y)=\epsilon \hat{A}_{\sigma}(x, y) g(\epsilon, x, y)
$$

where the dependence on $\epsilon$ is factorized from that on the kinematic variables [24]. Accordingly, $g$ is called canonical basis of MI's.

Following ref. [31], in order to build the matrix $B$ of (2.13), let us introduce Magnus exponential matrix $[38,39]$. For a generic matrix $M=M(t)$, this is defined as

$$
e^{\Omega[M(t)]},
$$

being the solution of the following matrix differential equation,

$$
\partial_{t} e^{\Omega[M(t)]}=M(t) e^{\Omega[M(t)]} .
$$


The exponent $\Omega[M(t)]$ is given as a series of matrices obtained by repeated integrations, using $M(t)$ as a kernel,

$$
\Omega[M(t)] \equiv \sum_{n=1}^{\infty} \Omega_{n}[M(t)],
$$

where the first three terms of the series expansion read,

$$
\begin{aligned}
& \Omega_{1}[M(t)]=\int_{t_{0}}^{t} d \tau_{1} M\left(\tau_{1}\right), \\
& \Omega_{2}[M(t)]=\frac{1}{2} \int_{t_{0}}^{t} d \tau_{1} \int_{t_{0}}^{\tau_{1}} d \tau_{2}\left[M\left(\tau_{1}\right), M\left(\tau_{2}\right)\right], \\
& \Omega_{3}[M(t)]=\frac{1}{6} \int_{t_{0}}^{t} d \tau_{1} \int_{t_{0}}^{\tau_{1}} d \tau_{2} \int_{t_{0}}^{\tau_{2}} d \tau_{3}\left[M\left(\tau_{1}\right),\left[M\left(\tau_{2}\right), M\left(\tau_{3}\right)\right]\right]+\left[M\left(\tau_{3}\right),\left[M\left(\tau_{2}\right), M\left(\tau_{1}\right)\right]\right] .
\end{aligned}
$$

\subsection{Canonical transformation}

The basis change $B$, which brings the systems in the canonical form (2.14), can in general be obtained in both the two- and three-loop cases as the product of five exponential matrices, each implementing a change of basis according to (2.13), applied in successions. The dependence of each matrix on $x$ and $y$ is understood.

1. First of all we rescale all the MI's by appropriate factors of $m^{2}$ in order to work with dimensionless integrals. This can be conveniently implemented by a Magnus exponential of the diagonal matrix $A_{m^{2}, 0}$

$$
A_{\sigma} \rightarrow A_{\sigma}^{[0]}, \quad B^{[0]} \equiv e^{\Omega\left[A_{m^{2}, 0}\right]} .
$$

As a result of this transformation, $A_{m^{2}}^{[0]}$ vanishes and the MI's do not depend anymore on $m^{2}$.

2. Then we decompose $A_{x, 0}^{[0]}$ in a diagonal term $D_{x, 0}^{[0]}$ and a off-diagonal one $N_{x, 0}^{[0]}$,

$$
A_{x, 0}^{[0]}=D_{x, 0}^{[0]}+N_{x, 0}^{[0]},
$$

and use only the diagonal part for the next basis change,

$$
A_{\sigma}^{[0]} \rightarrow A_{\sigma}^{[1]}, \quad B^{[1]} \equiv e^{\Omega\left[D_{x, 0}^{[0]}\right]} .
$$

3. We repeat as before and split $A_{y, 0}^{[1]}$ into its diagonal and off-diagonal parts,

$$
A_{y, 0}^{[1]}=D_{y, 0}^{[1]}+N_{y, 0}^{[1]}
$$

and we build the Magnus exponential again using the diagonal part

$$
A_{\sigma}^{[1]} \rightarrow A_{\sigma}^{[2]}, \quad B^{[2]} \equiv e^{\Omega\left[D_{y, 0}^{[0]}\right]} .
$$


4. Now the matrix $A_{x, 0}^{[2]}$, has no diagonal term left,

$$
A_{x, 0}^{[2]}=N_{x, 0}^{[2]},
$$

therefore we build the Magnus exponential of the off-diagonal part

$$
A_{\sigma}^{[2]} \rightarrow A_{\sigma}^{[3]}, \quad B^{[3]} \equiv e^{\Omega\left[N_{x, 0}^{[2]}\right]} .
$$

5. The matrix $A_{y, 0}^{[3]}$ has no diagonal term as well,

$$
A_{y, 0}^{[3]}=N_{y, 0}^{[3]},
$$

so we can define the last basis change

$$
A_{\sigma}^{[3]} \rightarrow A_{\sigma}^{[4]}, \quad B^{[4]} \equiv e^{\Omega\left[N_{y, 0}^{[3]}\right]} .
$$

After the last transformation we observe that

$$
A_{x, 0}^{[4]}=0=A_{y, 0}^{[4]} .
$$

This means that the basis change of (2.13), with the matrix $B$ given by

$$
\left.B \equiv B^{[0]} B^{[1]} B^{[2]} B^{[3]} B^{[4]}=e^{\Omega\left[A_{m}^{2}, 0\right.}\right] e^{\Omega\left[D_{x, 0}^{[0]}\right]} e^{\Omega\left[D_{y, 0}^{[1]}\right]} e^{\Omega\left[N_{x, 0}^{[2]}\right]} e^{\Omega\left[N_{y, 0}^{[3]}\right]},
$$

absorbs the constant terms of $A_{x}$ and $A_{y}$ in the $\epsilon$-linear systems in (2.10) and brings them to the canonical form (2.14):

$$
A_{\sigma}\left(\epsilon, m^{2}, x, y\right) \rightarrow \epsilon \hat{A}_{\sigma}(x, y)
$$

\subsection{Canonical system}

The two partial-derivative systems satisfied by the new set of MI's, $g=B^{-1} f$, can be conveniently combined in an exact differential form,

$$
d g(\epsilon, x, y)=\epsilon d \hat{\mathcal{A}}(x, y) g(\epsilon, x, y), \quad d \hat{\mathcal{A}} \equiv \hat{A}_{x} d x+\hat{A}_{y} d y
$$

where $\hat{\mathcal{A}}(x, y)$ is logarithmic in the variables $x$ and $y$,

$$
\begin{aligned}
\hat{\mathcal{A}}(x, y)= & M_{1} \log (x)+M_{2} \log (1-x)+M_{3} \log (y)+M_{4} \log (1-y)+ \\
& +M_{5} \log \left(\frac{x+y}{x}\right)+M_{6} \log \left(\frac{1-x-y}{1-x}\right) .
\end{aligned}
$$

The matrices $M_{i}$ are $n \times n$ sparse matrices with purely rational entries, where $n$ is the number of MI's. The value of $n$ depends on the number of loops, and it amounts to $n=4,18,85$, respectively at one-, two- and three-loop. The arguments of the logarithms are defined as letters, and the set of letters

$$
\{x, 1-x, y, 1-y, x+y, 1-x-y\}
$$


constitutes the alphabet. We observe that this alphabet is common both to the two- and the three loop cases. At one-loop [18], although not shown here, $M_{5}$ is absent, while at two-loop $M_{5}$ has only one non-vanishing entry.

The solution of (2.14), (2.31) can be expressed as a Dyson series in $\epsilon$,

$$
g(x, y, \epsilon)=\left(1+\sum_{n=1}^{\infty} \epsilon^{n} D^{(n)}(x, y)\right) g_{0}(\epsilon),
$$

where the (matrix) coefficients of the series can be written as the iterated line integral,

$$
D^{(n)}(x, y) \equiv \int_{\gamma} d \hat{\mathcal{A}}_{1} d \hat{\mathcal{A}}_{2} \cdots d \hat{\mathcal{A}}_{n}
$$

where $d \hat{\mathcal{A}}_{i} \equiv d \hat{\mathcal{A}}\left(x_{i}, y_{i}\right)$. Equivalently, the solution admits a representation in terms of the Magnus exponential

$$
g(x, y, \epsilon)=e^{\Omega[\epsilon d \hat{\mathcal{A}}](x, y)} g_{0}(\epsilon)
$$

where the vector $g_{0}(\epsilon) \equiv g\left(x_{0}, y_{0}, \epsilon\right)$ corresponds to the boundary values of the MI's. This form is very suggestive, as Magnus exponential can be considered as an evolution operator, like in the unitary formalism, that brings the MI's $g$ from their initial, boundary values to the considered point in the $(x, y)$-plane. ${ }^{1}$

For definiteness, we integrate the exact differential form (2.31) from an arbitrary point $\left(x_{0}, y_{0}\right)$ to $(x, y)$, along the broken path composed of the two segments in which one of the variables is kept constant. The integration is performed order by order in $\epsilon$, up to a multiplicative vector of unknown constants. The latter are fixed by requiring the regularity of $g(x, y, \epsilon)$ at the pseudothresholds (see section 5).

The solution of the canonical system in (2.32) with the coefficient matrix given by (2.31) can be naturally expressed in terms of Goncharov's multiple polylogarithms ( $G$-polylogarithms, for short) [26, 41-43],

$$
\begin{aligned}
G\left(\vec{w}_{n} ; x\right) & \equiv G\left(w_{1}, \vec{w}_{n-1} ; x\right) \equiv \int_{0}^{x} d t \frac{1}{t-w_{1}} G\left(\vec{w}_{n-1} ; t\right), \\
G\left(\overrightarrow{0}_{n} ; x\right) & \equiv \frac{1}{n !} \log ^{n}(x)
\end{aligned}
$$

with $\vec{w}_{n}$ being a vector of $n$ arguments. The number $n$ is referred to as the weight of $G\left(\vec{w}_{n} ; x\right)$ and amounts to the number of iterated integrations needed to define it. Equivalently one has

$$
\partial_{x} G\left(\vec{w}_{n} ; x\right)=\partial_{x} G\left(w_{1}, \vec{w}_{n-1} ; x\right)=\frac{1}{x-w_{1}} G\left(\vec{w}_{n-1} ; x\right) .
$$

\footnotetext{
${ }^{1}$ In this case, the evolution has to be understood like the variation w.r.t. the kinematic invariants that are the variables of the system of differential equations obeyed by MI's, rather than the quantum-mechanical time-evolution. Moreover, the matrices representing the systems of differential equations for MI's are not unitary.
} 


\begin{tabular}{|c|rrr|}
\hline weight & $\alpha=2$ & $\alpha=3$ & $\alpha=4$ \\
\hline 1 & 2 & 3 & 4 \\
2 & 1 & 3 & 6 \\
3 & 2 & 8 & 20 \\
4 & 3 & 18 & 60 \\
5 & 6 & 48 & 204 \\
6 & 9 & 116 & 670 \\
\hline
\end{tabular}

Table 1. Size of a basis of $G$-polylogarithms.

$G$-polylogarithms fulfill shuffle algebra relations of the type

$$
G(\vec{m} ; x) G(\vec{n} ; x)=G(\vec{m} ; x) \sqcup G(\vec{n} ; x)=\sum_{\vec{p}=\vec{m} \sqcup \vec{n}} G(\vec{p} ; x),
$$

where shuffle product $\vec{m} \sqcup \vec{n}$ denotes all possible merges of $\vec{m}$ and $\vec{n}$ preserving their respective orderings. Because of shuffle relations, for a given alphabet and a given weight one can identify a minimal basis of $G$-polylogarithms. For the calculation of the considered two- and three-loop MI's the alphabet in (2.33) corresponds to the set of weights $W=$ $\{0,1,-x, 1-x\}$. The number of basis elements depending on the weight $n$ and the alphabet size $\alpha$ is given by the Witt formula:

$$
N(n, \alpha)=\frac{1}{n} \sum_{d \mid n} \mu(d) \alpha^{\frac{n}{d}},
$$

where $\mu$ denotes the Möbius function and the sum is done over all divisors of $n$. For reference we give values of $N(n, \alpha)$ relevant for this calculation in table 1 .

\section{Two-loop master integrals}

A set of 18 two-loop MI's for the planar massless box integrals with one massive leg of figure 1a was first computed in refs. $[18,59]$. We present the calculation of an alternative, yet compatible set of MI's. We begin by choosing the following basis

$$
\begin{aligned}
& f_{1}=\epsilon^{2} \mathcal{T}_{1} \\
& f_{2}=\epsilon^{2} \mathcal{T}_{2} \\
& f_{3}=\epsilon^{2} \mathcal{T}_{3} \\
& f_{4}=\epsilon^{2} \mathcal{T}_{4} \\
& f_{5}=\epsilon^{3} \mathcal{T}_{5} \\
& f_{6}=\epsilon^{2} \mathcal{T}_{6} \\
& f_{7}=\epsilon^{3} \mathcal{T}_{7} \\
& f_{8}=\epsilon^{3} \mathcal{T}_{8} \\
& f_{9}=\epsilon^{3} \mathcal{T}_{9} \\
& f_{10}=\epsilon^{4} \mathcal{T}_{10} \\
& f_{11}=\epsilon^{3} \mathcal{T}_{11} \\
& f_{12}=\epsilon^{4} \mathcal{T}_{12} \\
& f_{13}=\epsilon^{4} \mathcal{T}_{13} \\
& f_{14}=\epsilon^{3} \mathcal{T}_{14} \\
& f_{15}=\epsilon^{3} \mathcal{T}_{15} \\
& f_{16}=\epsilon^{4} \mathcal{T}_{16} \\
& f_{17}=\epsilon^{4} \mathcal{T}_{17} \\
& f_{18}=\epsilon^{4} \mathcal{T}_{18}
\end{aligned}
$$

where the integrals $\mathcal{T}_{i}$ are depicted in figure 2 . The set $\{f\}_{i=1, \ldots, 18}$ is chosen to obey a $\epsilon$-linear system of differential equations in $x$ and $y$, which, as previously described, can be 


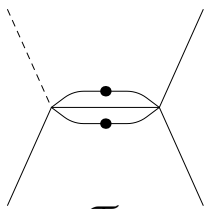

$\mathcal{T}_{1}$

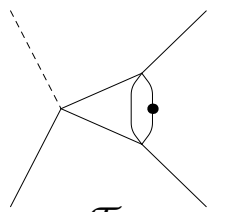

$\mathcal{T}_{5}$

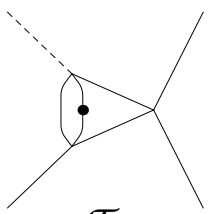

$\mathcal{T}_{9}$

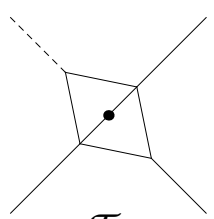

$\mathcal{T}_{14}$

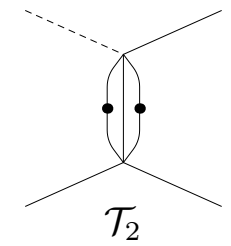

$\mathcal{T}_{2}$

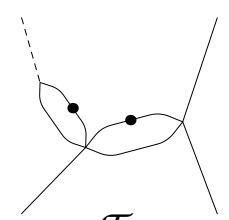

$\mathcal{T}_{6}$

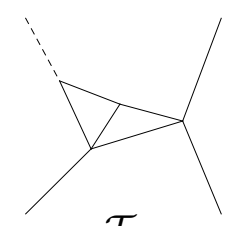

$\mathcal{T}_{10}$

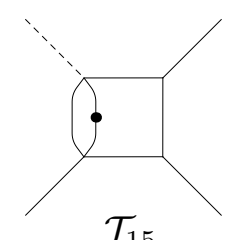

$\mathcal{T}_{15}$
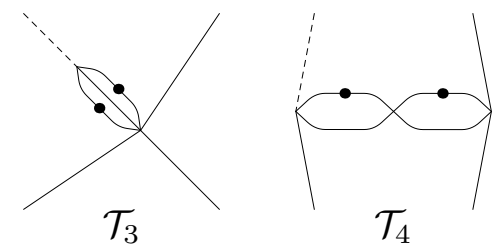

$\mathcal{T}_{4}$

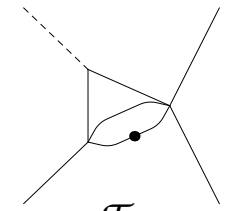

$\mathcal{T}_{7}$
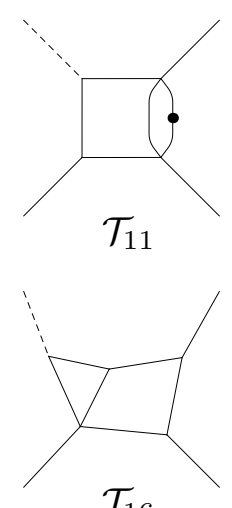

$\mathcal{T}_{16}$
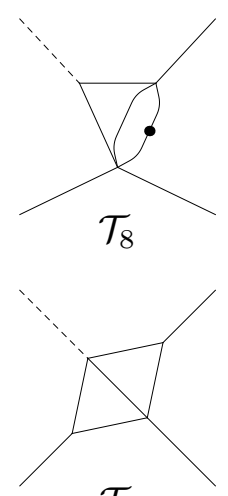

$\mathcal{T}_{12}$
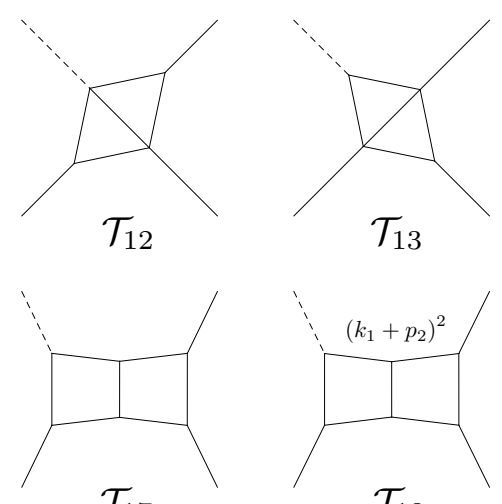

$\mathcal{T}_{17}$

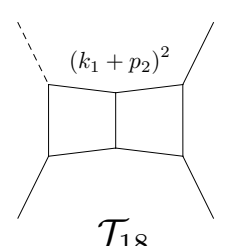

$\mathcal{T}_{18}$

Figure 2. Two-loop Master Integrals $\left\{\mathcal{T}_{i}\right\}_{i=1, \ldots, 18}$. The solid lines stand for massless particles; the dashed line represents a massive particle; dots indicate squared propagators; numerators may appear as indicated $\left(p_{i j} \equiv p_{i}+p_{j}\right)$.

cast in canonical form by means of Magnus exponentials. In this case, the canonical transformation $B$, generically defined in (2.29), reduces to $B \equiv e^{\Omega\left[A_{m^{2}, 0}\right]} e^{\Omega\left[D_{x, 0}^{[0]}\right]} e^{\Omega\left[D_{y, 0}^{[1]}\right]}$, because after the first three transformations $N_{x, 0}^{[2]}=N_{y, 0}^{[2]}=0$. The canonical basis $\{g\}_{i=1, \ldots, 18}$ reads,

$$
\begin{aligned}
g_{1} & =s f_{1} \\
g_{4} & =s^{2} f_{4} \\
g_{7} & =\lambda_{s} f_{7} \\
g_{10} & =\lambda_{s} f_{10} \\
g_{13} & =\lambda_{u} f_{13} \\
g_{16} & =s \lambda_{t} f_{16}
\end{aligned}
$$$$
g_{2}=t f_{2}
$$$$
g_{3}=m^{2} f_{3}
$$$$
g_{5}=s f_{5}
$$$$
g_{6}=m^{2} s f_{6}
$$$$
g_{8}=\lambda_{t} f_{8}
$$$$
g_{9}=\lambda_{s} f_{9}
$$$$
g_{11}=s t f_{11}
$$$$
g_{12}=u f_{12}
$$$$
g_{14}=s t f_{14}
$$$$
g_{15}=s t f_{15}
$$$$
g_{17}=s^{2} t f_{17}
$$

where $\lambda_{a}=\left(m^{2}-a\right)$. The sparse matrices $M_{i}(i=1, \ldots, 6)$ appearing in the corresponding canonical system in (2.31) and (2.32) are given in appendix A. 


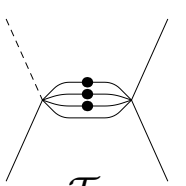

$\mathcal{T}_{1}$

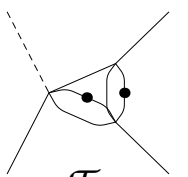

$\mathcal{T}_{7}$

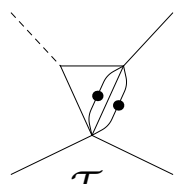

$\mathcal{T}_{13}$

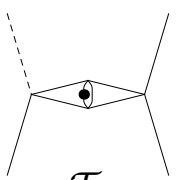

$\mathcal{T}_{19}$

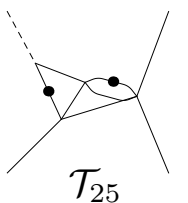

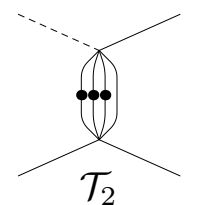

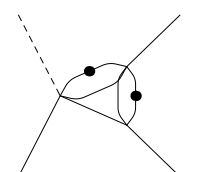

$\mathcal{T}_{8}$

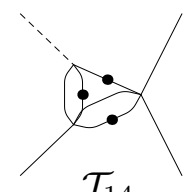

$\mathcal{T}_{14}$
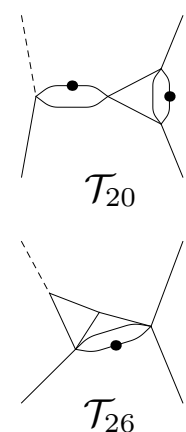

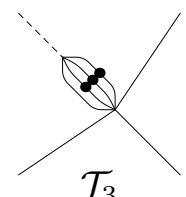

$\mathcal{T}_{3}$

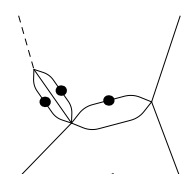

$\mathcal{T}_{9}$

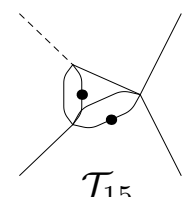

$\mathcal{T}_{15}$

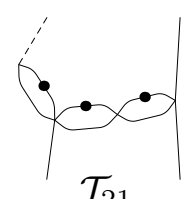

$\mathcal{T}_{21}$

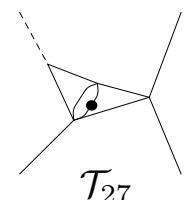

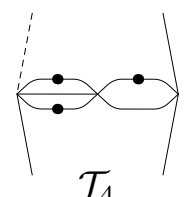

$\mathcal{T}_{4}$

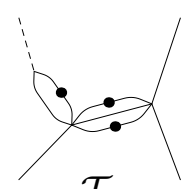

$\mathcal{T}_{10}$

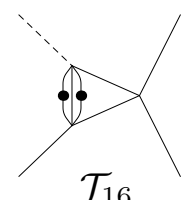

$\mathcal{T}_{16}$

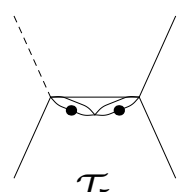

$\mathcal{T}_{5}$

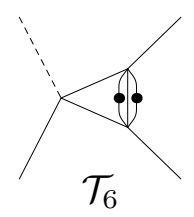

$\mathcal{T}_{6}$

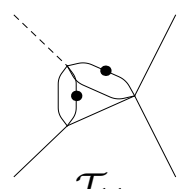

$\mathcal{T}_{11}$

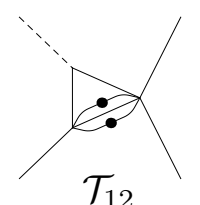

$\mathcal{T}_{12}$
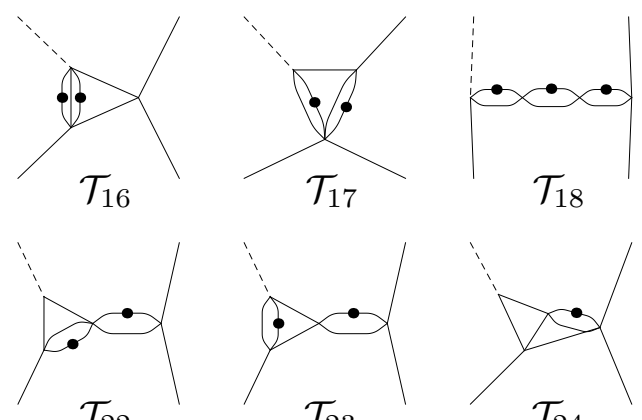

$\mathcal{T}_{22}$

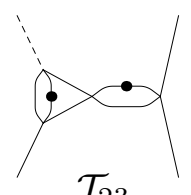

$\mathcal{T}_{23}$

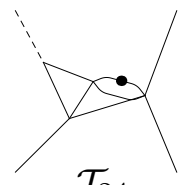

$\mathcal{T}_{24}$

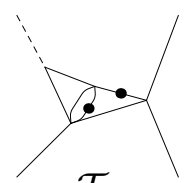

$\mathcal{T}_{28}$

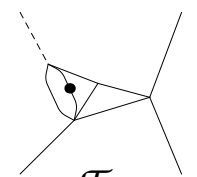

$\mathcal{T}_{29}$

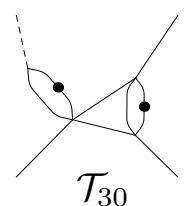

Figure 3. Three-loop Master Integrals $\left\{\mathcal{T}_{i}\right\}_{i=1, \ldots, 30}$. The solid lines stand for massless particles; the dashed line represents a massive particle; dots indicate squared propagators; numerators may appear as indicated $\left(p_{i j} \equiv p_{i}+p_{j}\right)$. See also figures 4 and 5 .

\section{Three-loop master integrals}

The calculation of the three-loop ladder box integrals with one massive leg, represented in figure $1 \mathrm{~b}$, is the main, new result of this paper. After performing the automatic reduction by means of the computer code Reduze 2 [60,61], we identify a set of 85 MI's,
$f_{1}=\epsilon^{3} \mathcal{T}_{1}$
$f_{2}=\epsilon^{3} \mathcal{T}_{2}$
$f_{3}=\epsilon^{3} \mathcal{T}_{3}$
$f_{4}=\epsilon^{3} \mathcal{T}_{4}$
$f_{5}=\epsilon^{3}(1+2 \epsilon) \mathcal{T}_{5}$
$f_{6}=\epsilon^{4} \mathcal{T}_{6}$
$f_{7}=\epsilon^{4} \mathcal{T}_{7}$
$f_{8}=\epsilon^{4} \mathcal{T}_{8}$
$f_{9}=\epsilon^{3} \mathcal{T}_{9}$
$f_{10}=\epsilon^{3} \mathcal{T}_{10}$
$f_{11}=\epsilon^{4} \mathcal{T}_{11}$
$f_{12}=\epsilon^{4} \mathcal{T}_{12}$
$f_{13}=\epsilon^{4} \mathcal{T}_{13}$
$f_{14}=\epsilon^{3} \mathcal{T}_{14}$
$f_{15}=\epsilon^{4} \mathcal{T}_{15}$
$f_{16}=\epsilon^{4} \mathcal{T}_{16}$
$f_{17}=\epsilon^{4} \mathcal{T}_{17}$
$f_{18}=\epsilon^{3} \mathcal{T}_{18}$
$f_{19}=\epsilon^{4}(1-2 \epsilon) \mathcal{T}_{19}$
$f_{20}=\epsilon^{4} \mathcal{T}_{20}$
$f_{21}=\epsilon^{3} \mathcal{T}_{21}$
$f_{22}=\epsilon^{4} \mathcal{T}_{22}$
$f_{23}=\epsilon^{4} \mathcal{T}_{23}$
$f_{24}=\epsilon^{5} \mathcal{T}_{24}$
$f_{25}=\epsilon^{4} \mathcal{T}_{25}$
$f_{26}=\epsilon^{5} \mathcal{T}_{26}$
$f_{27}=\epsilon^{5} \mathcal{T}_{27}$ 


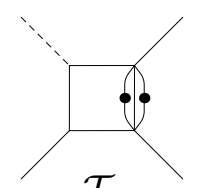

$\mathcal{T}_{31}$

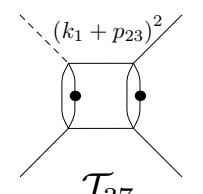

$\mathcal{T}_{37}$

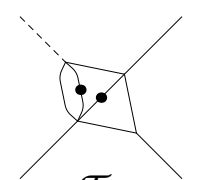

$\mathcal{T}_{43}$

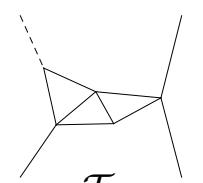

$\mathcal{T}_{49}$

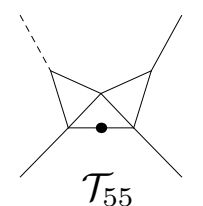

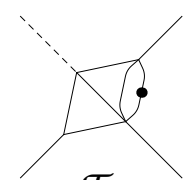

$\mathcal{T}_{32}$

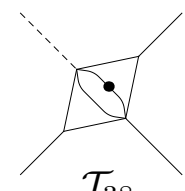

$\mathcal{T}_{38}$

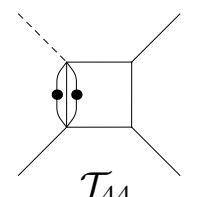

$\mathcal{T}_{44}$

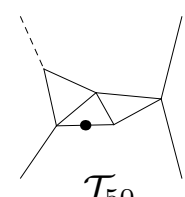

$\mathcal{T}_{50}$

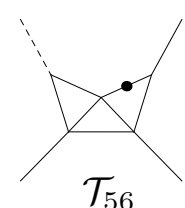

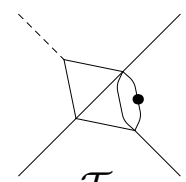

$\mathcal{T}_{33}$

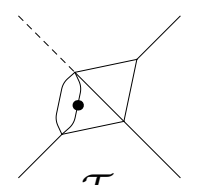

$\mathcal{T}_{39}$

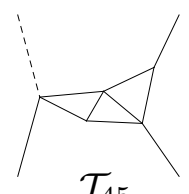

$\mathcal{T}_{45}$

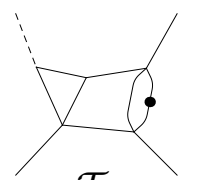

$\mathcal{T}_{51}$

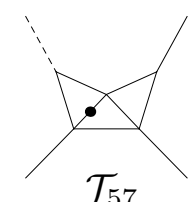

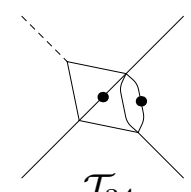

$\mathcal{T}_{34}$

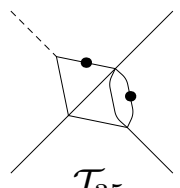

$\mathcal{T}_{35}$
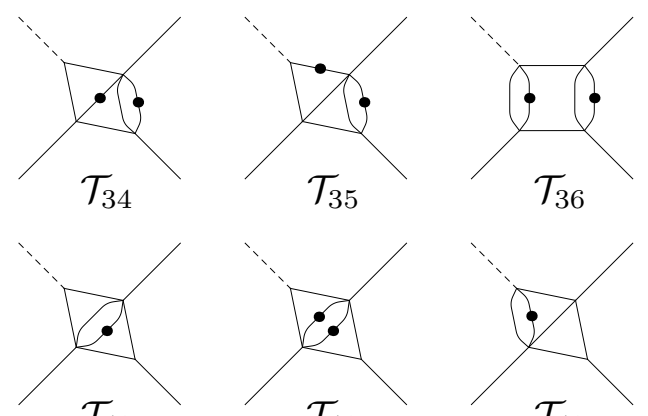

$\mathcal{T}_{40}$

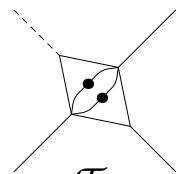

$\mathcal{T}_{41}$
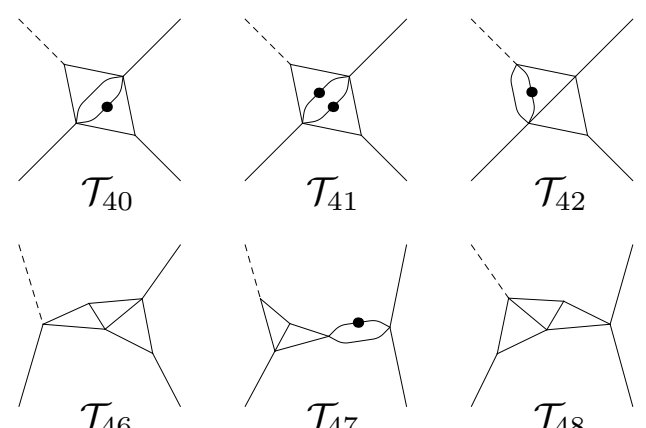

$\mathcal{T}_{46}$

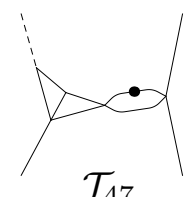

$\mathcal{T}_{47}$
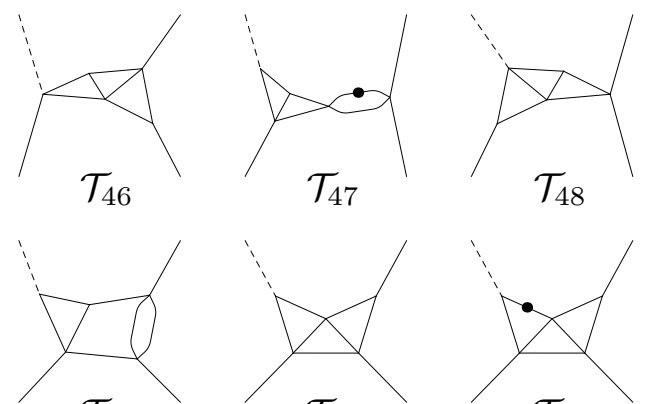

$\mathcal{T}_{52}$

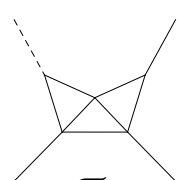

$\mathcal{T}_{53}$
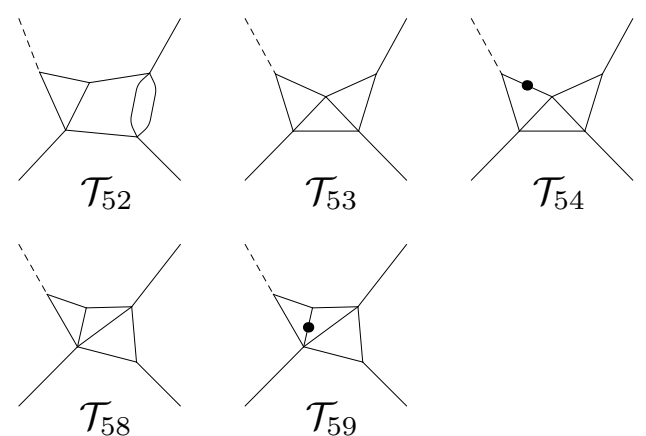

Figure 4. Three-loop Master Integrals $\left\{\mathcal{T}_{i}\right\}_{i=31, \ldots, 59}$. The solid lines stand for massless particles; the dashed line represents a massive particle; dots indicate squared propagators; numerators may appear as indicated $\left(p_{i j} \equiv p_{i}+p_{j}\right)$. See also figures 3 and 5 .

\begin{tabular}{|c|c|c|}
\hline$f_{28}=\epsilon^{3}(1+2 \epsilon) \mathcal{T}_{28}$ & $f_{29}=\epsilon^{5} \mathcal{T}_{29}$ & $f_{30}=\epsilon^{4} \mathcal{T}_{30}$ \\
\hline$f_{31}=\epsilon^{4} \mathcal{T}_{31}$ & $f_{32}=\epsilon^{5} \mathcal{T}_{32}$ & $f_{33}=\epsilon^{5} \mathcal{T}_{33}$ \\
\hline$f_{34}=\epsilon^{4} \mathcal{T}_{34}$ & $f_{35}=\epsilon^{4} \mathcal{T}_{35}$ & $f_{36}=\epsilon^{4} \mathcal{T}_{36}$ \\
\hline$f_{37}=\epsilon^{4} \frac{(1-2 \epsilon)}{1-\epsilon} \mathcal{T}_{37}$ & $f_{38}=\epsilon^{5} \mathcal{T}_{38}$ & $f_{39}=\epsilon^{5} \mathcal{T}_{39}$ \\
\hline$f_{40}=\epsilon^{5} \mathcal{T}_{40}$ & $f_{41}=\epsilon^{4} \mathcal{T}_{41}$ & $f_{42}=\epsilon^{5} \mathcal{T}_{42}$ \\
\hline$f_{43}=\epsilon^{4} \mathcal{T}_{43}$ & $f_{44}=\epsilon^{4} \mathcal{T}_{44}$ & $f_{45}=\epsilon^{6} \mathcal{T}_{45}$ \\
\hline$f_{46}=\epsilon^{6} \mathcal{T}_{46}$ & $f_{47}=\epsilon^{5} \mathcal{T}_{47}$ & $f_{48}=\epsilon^{6} \mathcal{T}_{48}$ \\
\hline$f_{49}=\epsilon^{6} \mathcal{T}_{49}$ & $f_{50}=\epsilon^{4}(1+\epsilon) \mathcal{T}_{50}$ & $f_{51}=\epsilon^{5} \mathcal{T}_{51}$ \\
\hline$f_{52}=\epsilon^{5}(1-2 \epsilon) \mathcal{T}_{52}$ & $f_{53}=\epsilon^{6} \mathcal{T}_{53}$ & $f_{54}=\epsilon^{5} \mathcal{T}_{54}$ \\
\hline$f_{55}=\epsilon^{5} \mathcal{T}_{55}$ & $f_{56}=\epsilon^{4}(1+\epsilon) \mathcal{T}_{56}$ & $f_{57}=\epsilon^{5} \mathcal{T}_{57}$ \\
\hline$f_{58}=\epsilon^{6} \mathcal{T}_{58}$ & $f_{59}=\epsilon^{4}(1+\epsilon) \mathcal{T}_{59}$ & $f_{60}=\epsilon^{6} \mathcal{T}_{60}$ \\
\hline$f_{61}=\epsilon^{5} \mathcal{T}_{61}$ & $f_{62}=\epsilon^{5} \mathcal{T}_{62}$ & $f_{63}=\epsilon^{5} \mathcal{T}_{63}$ \\
\hline
\end{tabular}




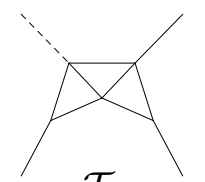

$\mathcal{T}_{60}$

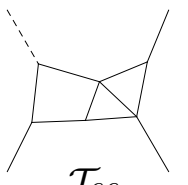

$\mathcal{T}_{66}$

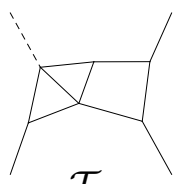

$\mathcal{T}_{72}$

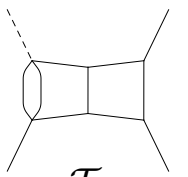

$\mathcal{T}_{78}$

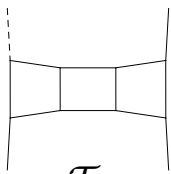

$\mathcal{T}_{83}$

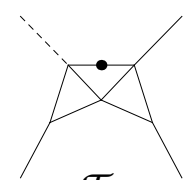

$\mathcal{T}_{61}$

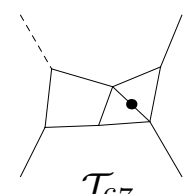

$\mathcal{T}_{67}$

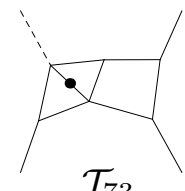

$\mathcal{T}_{73}$

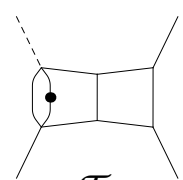

$\mathcal{T}_{79}$

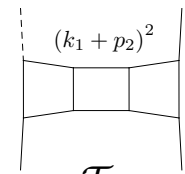

$\mathcal{T}_{84}$

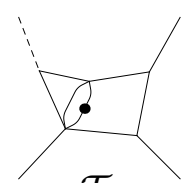

$\mathcal{T}_{62}$

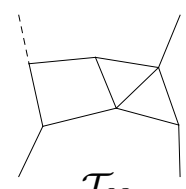

$\mathcal{T}_{68}$

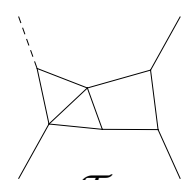

$\mathcal{T}_{74}$

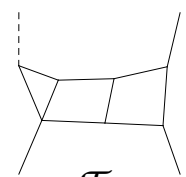

$\mathcal{T}_{80}$

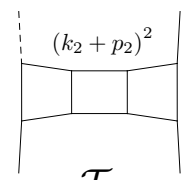

$\mathcal{T}_{85}$

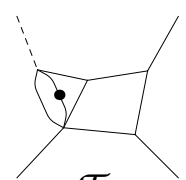

$\mathcal{T}_{63}$

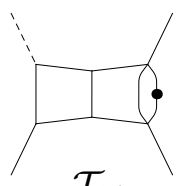

$\mathcal{T}_{64}$
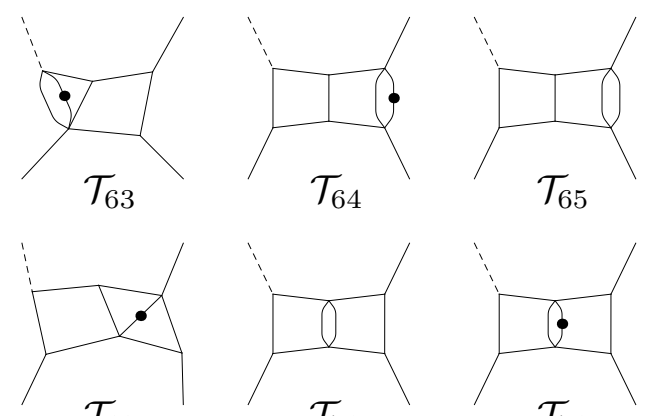

$\mathcal{T}_{69}$

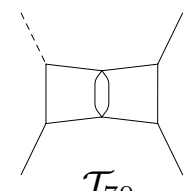

$\mathcal{T}_{70}$
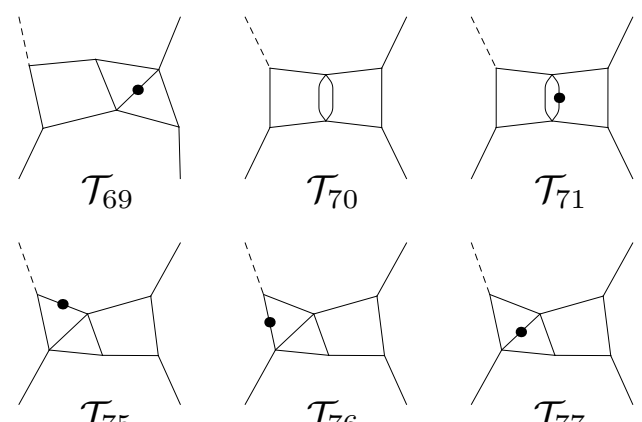

$\mathcal{T}_{75}$
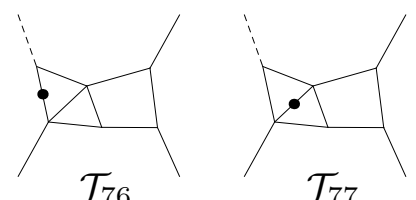

$\mathcal{T}_{77}$

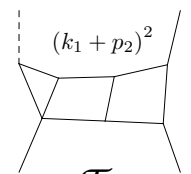

$\mathcal{T}_{81}$

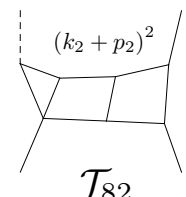

$\mathcal{T}_{82}$

Figure 5. Three-loop Master Integrals $\left\{\mathcal{T}_{i}\right\}_{i=60, \ldots, 85}$. The solid lines stand for massless particles; the dashed line represents a massive particle; dots indicate squared propagators; numerators may appear as indicated $\left(p_{i j} \equiv p_{i}+p_{j}\right)$. See also figures 3 and 4 .

$$
\begin{aligned}
& f_{64}=\epsilon^{5} \mathcal{T}_{64} \\
& f_{65}=\epsilon^{5}(1-2 \epsilon) \mathcal{T}_{65} \quad f_{66}=\epsilon^{6} \mathcal{T}_{66} \\
& f_{67}=\epsilon^{5} \mathcal{T}_{67} \\
& f_{68}=\epsilon^{6} \mathcal{T}_{68} \\
& f_{69}=\epsilon^{5} \mathcal{T}_{69} \\
& f_{70}=\epsilon^{5}(1-2 \epsilon) \mathcal{T}_{70} \\
& f_{71}=\epsilon^{5} \mathcal{T}_{71} \\
& f_{72}=\epsilon^{6} \mathcal{T}_{72} \\
& f_{73}=\epsilon^{5} \mathcal{T}_{73} \\
& f_{74}=\epsilon^{6} \mathcal{T}_{74} \\
& f_{75}=\epsilon^{5} \mathcal{T}_{75} \\
& f_{76}=\epsilon^{5} \mathcal{T}_{76} \\
& f_{77}=\epsilon^{5} \mathcal{T}_{77} \\
& f_{78}=\epsilon^{5}(1-2 \epsilon) \mathcal{T}_{78} \\
& f_{79}=\epsilon^{5} \mathcal{T}_{79} \\
& f_{80}=\epsilon^{6} \mathcal{T}_{80} \\
& f_{81}=\epsilon^{5}(1-2 \epsilon) \mathcal{T}_{81} \\
& f_{82}=\epsilon^{6} \mathcal{T}_{82} \\
& f_{83}=\epsilon^{6} \mathcal{T}_{83} \\
& f_{84}=\epsilon^{6} \mathcal{T}_{84} \\
& f_{85}=\epsilon^{6} \mathcal{T}_{85}
\end{aligned}
$$

where the integrals $\mathcal{T}_{i}$ are depicted in figures $3-5$. As before, the choice of $\{f\}_{i=1, \ldots, 85}$ is motivated by them obeying a $\epsilon$-linear system of differential equations in $x$ and $y$.

In this case, the canonical transformation $B$, generically defined in (2.29), reduces to $B \equiv e^{\Omega\left[A_{m^{2}, 0}\right]} e^{\Omega\left[D_{x, 0}^{[0]}\right]} e^{\Omega\left[D_{y, 0}^{[1]}\right]} e^{\Omega\left[N_{x, 0}^{[2]}\right]}$, yielding the canonical basis $\{g\}_{i=1, \ldots, 85}$

$$
\begin{array}{llll}
g_{1}=s f_{1} & g_{2}=t f_{2} & g_{3}=m^{2} f_{3} & g_{4}=s^{2} f_{4} \\
g_{5}=s f_{5} & g_{6}=s f_{6} & g_{7}=s f_{7} & g_{8}=s f_{8}
\end{array}
$$




$$
\begin{aligned}
& g_{9}=m^{2} s f_{9} \quad g_{10}=m^{2} s f_{10} \quad g_{11}=\lambda_{s} f_{11} \quad g_{12}=\lambda_{s} f_{12} \\
& g_{13}=\lambda_{t} f_{13} \quad g_{14}=m^{2}\left(s f_{14}-4 f_{15}\right) \quad g_{15}=\lambda_{s} f_{15} \quad g_{16}=\lambda_{s} f_{16} \\
& g_{17}=\lambda_{t} f_{17} \quad g_{18}=s^{3} f_{18} \quad g_{19}=s f_{19} \quad g_{20}=s^{2} f_{20} \\
& g_{21}=m^{2} s^{2} f_{21} \quad g_{22}=s \lambda_{s} f_{22} \quad g_{23}=s \lambda_{s} f_{23} \quad g_{24}=\lambda_{s} f_{24} \\
& g_{25}=m^{2} \lambda_{s} f_{25} \quad g_{26}=\lambda_{s} f_{26} \quad g_{27}=\lambda_{s} f_{27} \quad g_{28}=m^{2}\left(s f_{28}-12 f_{27}\right) \\
& g_{29}=\lambda_{s} f_{29} \quad g_{30}=m^{2} s f_{30} \quad g_{31}=s t f_{31} \quad g_{32}=u f_{32} \\
& g_{33}=\lambda_{u} f_{33} \quad g_{34}=s t f_{34} \quad g_{35}=m^{2} s f_{35} \quad g_{36}=s t f_{36} \\
& g_{37}=s\left(f_{37}-f_{17}\right) \quad g_{38}=u f_{38} \quad g_{39}=u f_{39} \quad g_{40}=\lambda_{u} f_{40} \\
& g_{41}=s t f_{41} \quad g_{42}=\lambda_{u} f_{42} \quad g_{43}=s t f_{43} \quad g_{44}=s t f_{44} \\
& g_{45}=s f_{45} \quad g_{46}=s f_{46} \quad g_{47}=s \lambda_{s} f_{47} \quad g_{48}=\lambda_{s} f_{48} \\
& g_{49}=\lambda_{s} f_{49} \quad g_{50}=s \lambda_{s} f_{50} \quad g_{51}=s \lambda_{t} f_{51} \quad g_{52}=\lambda_{s} f_{52} \\
& g_{53}=\lambda_{t} f_{53} \quad g_{54}=m^{2} s f_{54} \quad g_{55}=s \lambda_{s} f_{55} \quad g_{56}=s \lambda_{t} f_{56} \\
& g_{57}=s t f_{57} \quad g_{58}=\lambda_{u} f_{58} \quad g_{59}=m^{2} s f_{59} \quad g_{60}=t f_{60} \\
& g_{61}=s \lambda_{s} f_{61} \quad g_{62}=s \lambda_{t} f_{62} \quad g_{63}=s \lambda_{t} f_{63} \quad g_{64}=s^{2} t f_{64} \\
& g_{65}=s \lambda_{s} f_{65} \quad g_{66}=s u f_{66} \quad g_{67}=s^{2} t f_{67} \quad g_{68}=s u f_{68} \\
& g_{69}=s^{2} t f_{69} \quad g_{70}=s \lambda_{s} f_{70} \quad g_{71}=s^{2} t f_{71} \quad g_{72}=s u f_{72} \\
& g_{73}=s^{2} t f_{73} \quad g_{74}=s \lambda_{u} f_{74} \quad g_{75}=m^{2} s^{2} f_{75} \quad g_{76}=m^{2} s t f_{76} \\
& g_{77}=s^{2} t f_{77} \quad g_{78}=s^{2} f_{78} \quad g_{79}=s^{2} t f_{79} \quad g_{80}=s^{2} \lambda_{t} f_{80} \\
& g_{81}=m^{2}\left(-2 f_{24}-3 f_{26}+4 f_{27}-f_{29}+2 s f_{47}-2 f_{49}-2 \frac{s t}{m^{2}} f_{74}+\frac{s^{2}}{m^{2}} f_{81}-2 s f_{82}\right) \\
& g_{82}=s \lambda_{s} f_{82} \quad g_{83}=s^{3} t f_{83} \quad g_{84}=s^{2} \lambda_{s} f_{84} \quad g_{85}=s^{2} \lambda_{s} f_{85}
\end{aligned}
$$

with $\lambda_{a}$ defined below (3.2). The sparse matrices $M_{i}(i=1, \ldots, 6)$ appearing in the corresponding canonical system (2.31) and (2.32) are given in appendix B.

\section{Boundary conditions}

The generic solutions (2.34) of the canonical systems at two- and three-loop are written in terms of $G$-polylogarithms and constants to be fixed by boundary conditions. The alphabet (2.33) determines the thresholds which appear in the final result. Out of six thresholds only two are physical, since they correspond to the production of massless particles in $s$ - and $t$-channels at $x=0$ and $y=0$. Imposing the regularity of the generic solutions at the unphysical thresholds, namely $x=1, y=1, y=-x, y=1-x$, amounts to ruling out the terms that give rise to divergent behaviours, hence enforcing conditions that unequivocally fix the arbitrary constants.

\subsection{Relations for one-scale integrals}

In general, homogeneous differential equations for single scale integrals carry only information on the scaling behaviour of the solution. Boundary constants for such differential 
equations require the evaluation of the integrals themselves by independent methods. Within a multi-scale problem, such as the one we are considering, integrals may depend on more than one external invariant, and single-scale integrals participate in the regularity conditions of the multi-scale ones. Therefore, these relations can be exploited to determine the arbitrary constants of the single-scale integrals. Alternatively, they can reduce the number of independent single-scale integrals that needs to be independently provided. Therefore, solving multi-scale systems of differential equations yields the simultaneous determination of single- and multi-scale MI's, which are finally expressed in terms of a few single-scale MI's, to be considered as external input.

Let us discuss, as a pedagogical example, the systems of DE's for the two-loop master integrals $g_{2}, g_{5}, g_{8}$ and $g_{11}$, represented by the corresponding topologies in figure 2 , which read,

$$
\begin{aligned}
& \partial_{x} \ddot{Y}=0, \\
& \partial_{x}<=-\frac{2}{x}<, \\
& \partial_{x} \rightleftharpoons=0
\end{aligned}
$$

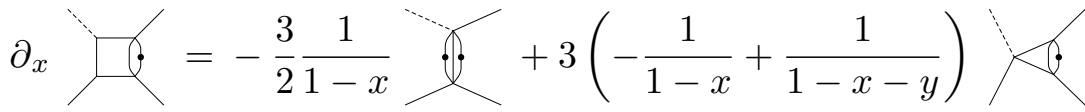

$$
\begin{aligned}
& +3\left(\frac{1}{1-x}-\frac{1}{1-x-y}\right) \nabla-\left(\frac{1}{1-x}+\frac{2}{x}+\frac{1}{1-x-y}\right)>? \text {, }
\end{aligned}
$$

and

$$
\begin{aligned}
\partial_{y} \ddot{Y}= & -\frac{2}{y} \ddot{Y}, \\
\partial_{y}= & 0, \\
\partial_{y}= & -\frac{1}{2 y} \ddot{Y}+\frac{1}{1-y}, \\
& +\left(-\frac{2}{y}-\frac{1}{1-x-y}\right)
\end{aligned}
$$

From the regular behavior of (5.4) and (5.8) at $(1-x) \rightarrow 0,(1-y) \rightarrow 0$ and $(1-x-y) \rightarrow 0$, 
the following relations can be established:

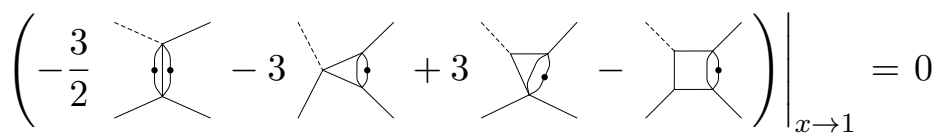

$$
\begin{aligned}
& \sum_{y \rightarrow 1}=0 \\
& \left.(3 \nvdash-3 \&-\gamma)\right|_{y \rightarrow 1-x}=0
\end{aligned}
$$

The zeroth order term of the $\epsilon$-expansion is independent of $x$ and $y$. Therefore, we can combine the equations above in order to find a relation between (the constant terms of) two one-scale integrals,

$$
\left.\right|_{\epsilon^{0}, \text { const. }}=-\left.\frac{1}{4} \ddot{r}\right|_{\epsilon^{0}, \text { const. }}
$$

At higher order in $\epsilon$, these equations acquire a richer structure, because constants coming from the limiting values of the $G$-polylogarithms appear. For the considered example, the relation at the first order in $\epsilon$ is unaltered (the only constant which could appear being imaginary, hence not allowed in the Euclidean region),

$$
\left.\right|_{\epsilon^{1}, \text { const. }}=-\left.\frac{1}{4} \ddot{\gamma}\right|_{\epsilon^{1}, \text { const. }}
$$

but at the second order in $\epsilon$ the relation becomes,

$$
\gamma_{\epsilon^{2}, \text { const. }}=-\left.\frac{1}{4} \dddot{\varkappa}\right|_{\epsilon^{2}, \text { const. }}-\left.\frac{\zeta_{2}}{2} \dddot{\varkappa}\right|_{\epsilon^{2}, \text { const. }} .
$$

Similar relations are systematically established, so that all MI's can be finally determined by providing few simple integrals as external inputs. At two-loop, the only external input is $g_{3}$ in (3.2), which can be independently computed and is given by,

$$
g_{3}=\epsilon^{2} \frac{\Gamma^{2}(1-2 \epsilon) \Gamma^{2}(-\epsilon) \Gamma(1+2 \epsilon)}{\Gamma(1-3 \epsilon) \Gamma^{3}(1-\epsilon) \Gamma^{2}(1+\epsilon)}
$$

while $g_{3}$ and $g_{9}$ in (4.2) are the input integrals for the three-loop MI's, amounting to

$$
\begin{aligned}
& g_{3}=\epsilon^{3} \frac{\Gamma^{3}(1-2 \epsilon) \Gamma^{3}(-\epsilon) \Gamma(1+3 \epsilon)}{\Gamma(1-4 \epsilon) \Gamma^{5}(1-\epsilon) \Gamma^{3}(1+\epsilon)}, \\
& g_{9}=\epsilon^{3} \frac{\Gamma^{2}(1-2 \epsilon) \Gamma^{3}(-\epsilon) \Gamma(1+2 \epsilon)}{\Gamma(1-3 \epsilon) \Gamma^{4}(1-\epsilon) \Gamma^{2}(1+\epsilon)} x^{-\epsilon},
\end{aligned}
$$

where we omit the common normalization factors (2.7). 
We would like to observe that the relations between single-scale integrals, coming from the regularity conditions of multi-scale ones, seem not to belong to the set of IBP identities needed to derive the considered systems of differential equations. In the future, it is worth to investigate whether such relations are truly independent from IBP identities, or if they would arise when considering larger sets of identities for increasing powers of denominators and irreducible scalar products.

The sets of 18 two-loop MI's and of 85 three-loop MI's are collected in two ancillary files 2loopMIs.m and 3loopMIs.m available from http://arxiv.org/abs/1408.3107.

In appendix $\mathrm{C}$, we present only the analytic expression of the three loop $g_{83}$-integral, see figure 5 , that we consider the representative diagram of this work.

\section{Conclusions}

In this article we presented the analytic expressions of the 85 master integrals (MI's) of the three-loop ladder-box topology with one massive leg. Their calculation was performed with the method of differential equations, namely by solving a system of first order differential equations fulfilled by the MI's. The generic solution of the system was obtained in a purely algebraic way, by means of Magnus exponential method, and cast in terms of repeated integrations, according to Dyson series expansion, as recently proposed in ref. [31]. The boundary conditions were provided by the regularity of the solutions at pseudothresholds.

The results of the considered four-leg integrals, as well as of the tower of three- and twoleg master integrals associated to subtopologies (including previously unknown two-scale vertex diagrams), were written as a Taylor expansion in the dimensional regulator parameter $\epsilon=(4-d) / 2$. The coefficients of the series are expressed in terms of uniform weight combinations of multiple polylogarithms and transcendental constants up to weight six.

The considered integrals contribute to the $\mathrm{N}^{3} \mathrm{LO}$ virtual corrections to scattering processes like the three-jet production mediated by vector boson decay, $V^{*} \rightarrow j j j$, as well as the Higgs plus one-jet production in gluon fusion, $p p \rightarrow H j$, and to the three-loop one-particle splitting amplitudes.

\section{Acknowledgments}

We wish to thank Thomas Gehrmann and Lorenzo Tancredi for discussions on multiple polylogarithms and comparisons. We also acknowledge Thomas Gehrmann, Nigel Glover, and Ettore Remiddi for comments on the manuscript. P.M. acknowledges the kind hospitality of the CERN Theory Department during the completion of this work. The work of P.M., U.S. and V.Y. is supported by the Alexander von Humboldt Foundation, in the framework of the Sofja Kovalevskaja Award 2010, endowed by the German Federal Ministry of Education and Research. This research used resources of the National Energy Research Scientific Computing Center, a DOE Office of Science User Facility supported by the Office of Science of the U.S. Department of Energy under Contract No. DE-AC02-05CH11231. 


\section{A Canonical matrices at two-loop}

Here we present the sparse matrices $M_{i}(i=1, \ldots, 6)$ appearing in the canonical system defined in (2.31) and (2.32) obeyed by the MI's (3.2):

$$
M_{1}=\left(\begin{array}{cccccccccccccccccc}
-2 & 0 & 0 & 0 & 0 & 0 & 0 & 0 & 0 & 0 & 0 & 0 & 0 & 0 & 0 & 0 & 0 & 0 \\
0 & 0 & 0 & 0 & 0 & 0 & 0 & 0 & 0 & 0 & 0 & 0 & 0 & 0 & 0 & 0 & 0 & 0 \\
0 & 0 & 0 & 0 & 0 & 0 & 0 & 0 & 0 & 0 & 0 & 0 & 0 & 0 & 0 & 0 & 0 & 0 \\
0 & 0 & 0 & -2 & 0 & 0 & 0 & 0 & 0 & 0 & 0 & 0 & 0 & 0 & 0 & 0 & 0 & 0 \\
0 & 0 & 0 & 0 & -2 & 0 & 0 & 0 & 0 & 0 & 0 & 0 & 0 & 0 & 0 & 0 & 0 & 0 \\
0 & 0 & 0 & 0 & 0 & -1 & 0 & 0 & 0 & 0 & 0 & 0 & 0 & 0 & 0 & 0 & 0 & 0 \\
-\frac{1}{2} & 0 & 0 & 0 & 0 & 0 & 0 & 0 & 0 & 0 & 0 & 0 & 0 & 0 & 0 & 0 & 0 & 0 \\
0 & 0 & 0 & 0 & 0 & 0 & 0 & 0 & 0 & 0 & 0 & 0 & 0 & 0 & 0 & 0 & 0 & 0 \\
0 & 0 & -\frac{1}{2} & 0 & 0 & 0 & 0 & 0 & -1 & 0 & 0 & 0 & 0 & 0 & 0 & 0 & 0 & 0 \\
\frac{1}{2} & 0 & \frac{1}{2} & 0 & 0 & -1 & -2 & 0 & 2 & -2 & 0 & 0 & 0 & 0 & 0 & 0 & 0 & 0 \\
0 & 0 & 0 & 0 & 0 & 0 & 0 & 0 & 0 & 0 & -2 & 0 & 0 & 0 & 0 & 0 & 0 & 0 \\
0 & -\frac{1}{2} & \frac{1}{2} & 0 & 0 & 0 & 0 & 0 & 0 & 0 & 0 & -2 & 0 & 0 & 0 & 0 & 0 & 0 \\
\frac{1}{2} & -\frac{1}{2} & 0 & 0 & 0 & 0 & -2 & 2 & 0 & 0 & 0 & 0 & 2 & 0 & 0 & 0 & 0 & 0 \\
0 & 0 & 0 & 0 & 0 & 0 & 0 & 0 & 0 & 0 & 0 & 0 & 0 & -2 & 0 & 0 & 0 & 0 \\
0 & 0 & 0 & 0 & 0 & 0 & 0 & 0 & 0 & 0 & 0 & 0 & 0 & 0 & -1 & 0 & 0 & 0 \\
-\frac{3}{8} & \frac{3}{8} & -\frac{3}{4} & 0 & 0 & 1 & \frac{5}{2} & -\frac{3}{2} & -2 & 1 & 0 & 0 & \frac{3}{2} & \frac{1}{4} & 0 & -1 & 0 & 0 \\
0 & 0 & 0 & 0 & 0 & 0 & 0 & 0 & 0 & 0 & 0 & 0 & 0 & 0 & 0 & 0 & -2 & 0 \\
-\frac{3}{8} & \frac{3}{8} & -\frac{3}{4} & 0 & 0 & 1 & \frac{5}{2} & -\frac{3}{2} & -2 & 1 & 0 & 0 & \frac{3}{2} & \frac{1}{4} & -1 & 1 & 0 & -2
\end{array}\right)
$$

$$
M_{2}=\left(\begin{array}{cccccccccccccccccc}
0 & 0 & 0 & 0 & 0 & 0 & 0 & 0 & 0 & 0 & 0 & 0 & 0 & 0 & 0 & 0 & 0 & 0 \\
0 & 0 & 0 & 0 & 0 & 0 & 0 & 0 & 0 & 0 & 0 & 0 & 0 & 0 & 0 & 0 & 0 & 0 \\
0 & 0 & 0 & 0 & 0 & 0 & 0 & 0 & 0 & 0 & 0 & 0 & 0 & 0 & 0 & 0 & 0 & 0 \\
0 & 0 & 0 & 0 & 0 & 0 & 0 & 0 & 0 & 0 & 0 & 0 & 0 & 0 & 0 & 0 & 0 & 0 \\
0 & 0 & 0 & 0 & 0 & 0 & 0 & 0 & 0 & 0 & 0 & 0 & 0 & 0 & 0 & 0 & 0 & 0 \\
0 & 0 & 0 & 0 & 0 & 0 & 0 & 0 & 0 & 0 & 0 & 0 & 0 & 0 & 0 & 0 & 0 & 0 \\
0 & 0 & 0 & 0 & 0 & 0 & -1 & 0 & 0 & 0 & 0 & 0 & 0 & 0 & 0 & 0 & 0 & 0 \\
0 & 0 & 0 & 0 & 0 & 0 & 0 & 0 & 0 & 0 & 0 & 0 & 0 & 0 & 0 & 0 & 0 & 0 \\
0 & 0 & 0 & 0 & 0 & 0 & 0 & 0 & -1 & 0 & 0 & 0 & 0 & 0 & 0 & 0 & 0 & 0 \\
0 & 0 & 0 & 0 & 0 & 0 & 0 & 0 & 0 & 2 & 0 & 0 & 0 & 0 & 0 & 0 & 0 & 0 \\
0 & \frac{3}{2} & 0 & 0 & 0 & 0 & 0 & 0 & 0 & 0 & 2 & 0 & 0 & 0 & 0 & 0 & 0 & 0 \\
\frac{1}{2} & 0 & -\frac{1}{2} & 0 & 0 & 0 & 0 & 0 & 0 & 0 & 0 & 2 & 0 & 0 & 0 & 0 & 0 & 0 \\
-\frac{1}{8} & -\frac{1}{8} & 0 & 0 & 0 & 0 & -\frac{1}{2} & -\frac{1}{2} & 0 & 0 & 0 & 0 & -\frac{1}{2} & -\frac{1}{4} & 0 & 0 & 0 & 0 \\
\frac{3}{4} & \frac{3}{4} & 0 & 0 & 0 & 0 & -3 & 3 & 0 & 0 & 0 & 0 & 3 & \frac{3}{2} & 0 & 0 & 0 & 0 \\
0 & \frac{3}{2} & 0 & 0 & 0 & 0 & 0 & 0 & 0 & 0 & 0 & 0 & 0 & 0 & 1 & 0 & 0 & 0 \\
\frac{3}{8} & -\frac{3}{8} & \frac{3}{4} & 0 & 0 & -1 & -\frac{5}{2} & \frac{3}{2} & 2 & -1 & 0 & 0 & -\frac{3}{2} & -\frac{1}{4} & 0 & 1 & 0 & 0 \\
\frac{3}{2} & \frac{3}{2} & -\frac{3}{2} & 0 & 0 & 0 & 0 & 0 & 0 & 0 & 2 & 6 & 0 & -1 & 2 & 0 & 2 & 0 \\
-\frac{9}{8} & -\frac{15}{8} & \frac{3}{2} & -1 & 3 & 1 & -\frac{3}{2} & -\frac{3}{2} & 0 & -3 & -2 & -9 & \frac{3}{2} & \frac{5}{4} & -2 & 0 & -1 & -1
\end{array}\right)
$$




$$
M_{3}=\left(\begin{array}{cccccccccccccccccc}
0 & 0 & 0 & 0 & 0 & 0 & 0 & 0 & 0 & 0 & 0 & 0 & 0 & 0 & 0 & 0 & 0 & 0 \\
0 & -2 & 0 & 0 & 0 & 0 & 0 & 0 & 0 & 0 & 0 & 0 & 0 & 0 & 0 & 0 & 0 & 0 \\
0 & 0 & 0 & 0 & 0 & 0 & 0 & 0 & 0 & 0 & 0 & 0 & 0 & 0 & 0 & 0 & 0 & 0 \\
0 & 0 & 0 & 0 & 0 & 0 & 0 & 0 & 0 & 0 & 0 & 0 & 0 & 0 & 0 & 0 & 0 & 0 \\
0 & 0 & 0 & 0 & 0 & 0 & 0 & 0 & 0 & 0 & 0 & 0 & 0 & 0 & 0 & 0 & 0 & 0 \\
0 & 0 & 0 & 0 & 0 & 0 & 0 & 0 & 0 & 0 & 0 & 0 & 0 & 0 & 0 & 0 & 0 & 0 \\
0 & 0 & 0 & 0 & 0 & 0 & 0 & 0 & 0 & 0 & 0 & 0 & 0 & 0 & 0 & 0 & 0 & 0 \\
0 & -\frac{1}{2} & 0 & 0 & 0 & 0 & 0 & 0 & 0 & 0 & 0 & 0 & 0 & 0 & 0 & 0 & 0 & 0 \\
0 & 0 & 0 & 0 & 0 & 0 & 0 & 0 & 0 & 0 & 0 & 0 & 0 & 0 & 0 & 0 & 0 & 0 \\
0 & 0 & 0 & 0 & 0 & 0 & 0 & 0 & 0 & 0 & 0 & 0 & 0 & 0 & 0 & 0 & 0 & 0 \\
0 & 0 & 0 & 0 & 0 & 0 & 0 & 0 & 0 & 0 & -2 & 0 & 0 & 0 & 0 & 0 & 0 & 0 \\
-\frac{1}{2} & 0 & \frac{1}{2} & 0 & 0 & 0 & 0 & 0 & 0 & 0 & 0 & -2 & 0 & 0 & 0 & 0 & 0 & 0 \\
-\frac{1}{2} & \frac{1}{2} & 0 & 0 & 0 & 0 & 2 & -2 & 0 & 0 & 0 & 0 & -2 & 0 & 0 & 0 & 0 & 0 \\
0 & 0 & 0 & 0 & 0 & 0 & 0 & 0 & 0 & 0 & 0 & 0 & 0 & -2 & 0 & 0 & 0 & 0 \\
0 & 0 & 0 & 0 & 0 & 0 & 0 & 0 & 0 & 0 & 0 & 0 & 0 & 0 & -2 & 0 & 0 & 0 \\
\frac{3}{8} & -\frac{3}{8} & \frac{3}{4} & 0 & 0 & -1 & -\frac{1}{2} & \frac{3}{2} & 1 & 1 & 0 & 0 & -\frac{3}{2} & \frac{3}{4} & -1 & 1 & 0 & 0 \\
-\frac{3}{2} & -\frac{3}{2} & \frac{3}{2} & 0 & 0 & 0 & 0 & 0 & 0 & 0 & -2 & -6 & 0 & 0 & 0 & 0 & -2 & 0 \\
\frac{9}{8} & \frac{15}{8} & -\frac{3}{2} & 1 & -3 & -1 & -\frac{1}{2} & \frac{3}{2} & 1 & 1 & 2 & 9 & -\frac{3}{2} & -\frac{5}{4} & 2 & 0 & 1 & 1
\end{array}\right)
$$

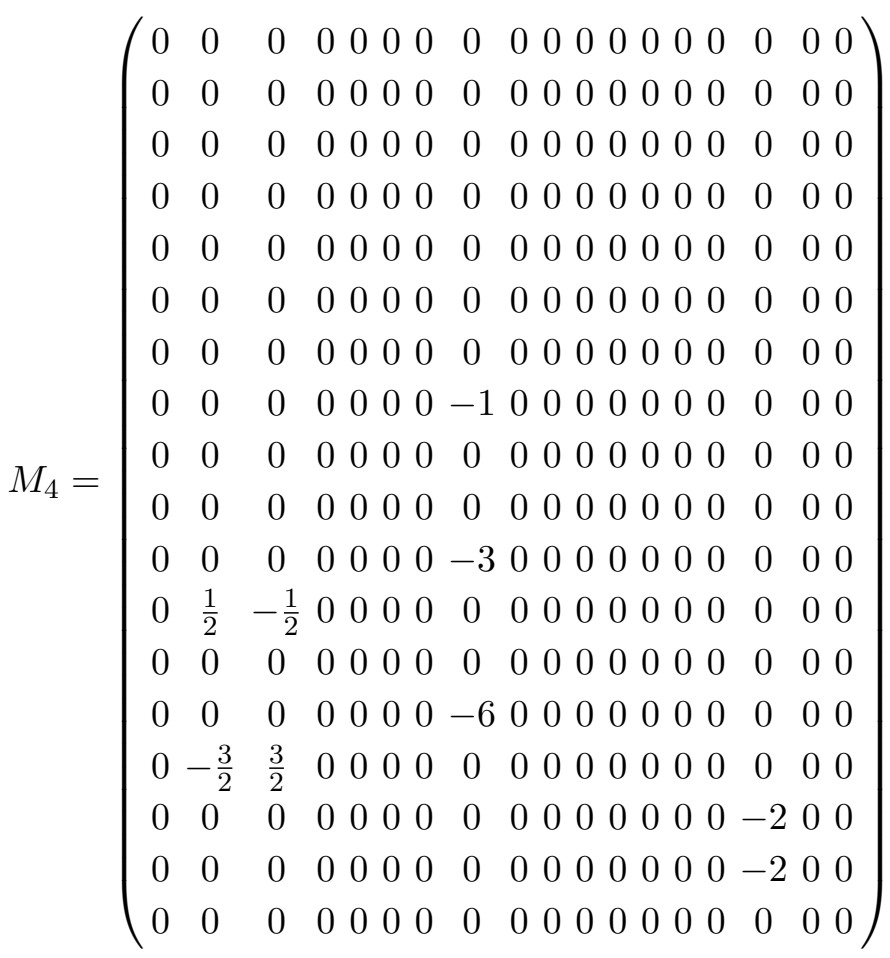


$\left(M_{5}\right)_{13,13}=4$ is the only non-vanishing entry of $M_{5}$.

$$
M_{6}=\left(\begin{array}{cccccccccccccccccc}
0 & 0 & 0 & 0 & 0 & 0 & 0 & 0 & 0 & 0 & 0 & 0 & 0 & 0 & 0 & 0 & 0 & 0 \\
0 & 0 & 0 & 0 & 0 & 0 & 0 & 0 & 0 & 0 & 0 & 0 & 0 & 0 & 0 & 0 & 0 & 0 \\
0 & 0 & 0 & 0 & 0 & 0 & 0 & 0 & 0 & 0 & 0 & 0 & 0 & 0 & 0 & 0 & 0 & 0 \\
0 & 0 & 0 & 0 & 0 & 0 & 0 & 0 & 0 & 0 & 0 & 0 & 0 & 0 & 0 & 0 & 0 & 0 \\
0 & 0 & 0 & 0 & 0 & 0 & 0 & 0 & 0 & 0 & 0 & 0 & 0 & 0 & 0 & 0 & 0 & 0 \\
0 & 0 & 0 & 0 & 0 & 0 & 0 & 0 & 0 & 0 & 0 & 0 & 0 & 0 & 0 & 0 & 0 & 0 \\
0 & 0 & 0 & 0 & 0 & 0 & 0 & 0 & 0 & 0 & 0 & 0 & 0 & 0 & 0 & 0 & 0 & 0 \\
0 & 0 & 0 & 0 & 0 & 0 & 0 & 0 & 0 & 0 & 0 & 0 & 0 & 0 & 0 & 0 & 0 & 0 \\
0 & 0 & 0 & 0 & 0 & 0 & 0 & 0 & 0 & 0 & 0 & 0 & 0 & 0 & 0 & 0 & 0 & 0 \\
0 & 0 & 0 & 0 & 0 & 0 & 0 & 0 & 0 & 0 & 0 & 0 & 0 & 0 & 0 & 0 & 0 & 0 \\
0 & 0 & 0 & 0 & -3 & 0 & 0 & 3 & 0 & 0 & 1 & 0 & 0 & 0 & 0 & 0 & 0 & 0 \\
0 & 0 & 0 & 0 & 0 & 0 & 0 & 0 & 0 & 0 & 0 & 2 & 0 & 0 & 0 & 0 & 0 & 0 \\
-\frac{1}{8} & -\frac{1}{8} & 0 & 0 & 0 & 0 & -\frac{1}{2} & -\frac{1}{2} & 0 & 0 & 0 & 0 & -\frac{1}{2} & -\frac{1}{4} & 0 & 0 & 0 & 0 \\
\frac{3}{4} & \frac{3}{4} & 0 & 0 & 0 & 0 & 3 & 3 & 0 & 0 & 0 & 0 & 3 & \frac{3}{2} & 0 & 0 & 0 & 0 \\
0 & \frac{3}{2} & 0 & 0 & 0 & 0 & 0 & 0 & 3 & 0 & 0 & 0 & 0 & 0 & 1 & 0 & 0 & 0 \\
\frac{3}{8} & -\frac{3}{8} & \frac{3}{4} & 0 & 0 & -1 & -\frac{5}{2} & \frac{3}{2} & 2 & -1 & 0 & 0 & -\frac{3}{2} & -\frac{1}{4} & 0 & 1 & 0 & 0 \\
3 & \frac{3}{2} & -3 & -2 & 0 & 2 & 2 & 0 & -4 & 2 & 2 & 6 & 0 & -1 & 2 & 0 & 2 & 2 \\
-\frac{9}{8} & -\frac{15}{8} & \frac{3}{2} & 1 & 3 & -1 & \frac{1}{2} & -\frac{3}{2} & -1 & -1 & -2 & -9 & \frac{3}{2} & \frac{5}{4} & -2 & 0 & -1 & -1
\end{array}\right)
$$

\section{B Canonical matrices at three-loop}

Here we present the sparse matrices $M_{i}(i=1, \ldots, 6)$ appearing in the canonical system defined in (2.31) and (2.32) obeyed by the MI's (4.2). We write each matrix in block form,

$$
M_{i}=\left(\begin{array}{cc}
M_{i}^{a} & 0_{43 \times 42} \\
M_{i}^{b} & M_{i}^{c}
\end{array}\right),
$$


where $0_{43 \times 42}$ is the null $43 \times 42$ matrix.

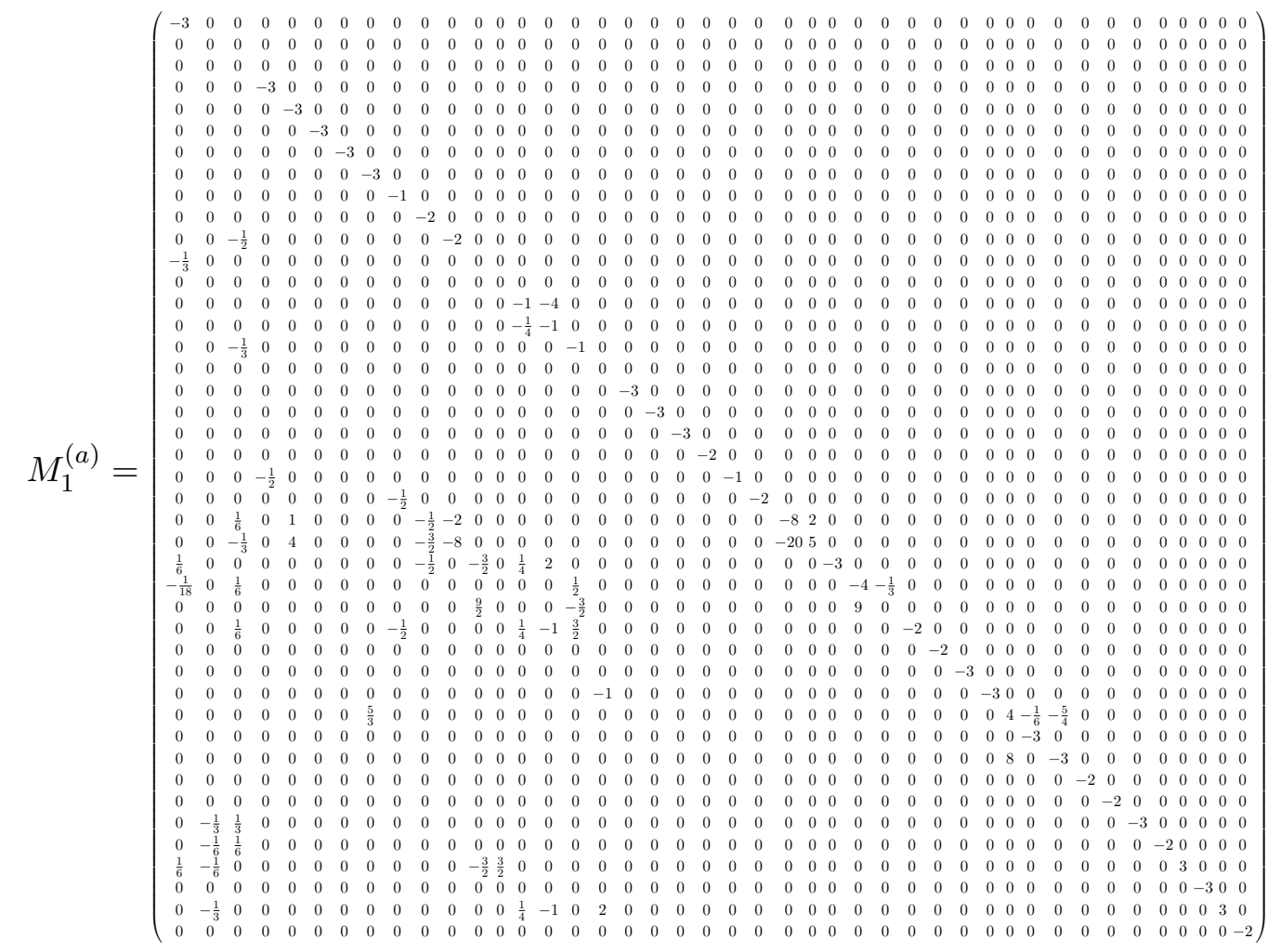

(B.2)

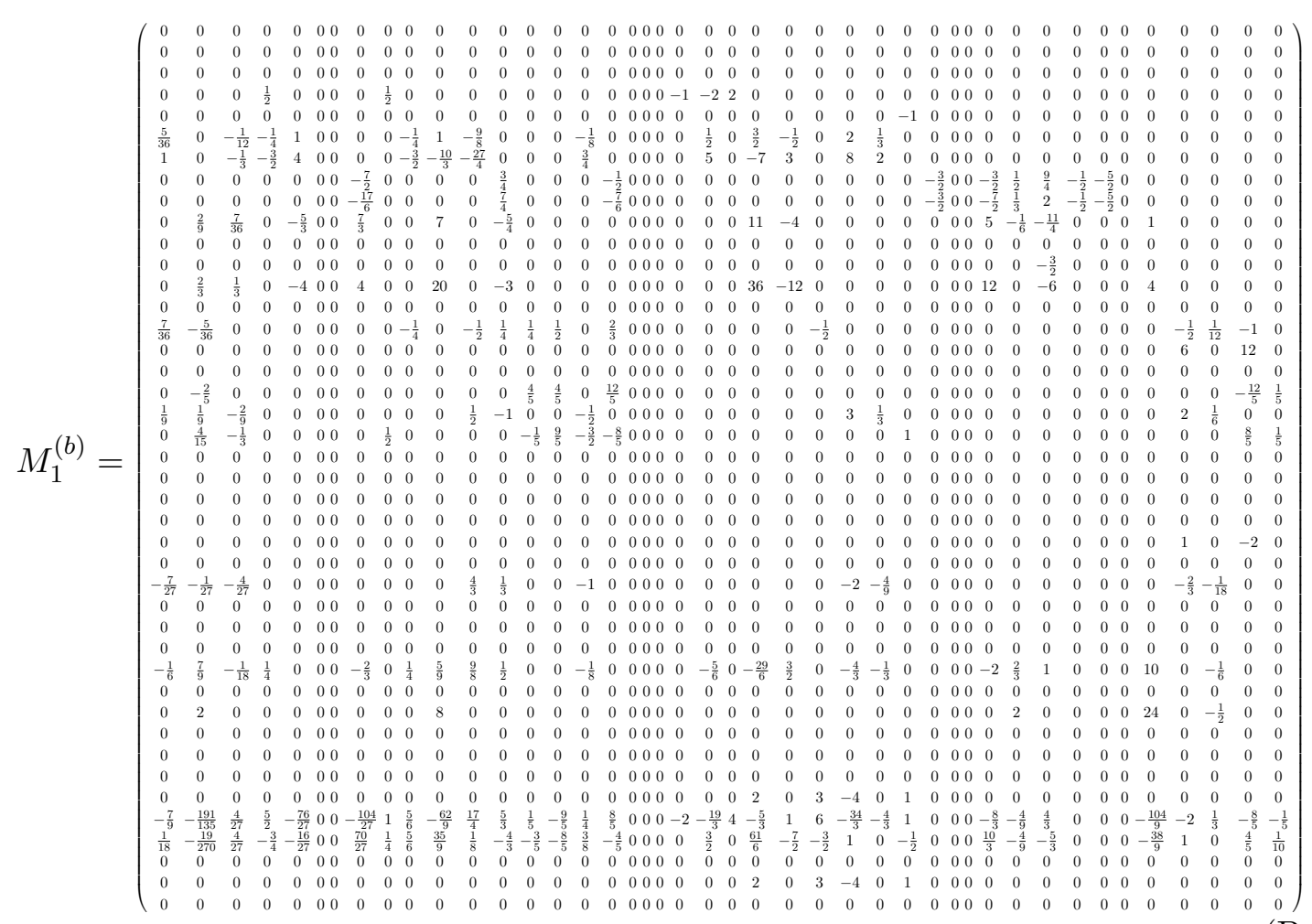




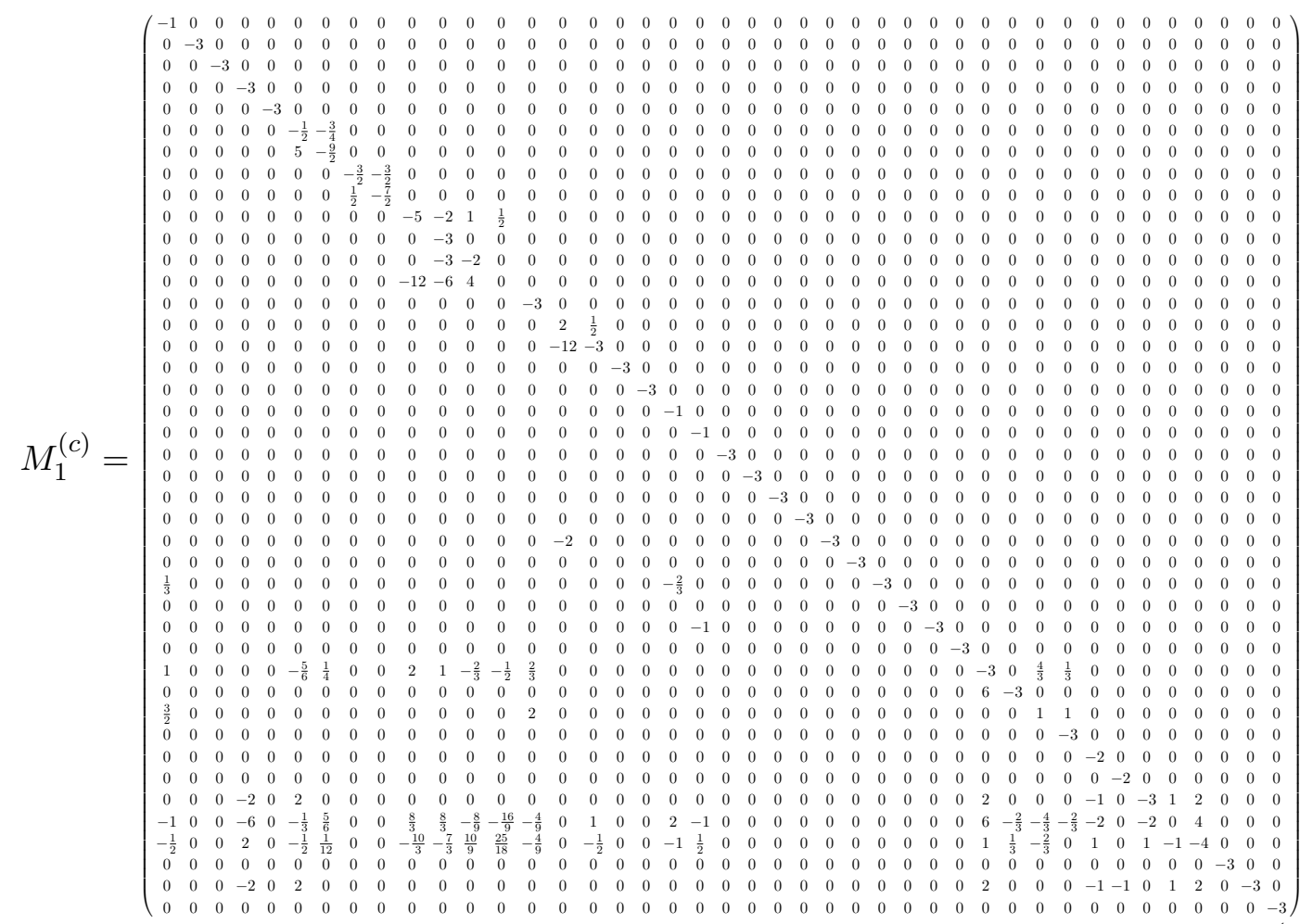

(B.4)

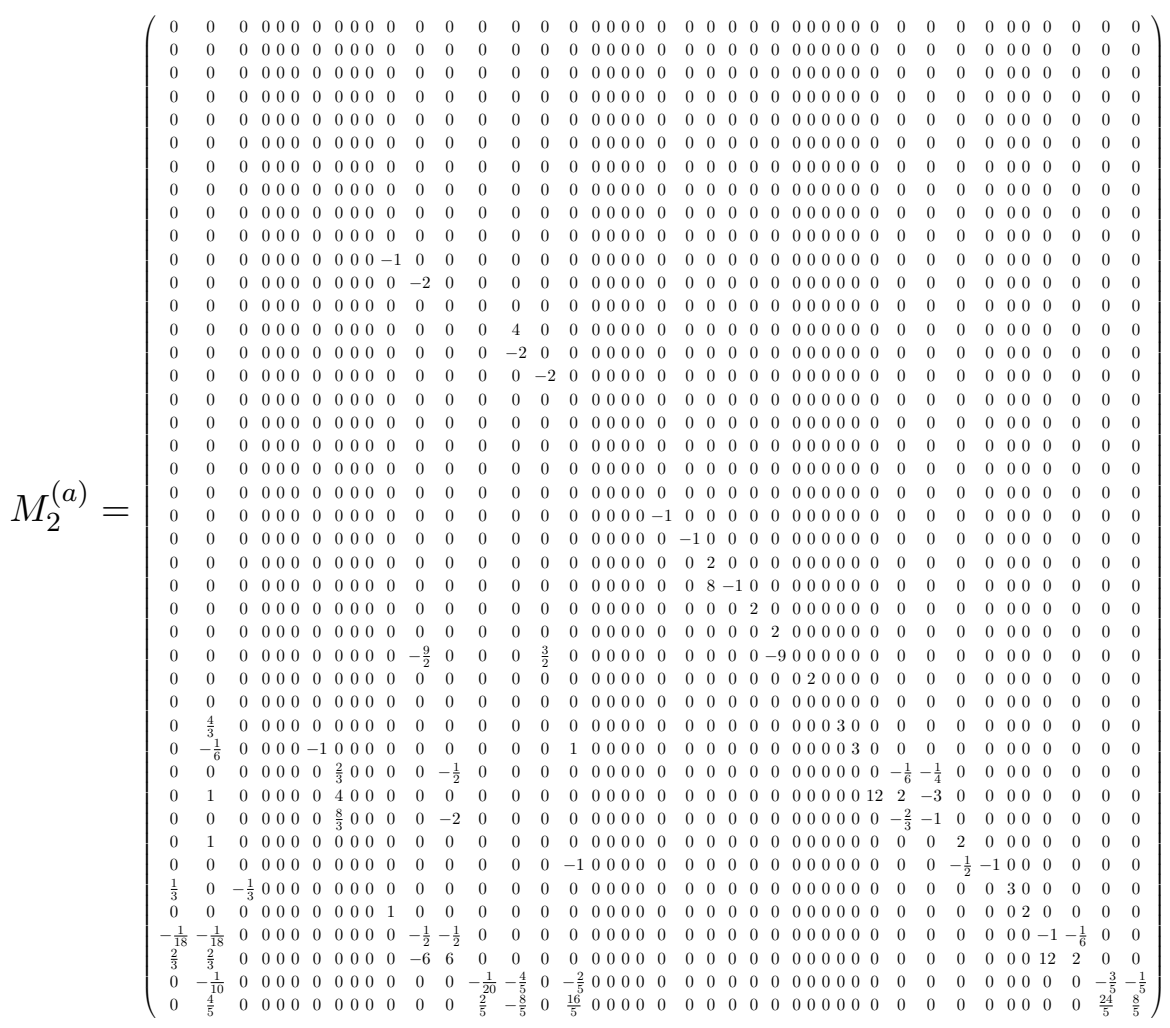

(B.5) 


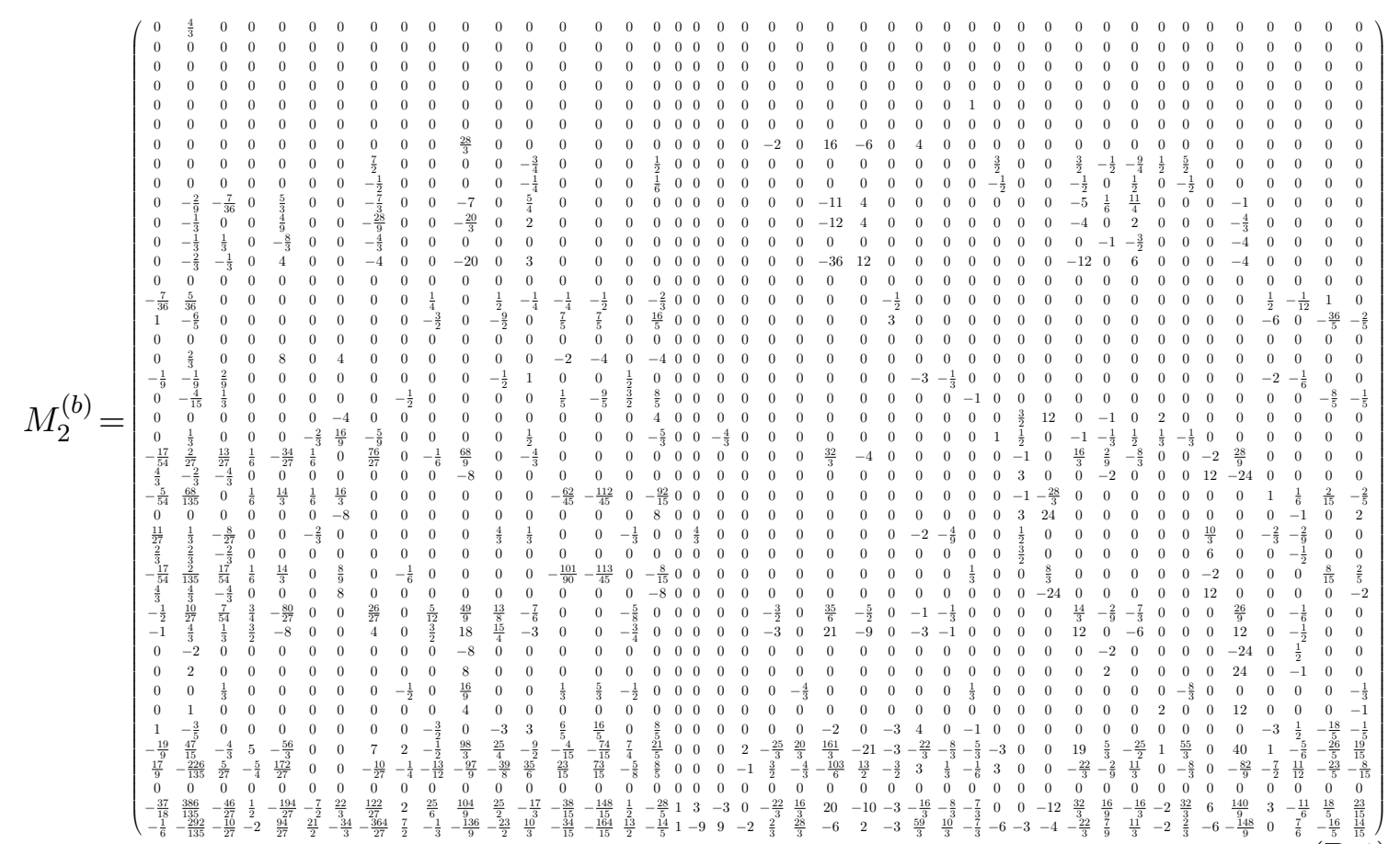

(B.6)

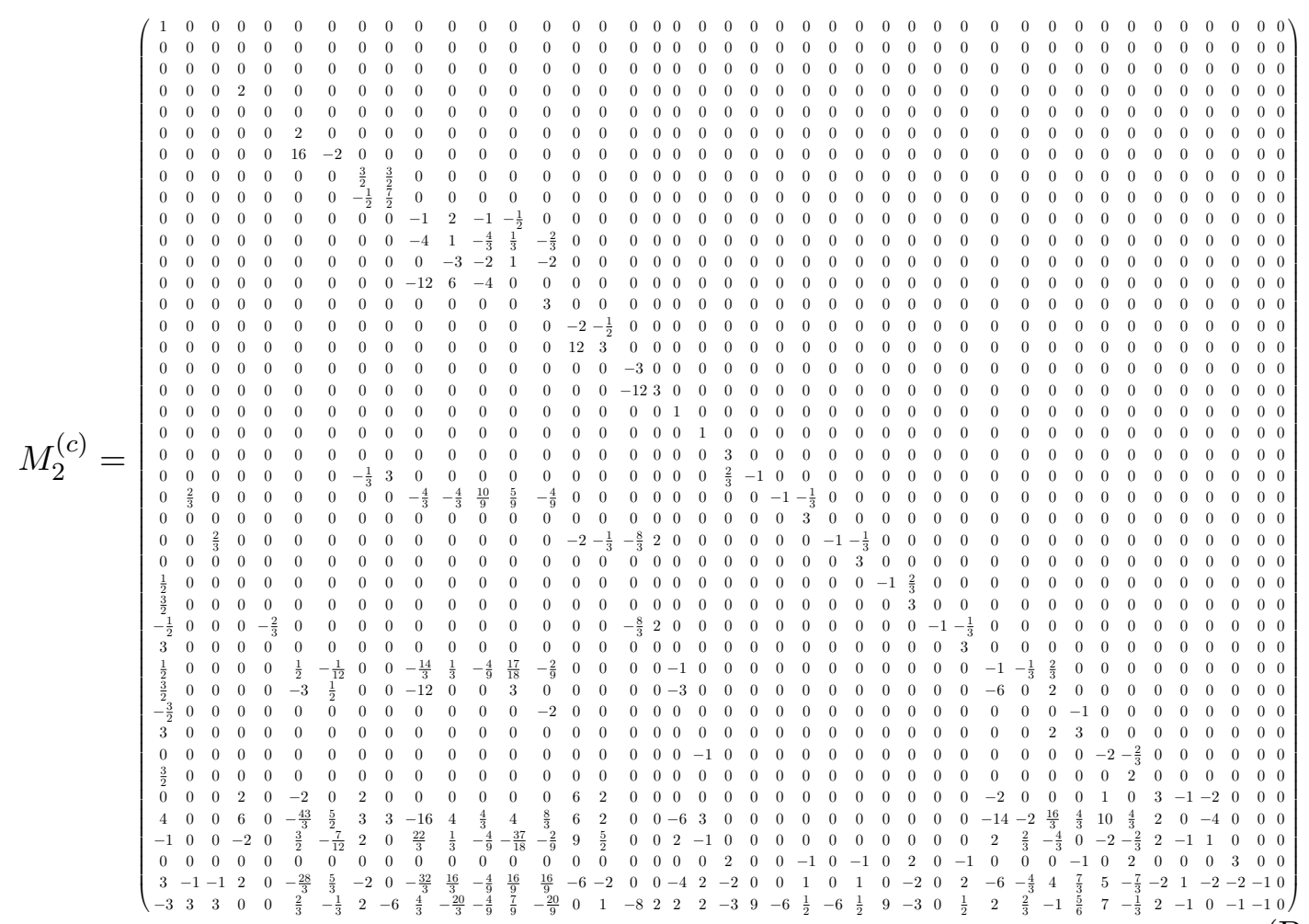

(B.7) 


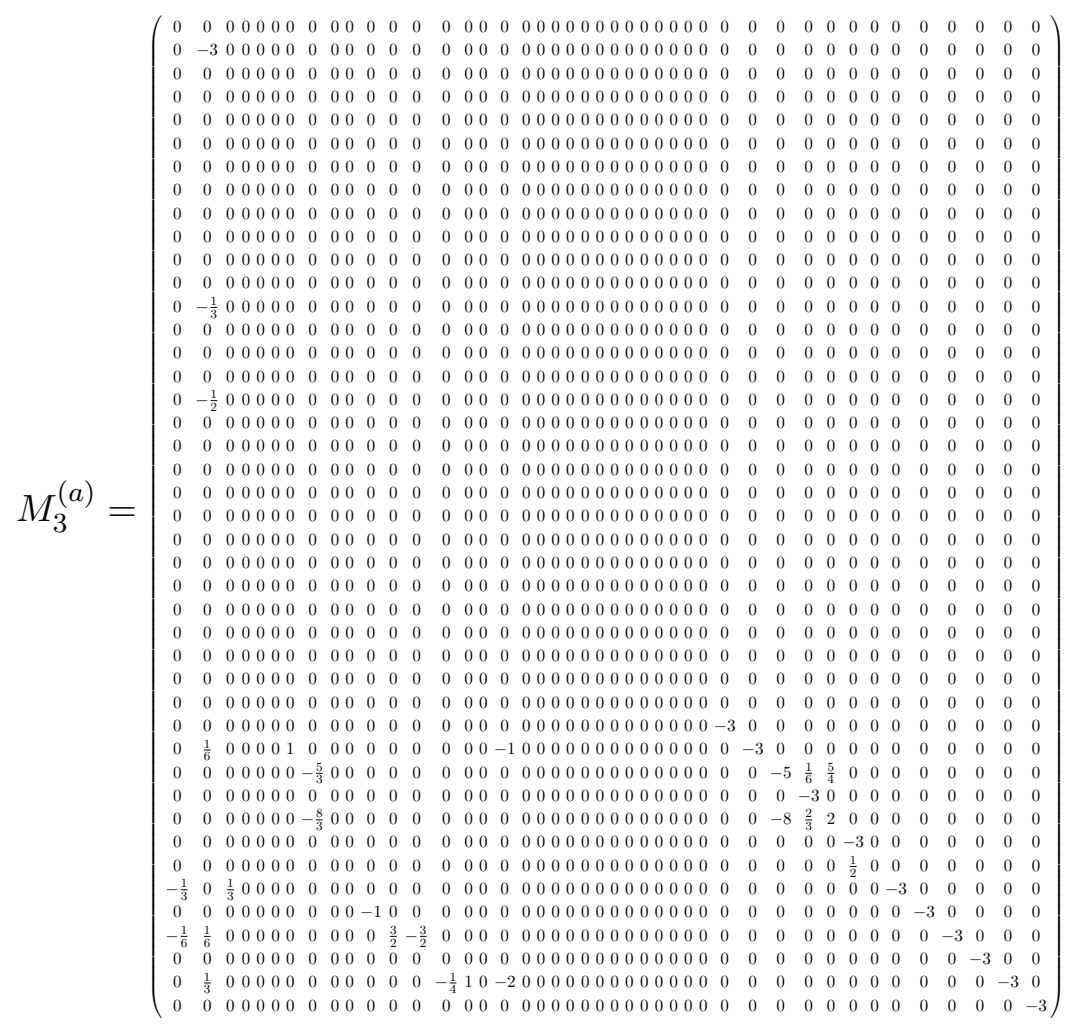

(B.8)

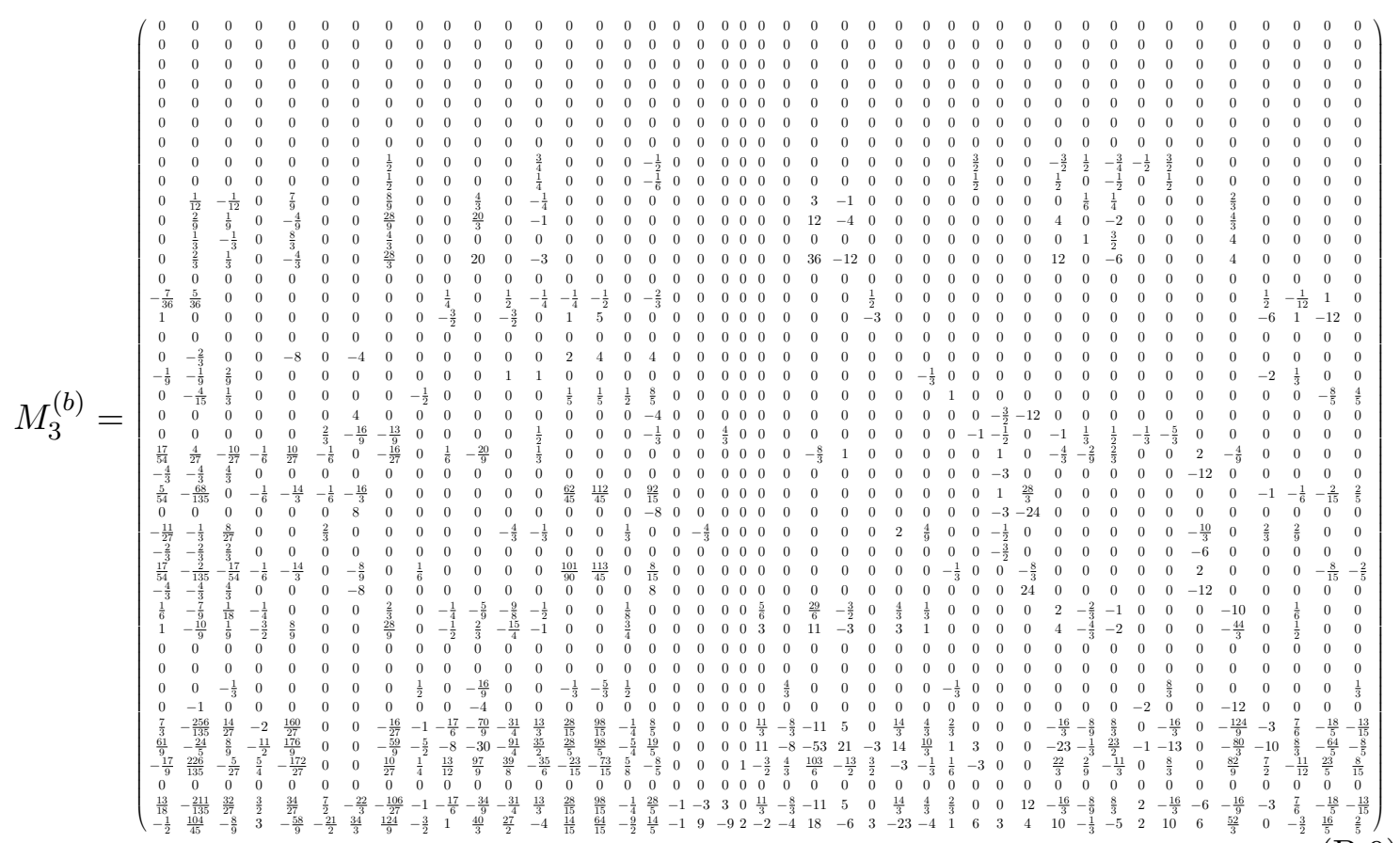




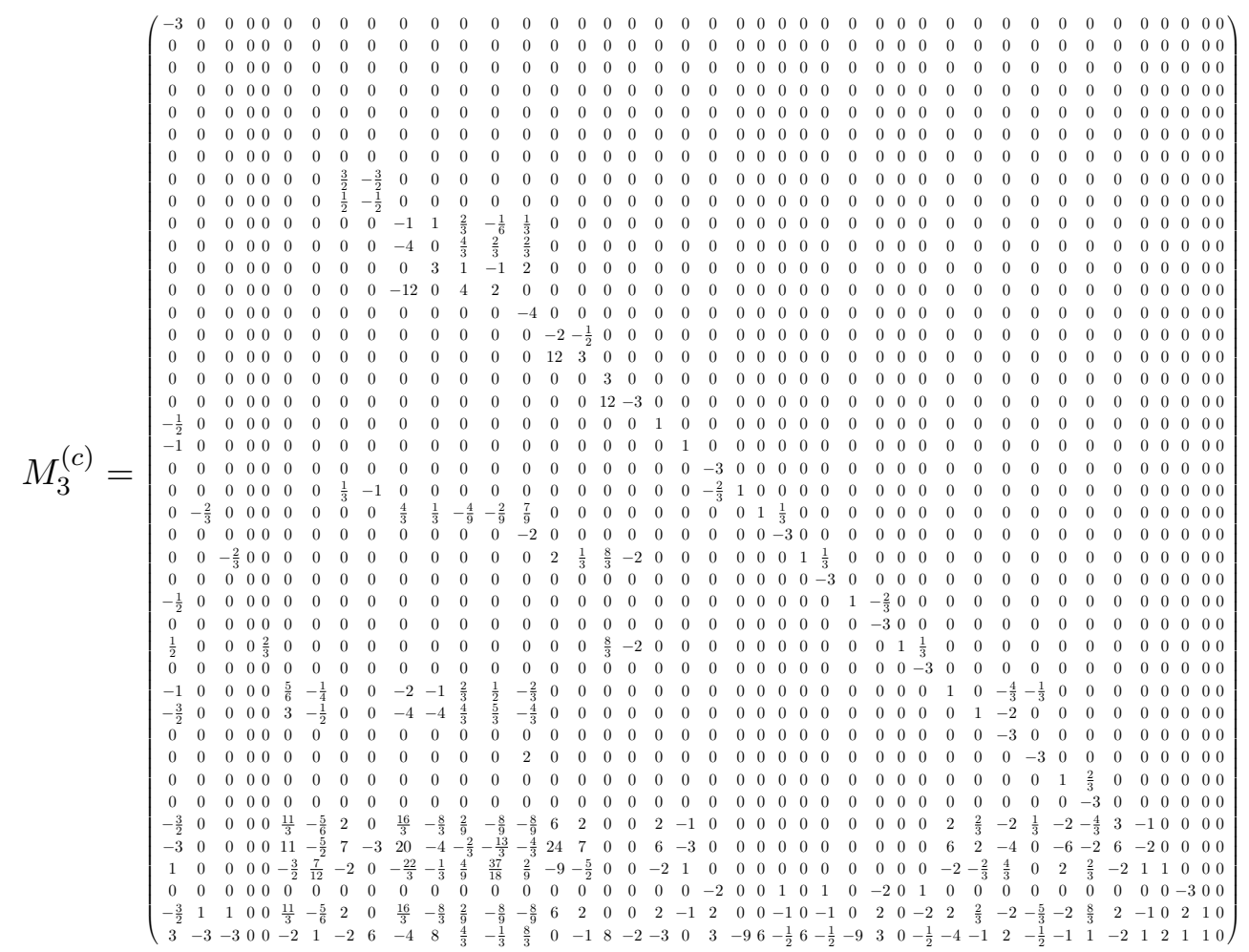

(B.10)

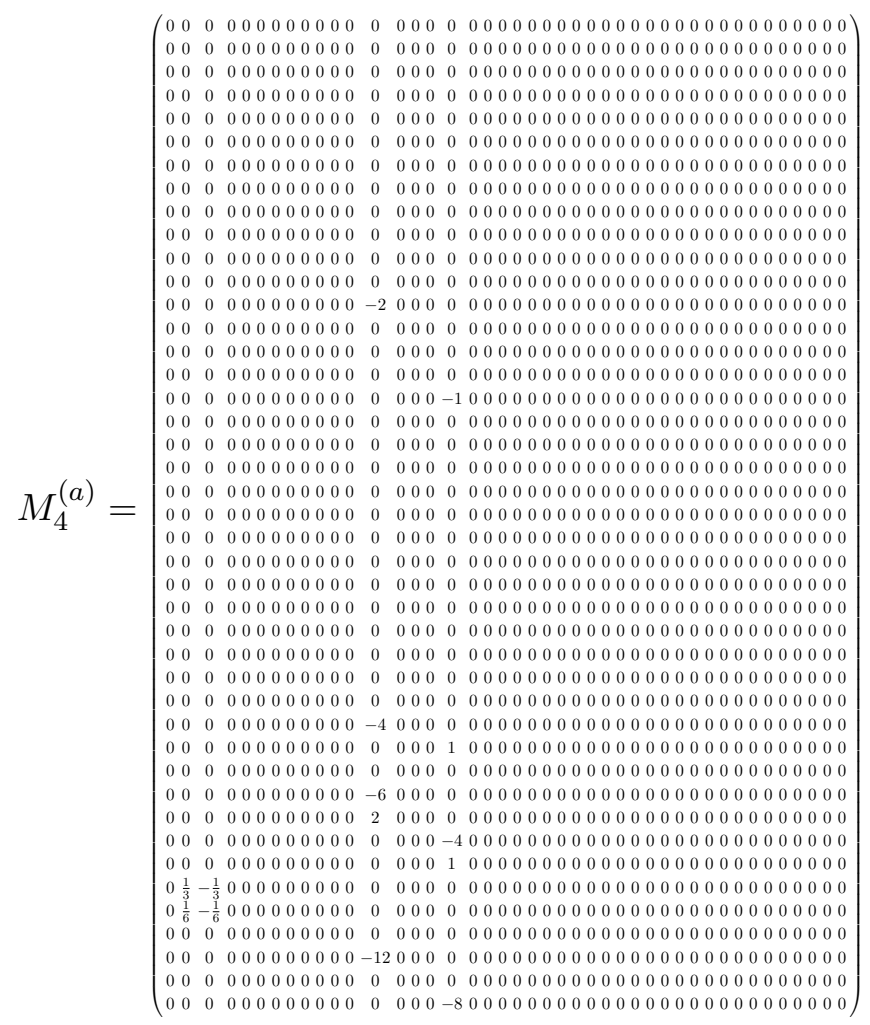




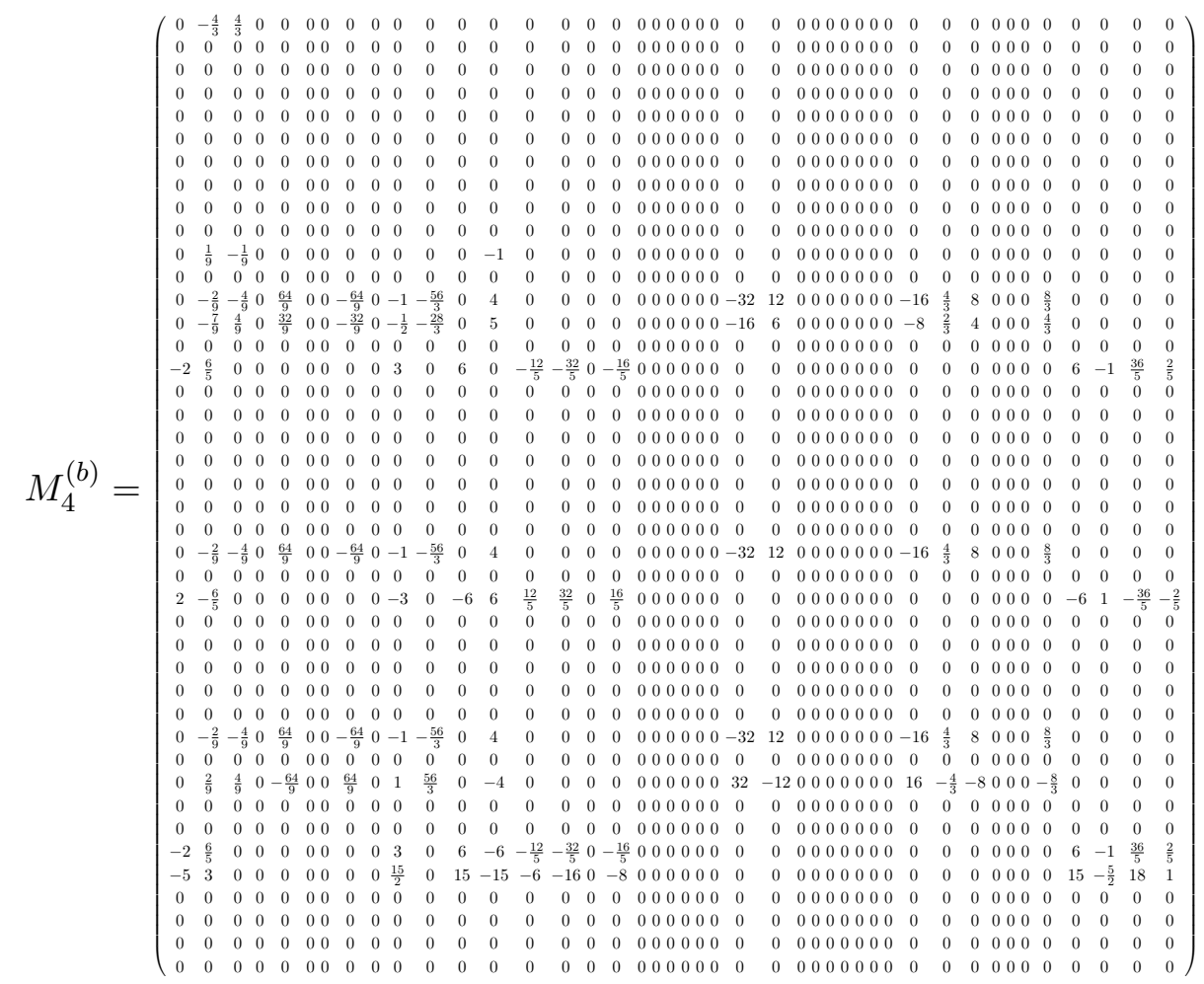

(B.12)

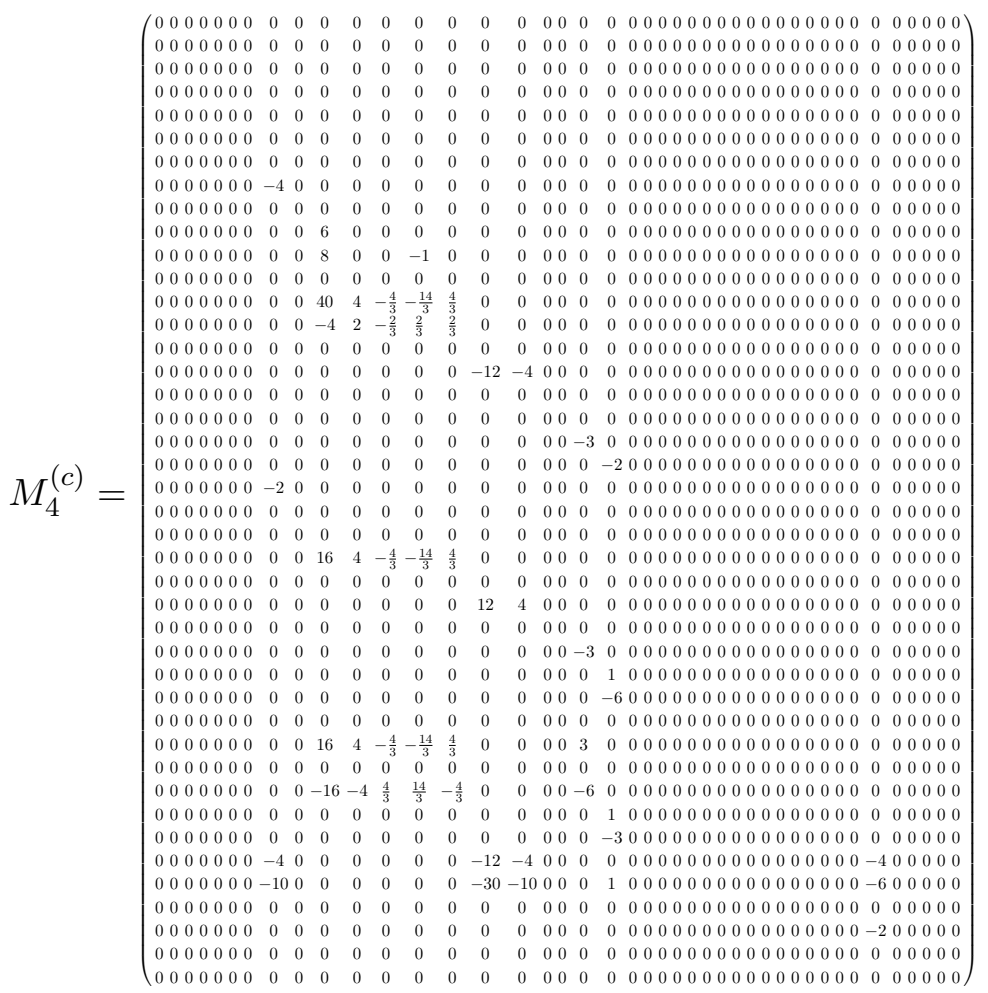

The matrix $M_{5}$ is mainly composed of zeroes and we only list its non-vanishing entries: $\left(M_{5}\right)_{59,58}=-12,\left(M_{5}\right)_{68,42}=\left(M_{5}\right)_{68,58}=-2,\left(M_{5}\right)_{58,42}=-1,\left(M_{5}\right)_{58,40}=\left(M_{5}\right)_{60,42}=$ $\left(M_{5}\right)_{68,40}=1,\left(M_{5}\right)_{74,74}=\left(M_{5}\right)_{81,74}=2,\left(M_{5}\right)_{33,33}=\left(M_{5}\right)_{58,58}=4,\left(M_{5}\right)_{42,42}=5$, 


$$
\left(M_{5}\right)_{40,40}=\left(M_{5}\right)_{59,40}=\left(M_{5}\right)_{75,74}=\left(M_{5}\right)_{76,74}=6,\left(M_{5}\right)_{35,33}=8,\left(M_{5}\right)_{59,42}=12 .
$$

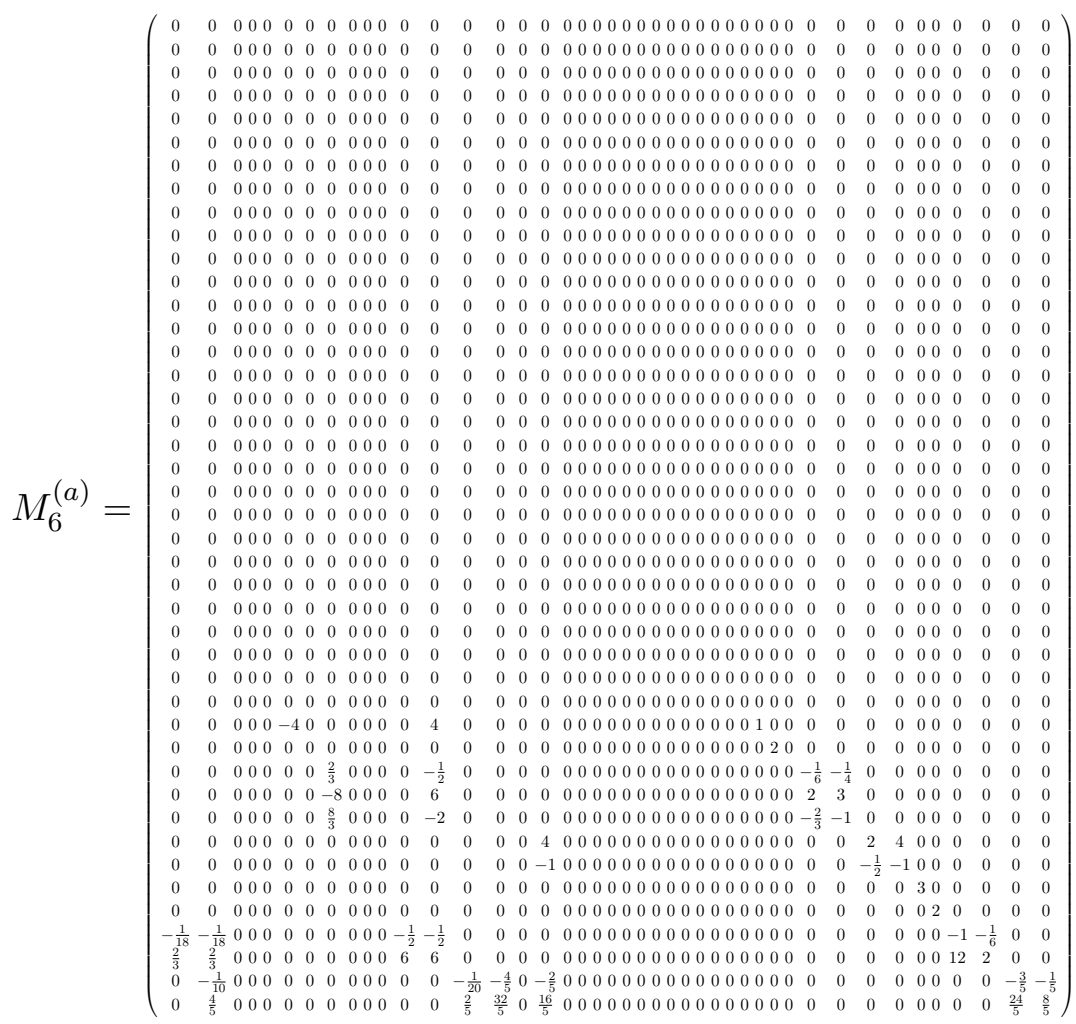

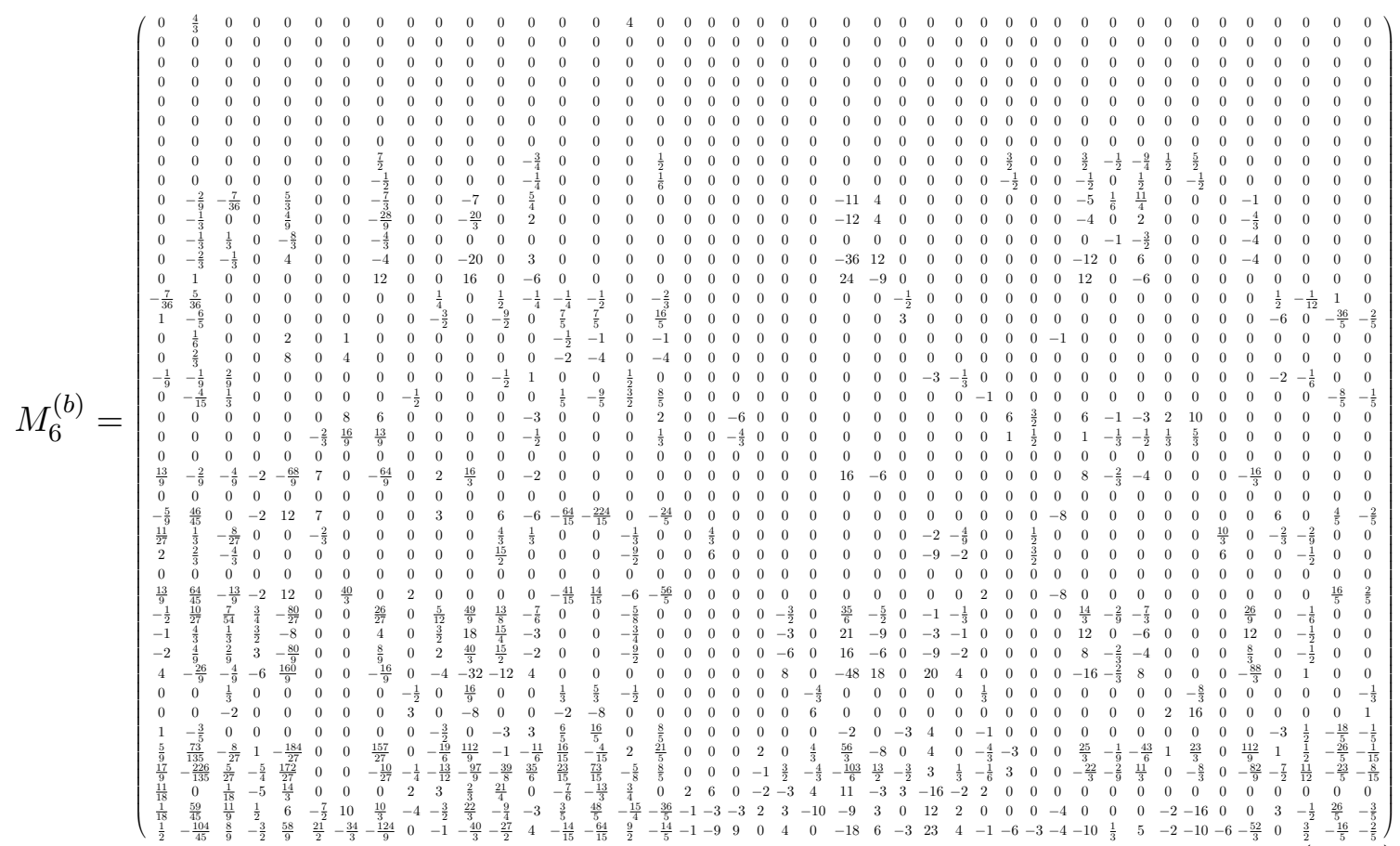




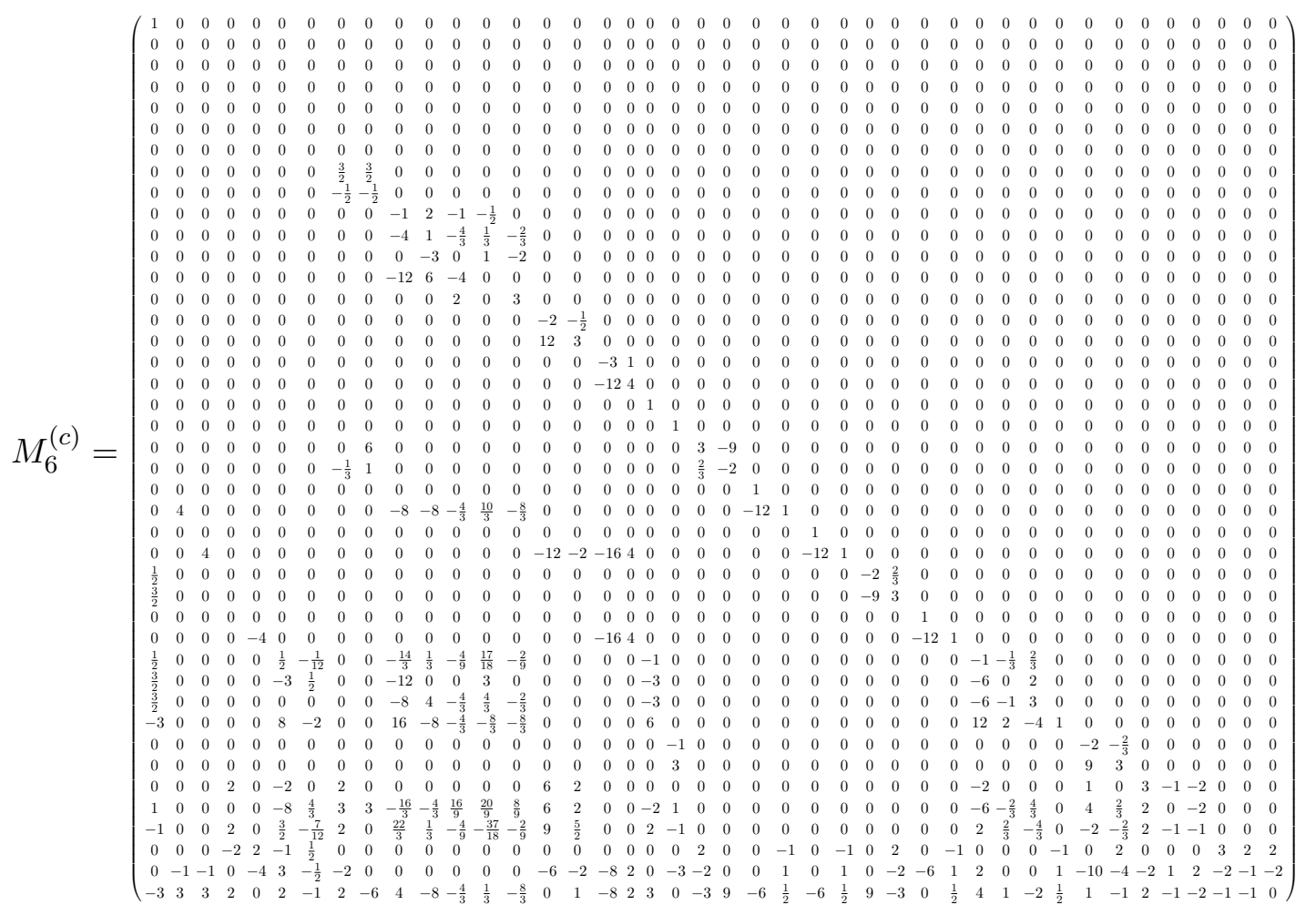

(B.16)

\section{Three-loop scalar ladder}

As an example, we provide the explicit result for the scalar ladder in (4.2),

$$
g_{83}=\sum_{n=0}^{6} \epsilon^{n} g_{83}^{(n)}+\mathcal{O}\left(\epsilon^{7}\right) .
$$

In order to reduce the clutter, we use here the notation

$$
G_{w_{1}, \ldots, w_{n}}(x) \equiv G\left(w_{1}, \ldots, w_{n} ; x\right) .
$$

The coefficients of the $\epsilon$-expansion of $g_{83}$ are:

$$
\begin{aligned}
g_{83}^{(0)}= & \frac{2}{9} \\
g_{83}^{(1)}= & -\frac{2}{3}\left(G_{0}(x)+G_{0}(y)\right) \\
g_{83}^{(2)}= & 2 G_{0}(x) G_{0}(y)+2 G_{0,0}(x)-\frac{7}{6} G_{1,0}(x)+2 G_{0,0}(y)-\frac{1}{6} G_{1,0}(y)+\frac{3 \zeta_{2}}{2} \\
g_{83}^{(3)}= & -6 G_{0,0}(x) G_{0}(y)-6 G_{0}(x) G_{0,0}(y)+\frac{7}{2} G_{1,0}(x) G_{0}(y)-\frac{1}{2} G_{1,0}(x) G_{1-x}(y) \\
& +\frac{1}{2} G_{0}(x) G_{1-x, 0}(y)-\frac{1}{2} G_{1-x, 1,0}(y)-6 G_{0,0,0}(x)+\frac{7}{2} G_{0,1,0}(x)+\frac{35}{6} G_{1,0,0}(x) \\
& -\frac{17}{6} G_{1,1,0}(x)-6 G_{0,0,0}(y)+\frac{11}{6} G_{0,1,0}(y)+\frac{1}{2} G_{1,0,0}(y)+\frac{2}{3} G_{1,1,0}(y) \\
& +\frac{1}{6} \zeta_{2}\left(3 G_{1-x}(y)-27 G_{0}(x)+17 G_{1}(x)-27 G_{0}(y)-4 G_{1}(y)\right)-\frac{5 \zeta_{3}}{9}
\end{aligned}
$$




$$
\begin{aligned}
& g_{83}^{(4)}=18 G_{0,0}(x) G_{0,0}(y)-\frac{21}{2} G_{1,0}(x) G_{0,0}(y)+\frac{11}{2} G_{1,0}(x) G_{0,1-x}(y)+G_{0,0}(x) G_{1,0}(y) \\
& -G_{1,0}(x) G_{1,0}(y)+G_{1,0}(x) G_{1,1-x}(y)-\frac{5}{2} G_{0,0}(x) G_{1-x, 0}(y)+\frac{1}{2} G_{1,0}(x) G_{1-x, 0}(y) \\
& -\frac{1}{2} G_{1,0}(x) G_{1-x, 1-x}(y)+18 G_{0,0,0}(x) G_{0}(y)+18 G_{0}(x) G_{0,0,0}(y)-\frac{21}{2} G_{0,1,0}(x) G_{0}(y) \\
& -G_{0,1,0}(x) G_{1}(y)+\frac{5}{2} G_{0,1,0}(x) G_{1-x}(y)-\frac{11}{2} G_{0}(x) G_{0,1-x, 0}(y)-\frac{35}{2} G_{1,0,0}(x) G_{0}(y) \\
& +\frac{5}{2} G_{1,0,0}(x) G_{1-x}(y)+\frac{17}{2} G_{1,1,0}(x) G_{0}(y)+G_{1,1,0}(x) G_{1}(y)+\frac{1}{2} G_{1,1,0}(x) G_{1-x}(y) \\
& -G_{0}(x) G_{1,1-x, 0}(y)-\frac{3}{2} G_{0}(x) G_{1-x, 0,0}(y)+\frac{1}{2} G_{0}(x) G_{1-x, 1-x, 0}(y)+\frac{11}{2} G_{0,1-x, 1,0}(y) \\
& +G_{1,1-x, 1,0}(y)+\frac{3}{2} G_{1-x, 0,1,0}(y)+\frac{3}{2} G_{1-x, 1,0,0}(y)+2 G_{1-x, 1,1,0}(y)-\frac{1}{2} G_{1-x, 1-x, 1,0}(y) \\
& +18 G_{0,0,0,0}(x)-\frac{21}{2} G_{0,0,1,0}(x)-\frac{35}{2} G_{0,1,0,0}(x)+\frac{17}{2} G_{0,1,1,0}(x)-\frac{133}{6} G_{1,0,0,0}(x) \\
& +\frac{25}{2} G_{1,0,1,0}(x)+\frac{109}{6} G_{1,1,0,0}(x)-\frac{43}{6} G_{1,1,1,0}(x)+18 G_{0,0,0,0}(y)-\frac{71}{6} G_{0,0,1,0}(y) \\
& -\frac{11}{2} G_{0,1,0,0}(y)-\frac{10}{3} G_{0,1,1,0}(y)-\frac{3}{2} G_{1,0,0,0}(y)+\frac{2}{3} G_{1,0,1,0}(y)-2 G_{1,1,0,0}(y) \\
& -\frac{8}{3} G_{1,1,1,0}(y)+\frac{1}{6} \zeta_{2}\left(81 G_{0}(x) G_{0}(y)-51 G_{1}(x) G_{0}(y)+6 G_{0}(x) G_{1}(y)-6 G_{1}(x) G_{1}(y)\right. \\
& -3 G_{0}(x) G_{1-x}(y)-3 G_{1}(x) G_{1-x}(y)-33 G_{0,1-x}(y)-6 G_{1,1-x}(y)-3 G_{1-x, 0}(y) \\
& -12 G_{1-x, 1}(y)+3 G_{1-x, 1-x}(y)+81 G_{0,0}(x)-51 G_{0,1}(x)-81 G_{1,0}(x)+43 G_{1,1}(x) \\
& \left.+81 G_{0,0}(y)+20 G_{0,1}(y)+16 G_{1,1}(y)\right)+\frac{1}{3} \zeta_{3}\left(-9 G_{1-x}(y)+5 G_{0}(x)+G_{1}(x)\right. \\
& \left.+5 G_{0}(y)-5 G_{1}(y)\right)+\frac{163 \zeta_{4}}{8} \\
& g_{83}^{(5)}=-54 G_{0,0}(y) G_{0,0,0}(x)-5 G_{1,0}(y) G_{0,0,0}(x)+\frac{19}{2} G_{1-x, 0}(y) G_{0,0,0}(x) \\
& -54 G_{0,0}(x) G_{0,0,0}(y)+\frac{63}{2} G_{1,0}(x) G_{0,0,0}(y)-\frac{71}{2} G_{1,0}(x) G_{0,0,1-x}(y) \\
& +\frac{63}{2} G_{0,0}(y) G_{0,1,0}(x)+5 G_{0,1}(y) G_{0,1,0}(x)-\frac{43}{2} G_{0,1-x}(y) G_{0,1,0}(x) \\
& +3 G_{1,0}(y) G_{0,1,0}(x)+4 G_{1,1}(y) G_{0,1,0}(x)-5 G_{1,1-x}(y) G_{0,1,0}(x) \\
& -\frac{3}{2} G_{1-x, 0}(y) G_{0,1,0}(x)-3 G_{1-x, 1}(y) G_{0,1,0}(x)+\frac{5}{2} G_{1-x, 1-x}(y) G_{0,1,0}(x) \\
& -5 G_{0,0}(x) G_{0,1,0}(y)+5 G_{1,0}(x) G_{0,1,0}(y)-5 G_{1,0}(x) G_{0,1,1-x}(y) \\
& +\frac{43}{2} G_{0,0}(x) G_{0,1-x, 0}(y)-\frac{21}{2} G_{1,0}(x) G_{0,1-x, 0}(y)+\frac{23}{2} G_{1,0}(x) G_{0,1-x, 1-x}(y) \\
& +\frac{105}{2} G_{0,0}(y) G_{1,0,0}(x)-\frac{43}{2} G_{0,1-x}(y) G_{1,0,0}(x)+5 G_{1,0}(y) G_{1,0,0}(x) \\
& -5 G_{1,1-x}(y) G_{1,0,0}(x)-\frac{17}{2} G_{1-x, 0}(y) G_{1,0,0}(x)+\frac{5}{2} G_{1-x, 1-x}(y) G_{1,0,0}(x) \\
& -3 G_{0,0}(x) G_{1,0,0}(y)+3 G_{1,0}(x) G_{1,0,0}(y)-G_{1,0}(x) G_{1,0,1-x}(y) \\
& -\frac{51}{2} G_{0,0}(y) G_{1,1,0}(x)-5 G_{0,1}(y) G_{1,1,0}(x)+\frac{9}{2} G_{0,1-x}(y) G_{1,1,0}(x) \\
& -3 G_{1,0}(y) G_{1,1,0}(x)-4 G_{1,1}(y) G_{1,1,0}(x)+3 G_{1,1-x}(y) G_{1,1,0}(x) \\
& -\frac{1}{2} G_{1-x, 0}(y) G_{1,1,0}(x)+3 G_{1-x, 1}(y) G_{1,1,0}(x)+\frac{1}{2} G_{1-x, 1-x}(y) G_{1,1,0}(x)
\end{aligned}
$$




$$
\begin{aligned}
& -4 G_{0,0}(x) G_{1,1,0}(y)+4 G_{1,0}(x) G_{1,1,0}(y)-4 G_{1,0}(x) G_{1,1,1-x}(y) \\
& +5 G_{0,0}(x) G_{1,1-x, 0}(y)-3 G_{1,0}(x) G_{1,1-x, 0}(y)+G_{1,0}(x) G_{1,1-x, 1-x}(y) \\
& +\frac{15}{2} G_{0,0}(x) G_{1-x, 0,0}(y)-\frac{3}{2} G_{1,0}(x) G_{1-x, 0,0}(y)+\frac{15}{2} G_{1,0}(x) G_{1-x, 0,1-x}(y) \\
& +3 G_{0,0}(x) G_{1-x, 1,0}(y)-3 G_{1,0}(x) G_{1-x, 1,0}(y)+3 G_{1,0}(x) G_{1-x, 1,1-x}(y) \\
& -\frac{5}{2} G_{0,0}(x) G_{1-x, 1-x, 0}(y)+\frac{1}{2} G_{1,0}(x) G_{1-x, 1-x, 0}(y)-\frac{1}{2} G_{1,0}(x) G_{1-x, 1-x, 1-x}(y) \\
& -54 G_{0}(y) G_{0,0,0,0}(x)-54 G_{0}(x) G_{0,0,0,0}(y)+\frac{63}{2} G_{0}(y) G_{0,0,1,0}(x) \\
& +5 G_{1}(y) G_{0,0,1,0}(x)-\frac{19}{2} G_{1-x}(y) G_{0,0,1,0}(x)+\frac{71}{2} G_{0}(x) G_{0,0,1-x, 0}(y) \\
& +\frac{105}{2} G_{0}(y) G_{0,1,0,0}(x)+5 G_{1}(y) G_{0,1,0,0}(x)-\frac{25}{2} G_{1-x}(y) G_{0,1,0,0}(x) \\
& \text { - } \frac{51}{2} G_{0}(y) G_{0,1,1,0}(x)-3 G_{1}(y) G_{0,1,1,0}(x)-\frac{3}{2} G_{1-x}(y) G_{0,1,1,0}(x) \\
& +5 G_{0}(x) G_{0,1,1-x, 0}(y)+\frac{33}{2} G_{0}(x) G_{0,1-x, 0,0}(y)-\frac{23}{2} G_{0}(x) G_{0,1-x, 1-x, 0}(y) \\
& +\frac{133}{2} G_{0}(y) G_{1,0,0,0}(x)-\frac{19}{2} G_{1-x}(y) G_{1,0,0,0}(x)-\frac{75}{2} G_{0}(y) G_{1,0,1,0}(x) \\
& -5 G_{1}(y) G_{1,0,1,0}(x)+\frac{5}{2} G_{1-x}(y) G_{1,0,1,0}(x)+G_{0}(x) G_{1,0,1-x, 0}(y) \\
& \text { - } \frac{109}{2} G_{0}(y) G_{1,1,0,0}(x)-5 G_{1}(y) G_{1,1,0,0}(x)+\frac{19}{2} G_{1-x}(y) G_{1,1,0,0}(x) \\
& +\frac{43}{2} G_{0}(y) G_{1,1,1,0}(x)+3 G_{1}(y) G_{1,1,1,0}(x)+\frac{3}{2} G_{1-x}(y) G_{1,1,1,0}(x) \\
& +4 G_{0}(x) G_{1,1,1-x, 0}(y)+3 G_{0}(x) G_{1,1-x, 0,0}(y)-G_{0}(x) G_{1,1-x, 1-x, 0}(y) \\
& +\frac{9}{2} G_{0}(x) G_{1-x, 0,0,0}(y)-\frac{15}{2} G_{0}(x) G_{1-x, 0,1-x, 0}(y)-3 G_{0}(x) G_{1-x, 1,1-x, 0}(y) \\
& -\frac{3}{2} G_{0}(x) G_{1-x, 1-x, 0,0}(y)+\frac{1}{2} G_{0}(x) G_{1-x, 1-x, 1-x, 0}(y)-54 G_{0,0,0,0,0}(x) \\
& -54 G_{0,0,0,0,0}(y)+\frac{63}{2} G_{0,0,0,1,0}(x)+\frac{359}{6} G_{0,0,0,1,0}(y)+\frac{105}{2} G_{0,0,1,0,0}(x) \\
& +\frac{71}{2} G_{0,0,1,0,0}(y)-\frac{51}{2} G_{0,0,1,1,0}(x)+\frac{28}{3} G_{0,0,1,1,0}(y)-\frac{71}{2} G_{0,0,1-x, 1,0}(y) \\
& +\frac{133}{2} G_{0,1,0,0,0}(x)+\frac{33}{2} G_{0,1,0,0,0}(y)-\frac{75}{2} G_{0,1,0,1,0}(x)-\frac{14}{3} G_{0,1,0,1,0}(y) \\
& -\frac{109}{2} G_{0,1,1,0,0}(x)+10 G_{0,1,1,0,0}(y)+\frac{43}{2} G_{0,1,1,1,0}(x)+\frac{22}{3} G_{0,1,1,1,0}(y) \\
& -5 G_{0,1,1-x, 1,0}(y)-\frac{35}{2} G_{0,1-x, 0,1,0}(y)-\frac{33}{2} G_{0,1-x, 1,0,0}(y)-10 G_{0,1-x, 1,1,0}(y) \\
& +\frac{23}{2} G_{0,1-x, 1-x, 1,0}(y)+\frac{455}{6} G_{1,0,0,0,0}(x)+\frac{9}{2} G_{1,0,0,0,0}(y)-\frac{91}{2} G_{1,0,0,1,0}(x) \\
& -\frac{8}{3} G_{1,0,0,1,0}(y)-\frac{133}{2} G_{1,0,1,0,0}(x)-2 G_{1,0,1,0,0}(y)+\frac{185}{6} G_{1,0,1,1,0}(x) \\
& -\frac{2}{3} G_{1,0,1,1,0}(y)-G_{1,0,1-x, 1,0}(y)-\frac{467}{6} G_{1,1,0,0,0}(x)+6 G_{1,1,0,0,0}(y) \\
& +\frac{265}{6} G_{1,1,0,1,0}(x)-\frac{8}{3} G_{1,1,0,1,0}(y)+\frac{335}{6} G_{1,1,1,0,0}(x)+8 G_{1,1,1,0,0}(y) \\
& -\frac{113}{6} G_{1,1,1,1,0}(x)+\frac{32}{3} G_{1,1,1,1,0}(y)-4 G_{1,1,1-x, 1,0}(y)-G_{1,1-x, 0,1,0}(y) \\
& -3 G_{1,1-x, 1,0,0}(y)-4 G_{1,1-x, 1,1,0}(y)+G_{1,1-x, 1-x, 1,0}(y)-\frac{19}{2} G_{1-x, 0,0,1,0}(y)
\end{aligned}
$$




$$
\begin{aligned}
& -\frac{9}{2} G_{1-x, 0,1,0,0}(y)+\frac{15}{2} G_{1-x, 0,1-x, 1,0}(y)-\frac{9}{2} G_{1-x, 1,0,0,0}(y)+2 G_{1-x, 1,0,1,0}(y) \\
& -6 G_{1-x, 1,1,0,0}(y)-8 G_{1-x, 1,1,1,0}(y)+3 G_{1-x, 1,1-x, 1,0}(y)+\frac{3}{2} G_{1-x, 1-x, 0,1,0}(y) \\
& +\frac{3}{2} G_{1-x, 1-x, 1,0,0}(y)+2 G_{1-x, 1-x, 1,1,0}(y)-\frac{1}{2} G_{1-x, 1-x, 1-x, 1,0}(y) \\
& +\frac{1}{6} \zeta_{2}\left(-243 G_{0,0}(x) G_{0}(y)-6 G_{0,0}(x) G_{1}(y)-3 G_{0,0}(x) G_{1-x}(y)-243 G_{0}(x) G_{0,0}(y)\right. \\
& +153 G_{1}(x) G_{0,0}(y)+153 G_{0,1}(x) G_{0}(y)+18 G_{0,1}(x) G_{1}(y)+9 G_{0,1}(x) G_{1-x}(y) \\
& -30 G_{0}(x) G_{0,1}(y)+30 G_{1}(x) G_{0,1}(y)+69 G_{0}(x) G_{0,1-x}(y)-27 G_{1}(x) G_{0,1-x}(y) \\
& +243 G_{1,0}(x) G_{0}(y)+6 G_{1,0}(x) G_{1}(y)-21 G_{1,0}(x) G_{1-x}(y)-18 G_{0}(x) G_{1,0}(y) \\
& +18 G_{1}(x) G_{1,0}(y)-129 G_{1,1}(x) G_{0}(y)-18 G_{1,1}(x) G_{1}(y)-9 G_{1,1}(x) G_{1-x}(y) \\
& -24 G_{0}(x) G_{1,1}(y)+24 G_{1}(x) G_{1,1}(y)+6 G_{0}(x) G_{1,1-x}(y)-18 G_{1}(x) G_{1,1-x}(y) \\
& +27 G_{0}(x) G_{1-x, 0}(y)+3 G_{1}(x) G_{1-x, 0}(y)+18 G_{0}(x) G_{1-x, 1}(y)-18 G_{1}(x) G_{1-x, 1}(y) \\
& -3 G_{0}(x) G_{1-x, 1-x}(y)-3 G_{1}(x) G_{1-x, 1-x}(y)+213 G_{0,0,1-x}(y)+30 G_{0,1,1-x}(y) \\
& +63 G_{0,1-x, 0}(y)+60 G_{0,1-x, 1}(y)-69 G_{0,1-x, 1-x}(y)+6 G_{1,0,1-x}(y)+24 G_{1,1,1-x}(y) \\
& +18 G_{1,1-x, 0}(y)+24 G_{1,1-x, 1}(y)-6 G_{1,1-x, 1-x}(y)+9 G_{1-x, 0,0}(y)-45 G_{1-x, 0,1-x}(y) \\
& +48 G_{1-x, 1,1}(y)-18 G_{1-x, 1,1-x}(y)-3 G_{1-x, 1-x, 0}(y)-12 G_{1-x, 1-x, 1}(y) \\
& +3 G_{1-x, 1-x, 1-x}(y)-243 G_{0,0,0}(x)+153 G_{0,0,1}(x)+243 G_{0,1,0}(x)-129 G_{0,1,1}(x) \\
& +303 G_{1,0,0}(x)-185 G_{1,0,1}(x)-259 G_{1,1,0}(x)+113 G_{1,1,1}(x)-243 G_{0,0,0}(y) \\
& \left.-56 G_{0,0,1}(y)-44 G_{0,1,1}(y)+4 G_{1,0,1}(y)-64 G_{1,1,1}(y)\right)+\frac{1}{3} \zeta_{3}\left(-15 G_{0}(x) G_{0}(y)\right. \\
& -3 G_{1}(x) G_{0}(y)-6 G_{0}(x) G_{1}(y)+6 G_{1}(x) G_{1}(y)+48 G_{0}(x) G_{1-x}(y)+9 G_{1}(x) G_{1-x}(y) \\
& +51 G_{0,1-x}(y)+6 G_{1,1-x}(y)-15 G_{1-x, 0}(y)-15 G_{1-x, 1}(y)-9 G_{1-x, 1-x}(y)-15 G_{0,0}(x) \\
& \left.-3 G_{0,1}(x)+31 G_{1,0}(x)-41 G_{1,1}(x)-15 G_{0,0}(y)+51 G_{0,1}(y)-4 G_{1,0}(y)+20 G_{1,1}(y)\right) \\
& +\frac{1}{24} \zeta_{4}\left(183 G_{1-x}(y)-1467 G_{0}(x)+953 G_{1}(x)-1467 G_{0}(y)-130 G_{1}(y)\right) \\
& -13 \zeta_{5}-\frac{7}{3} \zeta_{2} \zeta_{3} \\
& g_{83}^{(6)}=162 G_{0,0,0}(x) G_{0,0,0}(y)-\frac{189}{2} G_{0,1,0}(x) G_{0,0,0}(y)-\frac{315}{2} G_{1,0,0}(x) G_{0,0,0}(y) \\
& +\frac{153}{2} G_{1,1,0}(x) G_{0,0,0}(y)-23 G_{0,0,1}(y) G_{0,1,0}(x)+\frac{259}{2} G_{0,0,1-x}(y) G_{0,1,0}(x) \\
& +19 G_{0,0,0}(x) G_{0,1,0}(y)-11 G_{0,1,0}(x) G_{0,1,0}(y)-14 G_{0,1,0}(x) G_{0,1,1}(y) \\
& +19 G_{0,1,0}(x) G_{0,1,1-x}(y)-\frac{137}{2} G_{0,0,0}(x) G_{0,1-x, 0}(y)+\frac{55}{2} G_{0,1,0}(x) G_{0,1-x, 0}(y) \\
& +15 G_{0,1,0}(x) G_{0,1-x, 1}(y)-\frac{79}{2} G_{0,1,0}(x) G_{0,1-x, 1-x}(y)+\frac{259}{2} G_{0,0,1-x}(y) G_{1,0,0}(x) \\
& -19 G_{0,1,0}(y) G_{1,0,0}(x)+19 G_{0,1,1-x}(y) G_{1,0,0}(x)+\frac{129}{2} G_{0,1-x, 0}(y) G_{1,0,0}(x) \\
& -\frac{79}{2} G_{0,1-x, 1-x}(y) G_{1,0,0}(x)+15 G_{0,0,0}(x) G_{1,0,0}(y)-9 G_{0,1,0}(x) G_{1,0,0}(y) \\
& -15 G_{1,0,0}(x) G_{1,0,0}(y)+4 G_{0,1,0}(x) G_{1,0,1}(y)+5 G_{0,1,0}(x) G_{1,0,1-x}(y) \\
& +5 G_{1,0,0}(x) G_{1,0,1-x}(y)-6 G_{0,1,0}(x) G_{1,0,-x}(y)+23 G_{0,0,1}(y) G_{1,1,0}(x) \\
& -\frac{125}{2} G_{0,0,1-x}(y) G_{1,1,0}(x)+11 G_{0,1,0}(y) G_{1,1,0}(x)+14 G_{0,1,1}(y) G_{1,1,0}(x)
\end{aligned}
$$


$-7 G_{0,1,1-x}(y) G_{1,1,0}(x)-\frac{39}{2} G_{0,1-x, 0}(y) G_{1,1,0}(x)-15 G_{0,1-x, 1}(y) G_{1,1,0}(x)$

$+\frac{21}{2} G_{0,1-x, 1-x}(y) G_{1,1,0}(x)+9 G_{1,0,0}(y) G_{1,1,0}(x)-4 G_{1,0,1}(y) G_{1,1,0}(x)$

$-7 G_{1,0,1-x}(y) G_{1,1,0}(x)-6 G_{1,0,-x}(y) G_{1,1,0}(x)+20 G_{0,0,0}(x) G_{1,1,0}(y)$

$-8 G_{0,1,0}(x) G_{1,1,0}(y)-20 G_{1,0,0}(x) G_{1,1,0}(y)+8 G_{1,1,0}(x) G_{1,1,0}(y)$

$-16 G_{0,1,0}(x) G_{1,1,1}(y)+16 G_{1,1,0}(x) G_{1,1,1}(y)+20 G_{0,1,0}(x) G_{1,1,1-x}(y)$

$+20 G_{1,0,0}(x) G_{1,1,1-x}(y)-4 G_{1,1,0}(x) G_{1,1,1-x}(y)-19 G_{0,0,0}(x) G_{1,1-x, 0}(y)$

$+13 G_{0,1,0}(x) G_{1,1-x, 0}(y)+15 G_{1,0,0}(x) G_{1,1-x, 0}(y)-7 G_{1,1,0}(x) G_{1,1-x, 0}(y)$

$+6 G_{0,1,0}(x) G_{1,1-x, 1}(y)-6 G_{1,1,0}(x) G_{1,1-x, 1}(y)-5 G_{0,1,0}(x) G_{1,1-x, 1-x}(y)$

$-5 G_{1,0,0}(x) G_{1,1-x, 1-x}(y)+3 G_{1,1,0}(x) G_{1,1-x, 1-x}(y)-6 G_{0,1,0}(x) G_{1,1-x,-x}(y)$

$-6 G_{1,1,0}(x) G_{1,1-x,-x}(y)-\frac{57}{2} G_{0,0,0}(x) G_{1-x, 0,0}(y)+\frac{9}{2} G_{0,1,0}(x) G_{1-x, 0,0}(y)$

$+\frac{51}{2} G_{1,0,0}(x) G_{1-x, 0,0}(y)+\frac{3}{2} G_{1,1,0}(x) G_{1-x, 0,0}(y)+3 G_{0,1,0}(x) G_{1-x, 0,1}(y)$

$-3 G_{1,1,0}(x) G_{1-x, 0,1}(y)-\frac{63}{2} G_{0,1,0}(x) G_{1-x, 0,1-x}(y)-\frac{63}{2} G_{1,0,0}(x) G_{1-x, 0,1-x}(y)$

$+\frac{5}{2} G_{1,1,0}(x) G_{1-x, 0,1-x}(y)+6 G_{0,1,0}(x) G_{1-x, 0,-x}(y)+6 G_{1,1,0}(x) G_{1-x, 0,-x}(y)$

$-15 G_{0,0,0}(x) G_{1-x, 1,0}(y)+7 G_{0,1,0}(x) G_{1-x, 1,0}(y)+15 G_{1,0,0}(x) G_{1-x, 1,0}(y)$

$-7 G_{1,1,0}(x) G_{1-x, 1,0}(y)+12 G_{0,1,0}(x) G_{1-x, 1,1}(y)-12 G_{1,1,0}(x) G_{1-x, 1,1}(y)$

$-15 G_{0,1,0}(x) G_{1-x, 1,1-x}(y)-15 G_{1,0,0}(x) G_{1-x, 1,1-x}(y)+5 G_{1,1,0}(x) G_{1-x, 1,1-x}(y)$

$+\frac{19}{2} G_{0,0,0}(x) G_{1-x, 1-x, 0}(y)-\frac{3}{2} G_{0,1,0}(x) G_{1-x, 1-x, 0}(y)-\frac{17}{2} G_{1,0,0}(x) G_{1-x, 1-x, 0}(y)$

$-\frac{1}{2} G_{1,1,0}(x) G_{1-x, 1-x, 0}(y)-3 G_{0,1,0}(x) G_{1-x, 1-x, 1}(y)+3 G_{1,1,0}(x) G_{1-x, 1-x, 1}(y)$

$+\frac{5}{2} G_{0,1,0}(x) G_{1-x, 1-x, 1-x}(y)+\frac{5}{2} G_{1,0,0}(x) G_{1-x, 1-x, 1-x}(y)+\frac{1}{2} G_{1,1,0}(x) G_{1-x, 1-x, 1-x}(y)$

$+162 G_{0,0}(y) G_{0,0,0,0}(x)+19 G_{1,0}(y) G_{0,0,0,0}(x)-\frac{65}{2} G_{1-x, 0}(y) G_{0,0,0,0}(x)$

$+162 G_{0,0}(x) G_{0,0,0,0}(y)-\frac{189}{2} G_{1,0}(x) G_{0,0,0,0}(y)+\frac{359}{2} G_{1,0}(x) G_{0,0,0,1-x}(y)$

$-\frac{189}{2} G_{0,0}(y) G_{0,0,1,0}(x)-19 G_{0,1}(y) G_{0,0,1,0}(x)+\frac{137}{2} G_{0,1-x}(y) G_{0,0,1,0}(x)$

- $15 G_{1,0}(y) G_{0,0,1,0}(x)-20 G_{1,1}(y) G_{0,0,1,0}(x)+13 G_{1,1-x}(y) G_{0,0,1,0}(x)$

$+\frac{21}{2} G_{1-x, 0}(y) G_{0,0,1,0}(x)+15 G_{1-x, 1}(y) G_{0,0,1,0}(x)-\frac{19}{2} G_{1-x, 1-x}(y) G_{0,0,1,0}(x)$

$+23 G_{0,0}(x) G_{0,0,1,0}(y)-23 G_{1,0}(x) G_{0,0,1,0}(y)+23 G_{1,0}(x) G_{0,0,1,1-x}(y)$

$-\frac{259}{2} G_{0,0}(x) G_{0,0,1-x, 0}(y)+\frac{169}{2} G_{1,0}(x) G_{0,0,1-x, 0}(y)-\frac{203}{2} G_{1,0}(x) G_{0,0,1-x, 1-x}(y)$

$-\frac{315}{2} G_{0,0}(y) G_{0,1,0,0}(x)-19 G_{0,1}(y) G_{0,1,0,0}(x)+\frac{167}{2} G_{0,1-x}(y) G_{0,1,0,0}(x)$

$-15 G_{1,0}(y) G_{0,1,0,0}(x)-20 G_{1,1}(y) G_{0,1,0,0}(x)+25 G_{1,1-x}(y) G_{0,1,0,0}(x)$

$+\frac{51}{2} G_{1-x, 0}(y) G_{0,1,0,0}(x)+15 G_{1-x, 1}(y) G_{0,1,0,0}(x)-\frac{25}{2} G_{1-x, 1-x}(y) G_{0,1,0,0}(x)$

$+15 G_{0,0}(x) G_{0,1,0,0}(y)-15 G_{1,0}(x) G_{0,1,0,0}(y)+9 G_{1,0}(x) G_{0,1,0,1-x}(y)$

$+\frac{153}{2} G_{0,0}(y) G_{0,1,1,0}(x)+7 G_{0,1}(y) G_{0,1,1,0}(x)-\frac{11}{2} G_{0,1-x}(y) G_{0,1,1,0}(x)$ 


$$
\begin{aligned}
& +9 G_{1,0}(y) G_{0,1,1,0}(x)+4 G_{1,1}(y) G_{0,1,1,0}(x)-11 G_{1,1-x}(y) G_{0,1,1,0}(x) \\
& +\frac{3}{2} G_{1-x, 0}(y) G_{0,1,1,0}(x)-5 G_{1-x, 1}(y) G_{0,1,1,0}(x)-\frac{3}{2} G_{1-x, 1-x}(y) G_{0,1,1,0}(x) \\
& +14 G_{0,0}(x) G_{0,1,1,0}(y)-14 G_{1,0}(x) G_{0,1,1,0}(y)+14 G_{1,0}(x) G_{0,1,1,1-x}(y) \\
& -19 G_{0,0}(x) G_{0,1,1-x, 0}(y)+11 G_{1,0}(x) G_{0,1,1-x, 0}(y)-5 G_{1,0}(x) G_{0,1,1-x, 1-x}(y) \\
& -\frac{129}{2} G_{0,0}(x) G_{0,1-x, 0,0}(y)+\frac{63}{2} G_{1,0}(x) G_{0,1-x, 0,0}(y)-\frac{151}{2} G_{1,0}(x) G_{0,1-x, 0,1-x}(y) \\
& -15 G_{0,0}(x) G_{0,1-x, 1,0}(y)+15 G_{1,0}(x) G_{0,1-x, 1,0}(y)-15 G_{1,0}(x) G_{0,1-x, 1,1-x}(y) \\
& +\frac{79}{2} G_{0,0}(x) G_{0,1-x, 1-x, 0}(y)-\frac{45}{2} G_{1,0}(x) G_{0,1-x, 1-x, 0}(y)+\frac{35}{2} G_{1,0}(x) G_{0,1-x, 1-x, 1-x}(y) \\
& -\frac{399}{2} G_{0,0}(y) G_{1,0,0,0}(x)+\frac{137}{2} G_{0,1-x}(y) G_{1,0,0,0}(x)-19 G_{1,0}(y) G_{1,0,0,0}(x) \\
& +19 G_{1,1-x}(y) G_{1,0,0,0}(x)+\frac{91}{2} G_{1-x, 0}(y) G_{1,0,0,0}(x)-\frac{19}{2} G_{1-x, 1-x}(y) G_{1,0,0,0}(x) \\
& +9 G_{0,0}(x) G_{1,0,0,0}(y)-9 G_{1,0}(x) G_{1,0,0,0}(y)+3 G_{1,0}(x) G_{1,0,0,1-x}(y) \\
& +\frac{225}{2} G_{0,0}(y) G_{1,0,1,0}(x)+19 G_{0,1}(y) G_{1,0,1,0}(x)-\frac{55}{2} G_{0,1-x}(y) G_{1,0,1,0}(x) \\
& +13 G_{1,0}(y) G_{1,0,1,0}(x)+20 G_{1,1}(y) G_{1,0,1,0}(x)-13 G_{1,1-x}(y) G_{1,0,1,0}(x) \\
& -\frac{33}{2} G_{1-x, 0}(y) G_{1,0,1,0}(x)-15 G_{1-x, 1}(y) G_{1,0,1,0}(x)+\frac{5}{2} G_{1-x, 1-x}(y) G_{1,0,1,0}(x) \\
& -4 G_{0,0}(x) G_{1,0,1,0}(y)+4 G_{1,0}(x) G_{1,0,1,0}(y)-4 G_{1,0}(x) G_{1,0,1,1-x}(y) \\
& -5 G_{0,0}(x) G_{1,0,1-x, 0}(y)+5 G_{1,0}(x) G_{1,0,1-x, 0}(y)-G_{1,0}(x) G_{1,0,1-x, 1-x}(y) \\
& +6 G_{1,0}(x) G_{1,0,-x, 0}(y)-6 G_{1,0}(x) G_{1,0,-x, 1-x}(y)+\frac{327}{2} G_{0,0}(y) G_{1,1,0,0}(x) \\
& +19 G_{0,1}(y) G_{1,1,0,0}(x)-\frac{129}{2} G_{0,1-x}(y) G_{1,1,0,0}(x)+15 G_{1,0}(y) G_{1,1,0,0}(x) \\
& +20 G_{1,1}(y) G_{1,1,0,0}(x)-15 G_{1,1-x}(y) G_{1,1,0,0}(x)-\frac{55}{2} G_{1-x, 0}(y) G_{1,1,0,0}(x) \\
& -15 G_{1-x, 1}(y) G_{1,1,0,0}(x)+\frac{19}{2} G_{1-x, 1-x}(y) G_{1,1,0,0}(x)+12 G_{0,0}(x) G_{1,1,0,0}(y) \\
& -12 G_{1,0}(x) G_{1,1,0,0}(y)+8 G_{1,0}(x) G_{1,1,0,1-x}(y)-\frac{129}{2} G_{0,0}(y) G_{1,1,1,0}(x) \\
& -7 G_{0,1}(y) G_{1,1,1,0}(x)+\frac{27}{2} G_{0,1-x}(y) G_{1,1,1,0}(x)-7 G_{1,0}(y) G_{1,1,1,0}(x) \\
& -4 G_{1,1}(y) G_{1,1,1,0}(x)+3 G_{1,1-x}(y) G_{1,1,1,0}(x)-\frac{11}{2} G_{1-x, 0}(y) G_{1,1,1,0}(x) \\
& +5 G_{1-x, 1}(y) G_{1,1,1,0}(x)+\frac{3}{2} G_{1-x, 1-x}(y) G_{1,1,1,0}(x)+16 G_{0,0}(x) G_{1,1,1,0}(y) \\
& -16 G_{1,0}(x) G_{1,1,1,0}(y)+16 G_{1,0}(x) G_{1,1,1,1-x}(y)-20 G_{0,0}(x) G_{1,1,1-x, 0}(y) \\
& +8 G_{1,0}(x) G_{1,1,1-x, 0}(y)-4 G_{1,0}(x) G_{1,1,1-x, 1-x}(y)-15 G_{0,0}(x) G_{1,1-x, 0,0}(y) \\
& +9 G_{1,0}(x) G_{1,1-x, 0,0}(y)-5 G_{1,0}(x) G_{1,1-x, 0,1-x}(y)-6 G_{0,0}(x) G_{1,1-x, 1,0}(y) \\
& +6 G_{1,0}(x) G_{1,1-x, 1,0}(y)-6 G_{1,0}(x) G_{1,1-x, 1,1-x}(y)+5 G_{0,0}(x) G_{1,1-x, 1-x, 0}(y) \\
& -3 G_{1,0}(x) G_{1,1-x, 1-x, 0}(y)+G_{1,0}(x) G_{1,1-x, 1-x, 1-x}(y)+6 G_{1,0}(x) G_{1,1-x,-x, 0}(y) \\
& -6 G_{1,0}(x) G_{1,1-x,-x, 1-x}(y)-\frac{45}{2} G_{0,0}(x) G_{1-x, 0,0,0}(y)+\frac{9}{2} G_{1,0}(x) G_{1-x, 0,0,0}(y) \\
& -\frac{79}{2} G_{1,0}(x) G_{1-x, 0,0,1-x}(y)-3 G_{0,0}(x) G_{1-x, 0,1,0}(y)+3 G_{1,0}(x) G_{1-x, 0,1,0}(y)
\end{aligned}
$$


$-3 G_{1,0}(x) G_{1-x, 0,1,1-x}(y)+\frac{63}{2} G_{0,0}(x) G_{1-x, 0,1-x, 0}(y)-\frac{25}{2} G_{1,0}(x) G_{1-x, 0,1-x, 0}(y)$

$+\frac{51}{2} G_{1,0}(x) G_{1-x, 0,1-x, 1-x}(y)-6 G_{1,0}(x) G_{1-x, 0,-x, 0}(y)+6 G_{1,0}(x) G_{1-x, 0,-x, 1-x}(y)$

$-9 G_{0,0}(x) G_{1-x, 1,0,0}(y)+9 G_{1,0}(x) G_{1-x, 1,0,0}(y)-5 G_{1,0}(x) G_{1-x, 1,0,1-x}(y)$

$-12 G_{0,0}(x) G_{1-x, 1,1,0}(y)+12 G_{1,0}(x) G_{1-x, 1,1,0}(y)-12 G_{1,0}(x) G_{1-x, 1,1,1-x}(y)$

$+15 G_{0,0}(x) G_{1-x, 1,1-x, 0}(y)-7 G_{1,0}(x) G_{1-x, 1,1-x, 0}(y)+3 G_{1,0}(x) G_{1-x, 1,1-x, 1-x}(y)$

$+\frac{15}{2} G_{0,0}(x) G_{1-x, 1-x, 0,0}(y)-\frac{3}{2} G_{1,0}(x) G_{1-x, 1-x, 0,0}(y)+\frac{15}{2} G_{1,0}(x) G_{1-x, 1-x, 0,1-x}(y)$

$+3 G_{0,0}(x) G_{1-x, 1-x, 1,0}(y)-3 G_{1,0}(x) G_{1-x, 1-x, 1,0}(y)+3 G_{1,0}(x) G_{1-x, 1-x, 1,1-x}(y)$

$-\frac{5}{2} G_{0,0}(x) G_{1-x, 1-x, 1-x, 0}(y)+\frac{1}{2} G_{1,0}(x) G_{1-x, 1-x, 1-x, 0}(y)+162 G_{0}(y) G_{0,0,0,0,0}(x)$

$-\frac{1}{2} G_{1,0}(x) G_{1-x, 1-x, 1-x, 1-x}(y)+162 G_{0}(x) G_{0,0,0,0,0}(y)-\frac{189}{2} G_{0}(y) G_{0,0,0,1,0}(x)$

$-13 G_{1}(y) G_{0,0,0,1,0}(x)+\frac{53}{2} G_{1-x}(y) G_{0,0,0,1,0}(x)-\frac{359}{2} G_{0}(x) G_{0,0,0,1-x, 0}(y)$

$-\frac{315}{2} G_{0}(y) G_{0,0,1,0,0}(x)-25 G_{1}(y) G_{0,0,1,0,0}(x)+\frac{95}{2} G_{1-x}(y) G_{0,0,1,0,0}(x)$

$+\frac{153}{2} G_{0}(y) G_{0,0,1,1,0}(x)+15 G_{1}(y) G_{0,0,1,1,0}(x)-\frac{3}{2} G_{1-x}(y) G_{0,0,1,1,0}(x)$

$-23 G_{0}(x) G_{0,0,1,1-x, 0}(y)-\frac{213}{2} G_{0}(x) G_{0,0,1-x, 0,0}(y)+\frac{203}{2} G_{0}(x) G_{0,0,1-x, 1-x, 0}(y)$

$-\frac{399}{2} G_{0}(y) G_{0,1,0,0,0}(x)-19 G_{1}(y) G_{0,1,0,0,0}(x)+\frac{95}{2} G_{1-x}(y) G_{0,1,0,0,0}(x)$

$+\frac{225}{2} G_{0}(y) G_{0,1,0,1,0}(x)+13 G_{1}(y) G_{0,1,0,1,0}(x)-\frac{11}{2} G_{1-x}(y) G_{0,1,0,1,0}(x)$

$-9 G_{0}(x) G_{0,1,0,1-x, 0}(y)+\frac{327}{2} G_{0}(y) G_{0,1,1,0,0}(x)+15 G_{1}(y) G_{0,1,1,0,0}(x)$

$-\frac{57}{2} G_{1-x}(y) G_{0,1,1,0,0}(x)-\frac{129}{2} G_{0}(y) G_{0,1,1,1,0}(x)-7 G_{1}(y) G_{0,1,1,1,0}(x)$

$-\frac{13}{2} G_{1-x}(y) G_{0,1,1,1,0}(x)-14 G_{0}(x) G_{0,1,1,1-x, 0}(y)-15 G_{0}(x) G_{0,1,1-x, 0,0}(y)$

$+5 G_{0}(x) G_{0,1,1-x, 1-x, 0}(y)-\frac{99}{2} G_{0}(x) G_{0,1-x, 0,0,0}(y)+\frac{151}{2} G_{0}(x) G_{0,1-x, 0,1-x, 0}(y)$

$+15 G_{0}(x) G_{0,1-x, 1,1-x, 0}(y)+\frac{69}{2} G_{0}(x) G_{0,1-x, 1-x, 0,0}(y)-\frac{35}{2} G_{0}(x) G_{0,1-x, 1-x, 1-x, 0}(y)$

$-\frac{455}{2} G_{0}(y) G_{1,0,0,0,0}(x)+\frac{65}{2} G_{1-x}(y) G_{1,0,0,0,0}(x)+\frac{273}{2} G_{0}(y) G_{1,0,0,1,0}(x)$

$+13 G_{1}(y) G_{1,0,0,1,0}(x)-\frac{43}{2} G_{1-x}(y) G_{1,0,0,1,0}(x)-3 G_{0}(x) G_{1,0,0,1-x, 0}(y)$

$+\frac{399}{2} G_{0}(y) G_{1,0,1,0,0}(x)+25 G_{1}(y) G_{1,0,1,0,0}(x)-\frac{85}{2} G_{1-x}(y) G_{1,0,1,0,0}(x)$

$-\frac{185}{2} G_{0}(y) G_{1,0,1,1,0}(x)-11 G_{1}(y) G_{1,0,1,1,0}(x)+\frac{27}{2} G_{1-x}(y) G_{1,0,1,1,0}(x)$

$+4 G_{0}(x) G_{1,0,1,1-x, 0}(y)-3 G_{0}(x) G_{1,0,1-x, 0,0}(y)+G_{0}(x) G_{1,0,1-x, 1-x, 0}(y)$

$+6 G_{0}(x) G_{1,0,-x, 1-x, 0}(y)+\frac{467}{2} G_{0}(y) G_{1,1,0,0,0}(x)+19 G_{1}(y) G_{1,1,0,0,0}(x)$

$-\frac{125}{2} G_{1-x}(y) G_{1,1,0,0,0}(x)-\frac{265}{2} G_{0}(y) G_{1,1,0,1,0}(x)-13 G_{1}(y) G_{1,1,0,1,0}(x)$

$+\frac{55}{2} G_{1-x}(y) G_{1,1,0,1,0}(x)-8 G_{0}(x) G_{1,1,0,1-x, 0}(y)-\frac{335}{2} G_{0}(y) G_{1,1,1,0,0}(x)$ 
$-15 G_{1}(y) G_{1,1,1,0,0}(x)+\frac{57}{2} G_{1-x}(y) G_{1,1,1,0,0}(x)+\frac{113}{2} G_{0}(y) G_{1,1,1,1,0}(x)$

$+3 G_{1}(y) G_{1,1,1,1,0}(x)+\frac{17}{2} G_{1-x}(y) G_{1,1,1,1,0}(x)-16 G_{0}(x) G_{1,1,1,1-x, 0}(y)$

$-12 G_{0}(x) G_{1,1,1-x, 0,0}(y)+4 G_{0}(x) G_{1,1,1-x, 1-x, 0}(y)-9 G_{0}(x) G_{1,1-x, 0,0,0}(y)$

$+5 G_{0}(x) G_{1,1-x, 0,1-x, 0}(y)+6 G_{0}(x) G_{1,1-x, 1,1-x, 0}(y)+3 G_{0}(x) G_{1,1-x, 1-x, 0,0}(y)$

$-G_{0}(x) G_{1,1-x, 1-x, 1-x, 0}(y)+6 G_{0}(x) G_{1,1-x,-x, 1-x, 0}(y)-\frac{27}{2} G_{0}(x) G_{1-x, 0,0,0,0}(y)$

$+\frac{79}{2} G_{0}(x) G_{1-x, 0,0,1-x, 0}(y)+3 G_{0}(x) G_{1-x, 0,1,1-x, 0}(y)+\frac{45}{2} G_{0}(x) G_{1-x, 0,1-x, 0,0}(y)$

$-\frac{51}{2} G_{0}(x) G_{1-x, 0,1-x, 1-x, 0}(y)-6 G_{0}(x) G_{1-x, 0,-x, 1-x, 0}(y)+5 G_{0}(x) G_{1-x, 1,0,1-x, 0}(y)$

$+12 G_{0}(x) G_{1-x, 1,1,1-x, 0}(y)+9 G_{0}(x) G_{1-x, 1,1-x, 0,0}(y)-3 G_{0}(x) G_{1-x, 1,1-x, 1-x, 0}(y)$

$+\frac{9}{2} G_{0}(x) G_{1-x, 1-x, 0,0,0}(y)-\frac{15}{2} G_{0}(x) G_{1-x, 1-x, 0,1-x, 0}(y)-3 G_{0}(x) G_{1-x, 1-x, 1,1-x, 0}(y)$

$-\frac{3}{2} G_{0}(x) G_{1-x, 1-x, 1-x, 0,0}(y)+\frac{1}{2} G_{0}(x) G_{1-x, 1-x, 1-x, 1-x, 0}(y)+162 G_{0,0,0,0,0,0}(x)$

$+162 G_{0,0,0,0,0,0}(y)-\frac{189}{2} G_{0,0,0,0,1,0}(x)-\frac{1571}{6} G_{0,0,0,0,1,0}(y)-\frac{315}{2} G_{0,0,0,1,0,0}(x)$

$-\frac{359}{2} G_{0,0,0,1,0,0}(y)+\frac{153}{2} G_{0,0,0,1,1,0}(x)-\frac{46}{3} G_{0,0,0,1,1,0}(y)+\frac{359}{2} G_{0,0,0,1-x, 1,0}(y)$

$-\frac{399}{2} G_{0,0,1,0,0,0}(x)-\frac{213}{2} G_{0,0,1,0,0,0}(y)+\frac{225}{2} G_{0,0,1,0,1,0}(x)+\frac{62}{3} G_{0,0,1,0,1,0}(y)$

$+\frac{327}{2} G_{0,0,1,1,0,0}(x)-28 G_{0,0,1,1,0,0}(y)-\frac{129}{2} G_{0,0,1,1,1,0}(x)-\frac{52}{3} G_{0,0,1,1,1,0}(y)$

$+23 G_{0,0,1,1-x, 1,0}(y)+\frac{247}{2} G_{0,0,1-x, 0,1,0}(y)+\frac{213}{2} G_{0,0,1-x, 1,0,0}(y)+28 G_{0,0,1-x, 1,1,0}(y)$

$-\frac{203}{2} G_{0,0,1-x, 1-x, 1,0}(y)-\frac{455}{2} G_{0,1,0,0,0,0}(x)-\frac{99}{2} G_{0,1,0,0,0,0}(y)+\frac{273}{2} G_{0,1,0,0,1,0}(x)$

$+\frac{44}{3} G_{0,1,0,0,1,0}(y)+\frac{399}{2} G_{0,1,0,1,0,0}(x)+14 G_{0,1,0,1,0,0}(y)-\frac{185}{2} G_{0,1,0,1,1,0}(x)$

$-\frac{16}{3} G_{0,1,0,1,1,0}(y)+9 G_{0,1,0,1-x, 1,0}(y)+\frac{467}{2} G_{0,1,1,0,0,0}(x)-30 G_{0,1,1,0,0,0}(y)$

$-\frac{265}{2} G_{0,1,1,0,1,0}(x)+\frac{38}{3} G_{0,1,1,0,1,0}(y)-\frac{335}{2} G_{0,1,1,1,0,0}(x)-22 G_{0,1,1,1,0,0}(y)$

$+\frac{113}{2} G_{0,1,1,1,1,0}(x)-\frac{70}{3} G_{0,1,1,1,1,0}(y)+14 G_{0,1,1,1-x, 1,0}(y)+9 G_{0,1,1-x, 0,1,0}(y)$

$+15 G_{0,1,1-x, 1,0,0}(y)+14 G_{0,1,1-x, 1,1,0}(y)-5 G_{0,1,1-x, 1-x, 1,0}(y)+\frac{187}{2} G_{0,1-x, 0,0,1,0}(y)$

$+\frac{105}{2} G_{0,1-x, 0,1,0,0}(y)+10 G_{0,1-x, 0,1,1,0}(y)-\frac{151}{2} G_{0,1-x, 0,1-x, 1,0}(y)+\frac{99}{2} G_{0,1-x, 1,0,0,0}(y)$

$-14 G_{0,1-x, 1,0,1,0}(y)+30 G_{0,1-x, 1,1,0,0}(y)+22 G_{0,1-x, 1,1,1,0}(y)-15 G_{0,1-x, 1,1-x, 1,0}(y)$

$-\frac{59}{2} G_{0,1-x, 1-x, 0,1,0}(y)-\frac{69}{2} G_{0,1-x, 1-x, 1,0,0}(y)-22 G_{0,1-x, 1-x, 1,1,0}(y)+\frac{2}{3} G_{1,0,0,1,1,0}(y)$

$+\frac{35}{2} G_{0,1-x, 1-x, 1-x, 1,0}(y)-\frac{1477}{6} G_{1,0,0,0,0,0}(x)-\frac{27}{2} G_{1,0,0,0,0,0}(y)+\frac{305}{2} G_{1,0,0,0,1,0}(x)$

$+\frac{26}{3} G_{1,0,0,0,1,0}(y)+\frac{447}{2} G_{1,0,0,1,0,0}(x)+8 G_{1,0,0,1,0,0}(y)-\frac{667}{6} G_{1,0,0,1,1,0}(x)$

$+3 G_{1,0,0,1-x, 1,0}(y)+\frac{523}{2} G_{1,0,1,0,0,0}(x)+6 G_{1,0,1,0,0,0}(y)-\frac{931}{6} G_{1,0,1,0,1,0}(x)$

$-\frac{10}{3} G_{1,0,1,0,1,0}(y)-\frac{1189}{6} G_{1,0,1,1,0,0}(x)+2 G_{1,0,1,1,0,0}(y)+\frac{153}{2} G_{1,0,1,1,1,0}(x)$ 


$$
\begin{aligned}
& +\frac{8}{3} G_{1,0,1,1,1,0}(y)-4 G_{1,0,1,1-x, 1,0}(y)-G_{1,0,1-x, 0,1,0}(y)+3 G_{1,0,1-x, 1,0,0}(y) \\
& +4 G_{1,0,1-x, 1,1,0}(y)-G_{1,0,1-x, 1-x, 1,0}(y)-6 G_{1,0,-x, 0,1,0}(y)-6 G_{1,0,-x, 1-x, 1,0}(y) \\
& +\frac{1705}{6} G_{1,1,0,0,0,0}(x)-18 G_{1,1,0,0,0,0}(y)-\frac{1019}{6} G_{1,1,0,0,1,0}(x)+\frac{32}{3} G_{1,1,0,0,1,0}(y) \\
& -\frac{1445}{6} G_{1,1,0,1,0,0}(x)+8 G_{1,1,0,1,0,0}(y)+\frac{667}{6} G_{1,1,0,1,1,0}(x)+\frac{8}{3} G_{1,1,0,1,1,0}(y) \\
& +8 G_{1,1,0,1-x, 1,0}(y)-\frac{1561}{6} G_{1,1,1,0,0,0}(x)-24 G_{1,1,1,0,0,0}(y)+\frac{289}{2} G_{1,1,1,0,1,0}(x) \\
& +\frac{32}{3} G_{1,1,1,0,1,0}(y)+\frac{1021}{6} G_{1,1,1,1,0,0}(x)-32 G_{1,1,1,1,0,0}(y)-\frac{307}{6} G_{1,1,1,1,1,0}(x) \\
& -\frac{128}{3} G_{1,1,1,1,1,0}(y)+16 G_{1,1,1,1-x, 1,0}(y)+8 G_{1,1,1-x, 0,1,0}(y)+12 G_{1,1,1-x, 1,0,0}(y) \\
& +16 G_{1,1,1-x, 1,1,0}(y)-4 G_{1,1,1-x, 1-x, 1,0}(y)+3 G_{1,1-x, 0,0,1,0}(y)+3 G_{1,1-x, 0,1,0,0}(y) \\
& +4 G_{1,1-x, 0,1,1,0}(y)-5 G_{1,1-x, 0,1-x, 1,0}(y)+9 G_{1,1-x, 1,0,0,0}(y)-4 G_{1,1-x, 1,0,1,0}(y) \\
& +12 G_{1,1-x, 1,1,0,0}(y)+16 G_{1,1-x, 1,1,1,0}(y)-6 G_{1,1-x, 1,1-x, 1,0}(y)-G_{1,1-x, 1-x, 0,1,0}(y) \\
& -3 G_{1,1-x, 1-x, 1,0,0}(y)-4 G_{1,1-x, 1-x, 1,1,0}(y)+G_{1,1-x, 1-x, 1-x, 1,0}(y)-6 G_{1,1-x,-x, 0,1,0}(y) \\
& -6 G_{1,1-x,-x, 1-x, 1,0}(y)+\frac{103}{2} G_{1-x, 0,0,0,1,0}(y)+\frac{57}{2} G_{1-x, 0,0,1,0,0}(y)+2 G_{1-x, 0,0,1,1,0}(y) \\
& -\frac{79}{2} G_{1-x, 0,0,1-x, 1,0}(y)+\frac{27}{2} G_{1-x, 0,1,0,0,0}(y)-4 G_{1-x, 0,1,0,1,0}(y)-6 G_{1-x, 0,1,1,1,0}(y) \\
& -3 G_{1-x, 0,1,1-x, 1,0}(y)-\frac{71}{2} G_{1-x, 0,1-x, 0,1,0}(y)-\frac{45}{2} G_{1-x, 0,1-x, 1,0,0}(y) \\
& -6 G_{1-x, 0,1-x, 1,1,0}(y)+\frac{51}{2} G_{1-x, 0,1-x, 1-x, 1,0}(y)+6 G_{1-x, 0,-x, 0,1,0}(y) \\
& +6 G_{1-x, 0,-x, 1-x, 1,0}(y)+\frac{27}{2} G_{1-x, 1,0,0,0,0}(y)-8 G_{1-x, 1,0,0,1,0}(y)-6 G_{1-x, 1,0,1,0,0}(y) \\
& -2 G_{1-x, 1,0,1,1,0}(y)-5 G_{1-x, 1,0,1-x, 1,0}(y)+18 G_{1-x, 1,1,0,0,0}(y)-8 G_{1-x, 1,1,0,1,0}(y) \\
& +24 G_{1-x, 1,1,1,0,0}(y)+32 G_{1-x, 1,1,1,1,0}(y)-12 G_{1-x, 1,1,1-x, 1,0}(y)-5 G_{1-x, 1,1-x, 0,1,0}(y) \\
& -9 G_{1-x, 1,1-x, 1,0,0}(y)-12 G_{1-x, 1,1-x, 1,1,0}(y) \\
& +3 G_{1-x, 1,1-x, 1-x, 1,0}(y)-\frac{19}{2} G_{1-x, 1-x, 0,0,1,0}(y)-\frac{9}{2} G_{1-x, 1-x, 0,1,0,0}(y) \\
& +\frac{15}{2} G_{1-x, 1-x, 0,1-x, 1,0}(y)-\frac{9}{2} G_{1-x, 1-x, 1,0,0,0}(y)+2 G_{1-x, 1-x, 1,0,1,0}(y) \\
& -6 G_{1-x, 1-x, 1,1,0,0}(y)-8 G_{1-x, 1-x, 1,1,1,0}(y)+3 G_{1-x, 1-x, 1,1-x, 1,0}(y) \\
& +\frac{3}{2} G_{1-x, 1-x, 1-x, 0,1,0}(y)+\frac{3}{2} G_{1-x, 1-x, 1-x, 1,0,0}(y) \\
& +2 G_{1-x, 1-x, 1-x, 1,1,0}(y)-\frac{1}{2} G_{1-x, 1-x, 1-x, 1-x, 1,0}(y) \\
& +\frac{1}{6} \zeta_{2}\left(729 G_{0,0}(x) G_{0,0}(y)-459 G_{0,1}(x) G_{0,0}(y)-729 G_{1,0}(x) G_{0,0}(y)\right. \\
& +387 G_{1,1}(x) G_{0,0}(y)+30 G_{0,0}(x) G_{0,1}(y)-42 G_{0,1}(x) G_{0,1}(y)-147 G_{0,0}(x) G_{0,1-x}(y) \\
& +33 G_{0,1}(x) G_{0,1-x}(y)-30 G_{0,1}(y) G_{1,0}(x)+183 G_{0,1-x}(y) G_{1,0}(x)+90 G_{0,0}(x) G_{1,0}(y) \\
& -54 G_{0,1}(x) G_{1,0}(y)-78 G_{1,0}(x) G_{1,0}(y)+42 G_{0,1}(y) G_{1,1}(x)-81 G_{0,1-x}(y) G_{1,1}(x) \\
& +42 G_{1,0}(y) G_{1,1}(x)+24 G_{0,0}(x) G_{1,1}(y)-24 G_{0,1}(x) G_{1,1}(y)-24 G_{1,0}(x) G_{1,1}(y) \\
& +24 G_{1,1}(x) G_{1,1}(y)+6 G_{0,0}(x) G_{1,1-x}(y)+66 G_{0,1}(x) G_{1,1-x}(y) \\
& +78 G_{1,0}(x) G_{1,1-x}(y)-18 G_{1,1}(x) G_{1,1-x}(y)-117 G_{0,0}(x) G_{1-x, 0}(y)
\end{aligned}
$$




$$
\begin{aligned}
& -9 G_{0,1}(x) G_{1-x, 0}(y)+117 G_{1,0}(x) G_{1-x, 0}(y)+33 G_{1,1}(x) G_{1-x, 0}(y) \\
& -18 G_{0,0}(x) G_{1-x, 1}(y)+30 G_{0,1}(x) G_{1-x, 1}(y)+18 G_{1,0}(x) G_{1-x, 1}(y) \\
& -30 G_{1,1}(x) G_{1-x, 1}(y)-3 G_{0,0}(x) G_{1-x, 1-x}(y)+9 G_{0,1}(x) G_{1-x, 1-x}(y) \\
& -21 G_{1,0}(x) G_{1-x, 1-x}(y)-9 G_{1,1}(x) G_{1-x, 1-x}(y)+729 G_{0}(y) G_{0,0,0}(x) \\
& -6 G_{1}(y) G_{0,0,0}(x)+33 G_{1-x}(y) G_{0,0,0}(x)+729 G_{0}(x) G_{0,0,0}(y) \\
& \text { - } 459 G_{1}(x) G_{0,0,0}(y)-459 G_{0}(y) G_{0,0,1}(x)-90 G_{1}(y) G_{0,0,1}(x) \\
& +9 G_{1-x}(y) G_{0,0,1}(x)+138 G_{0}(x) G_{0,0,1}(y)-138 G_{1}(x) G_{0,0,1}(y) \\
& \text { - } 609 G_{0}(x) G_{0,0,1-x}(y)+375 G_{1}(x) G_{0,0,1-x}(y)-729 G_{0}(y) G_{0,1,0}(x) \\
& -78 G_{1}(y) G_{0,1,0}(x)+123 G_{1-x}(y) G_{0,1,0}(x)+66 G_{0}(x) G_{0,1,0}(y) \\
& -66 G_{1}(x) G_{0,1,0}(y)+387 G_{0}(y) G_{0,1,1}(x)+42 G_{1}(y) G_{0,1,1}(x) \\
& +39 G_{1-x}(y) G_{0,1,1}(x)+84 G_{0}(x) G_{0,1,1}(y)-84 G_{1}(x) G_{0,1,1}(y) \\
& -30 G_{0}(x) G_{0,1,1-x}(y)+42 G_{1}(x) G_{0,1,1-x}(y)-255 G_{0}(x) G_{0,1-x, 0}(y) \\
& +117 G_{1}(x) G_{0,1-x, 0}(y)-90 G_{0}(x) G_{0,1-x, 1}(y)+90 G_{1}(x) G_{0,1-x, 1}(y) \\
& +105 G_{0}(x) G_{0,1-x, 1-x}(y)-63 G_{1}(x) G_{0,1-x, 1-x}(y)-909 G_{0}(y) G_{1,0,0}(x) \\
& +6 G_{1}(y) G_{1,0,0}(x)+123 G_{1-x}(y) G_{1,0,0}(x)+54 G_{0}(x) G_{1,0,0}(y) \\
& -54 G_{1}(x) G_{1,0,0}(y)+555 G_{0}(y) G_{1,0,1}(x)+66 G_{1}(y) G_{1,0,1}(x) \\
& -81 G_{1-x}(y) G_{1,0,1}(x)-24 G_{0}(x) G_{1,0,1}(y)+24 G_{1}(x) G_{1,0,1}(y) \\
& -6 G_{0}(x) G_{1,0,1-x}(y)+42 G_{1}(x) G_{1,0,1-x}(y)+36 G_{1}(x) G_{1,0,-x}(y) \\
& +777 G_{0}(y) G_{1,1,0}(x)+78 G_{1}(y) G_{1,1,0}(x)-159 G_{1-x}(y) G_{1,1,0}(x) \\
& +48 G_{0}(x) G_{1,1,0}(y)-48 G_{1}(x) G_{1,1,0}(y)-339 G_{0}(y) G_{1,1,1}(x) \\
& \text { - } 18 G_{1}(y) G_{1,1,1}(x)-51 G_{1-x}(y) G_{1,1,1}(x)+96 G_{0}(x) G_{1,1,1}(y) \\
& \text { - } 96 G_{1}(x) G_{1,1,1}(y)-24 G_{0}(x) G_{1,1,1-x}(y)+24 G_{1}(x) G_{1,1,1-x}(y) \\
& -78 G_{0}(x) G_{1,1-x, 0}(y)+42 G_{1}(x) G_{1,1-x, 0}(y)-36 G_{0}(x) G_{1,1-x, 1}(y) \\
& +36 G_{1}(x) G_{1,1-x, 1}(y)+6 G_{0}(x) G_{1,1-x, 1-x}(y)-18 G_{1}(x) G_{1,1-x, 1-x}(y) \\
& +36 G_{1}(x) G_{1,1-x,-x}(y)-81 G_{0}(x) G_{1-x, 0,0}(y)-9 G_{1}(x) G_{1-x, 0,0}(y) \\
& -18 G_{0}(x) G_{1-x, 0,1}(y)+18 G_{1}(x) G_{1-x, 0,1}(y)+153 G_{0}(x) G_{1-x, 0,1-x}(y) \\
& -15 G_{1}(x) G_{1-x, 0,1-x}(y)-36 G_{1}(x) G_{1-x, 0,-x}(y)-42 G_{0}(x) G_{1-x, 1,0}(y) \\
& +42 G_{1}(x) G_{1-x, 1,0}(y)-72 G_{0}(x) G_{1-x, 1,1}(y)+72 G_{1}(x) G_{1-x, 1,1}(y) \\
& +18 G_{0}(x) G_{1-x, 1,1-x}(y)-30 G_{1}(x) G_{1-x, 1,1-x}(y)+27 G_{0}(x) G_{1-x, 1-x, 0}(y) \\
& +3 G_{1}(x) G_{1-x, 1-x, 0}(y)+18 G_{0}(x) G_{1-x, 1-x, 1}(y)-18 G_{1}(x) G_{1-x, 1-x, 1}(y) \\
& -3 G_{0}(x) G_{1-x, 1-x, 1-x}(y)-3 G_{1}(x) G_{1-x, 1-x, 1-x}(y)+729 G_{0,0,0,0}(x) \\
& +729 G_{0,0,0,0}(y)-459 G_{0,0,0,1}(x)+92 G_{0,0,0,1}(y)-1077 G_{0,0,0,1-x}(y) \\
& -729 G_{0,0,1,0}(x)+387 G_{0,0,1,1}(x)+104 G_{0,0,1,1}(y)-138 G_{0,0,1,1-x}(y) \\
& -507 G_{0,0,1-x, 0}(y)-168 G_{0,0,1-x, 1}(y)+609 G_{0,0,1-x, 1-x}(y)-909 G_{0,1,0,0}(x) \\
& +555 G_{0,1,0,1}(x)+32 G_{0,1,0,1}(y)-54 G_{0,1,0,1-x}(y)+777 G_{0,1,1,0}(x) \\
& \text { - } 339 G_{0,1,1,1}(x)+140 G_{0,1,1,1}(y)-84 G_{0,1,1,1-x}(y)-66 G_{0,1,1-x, 0}(y) \\
& -84 G_{0,1,1-x, 1}(y)+30 G_{0,1,1-x, 1-x}(y)-189 G_{0,1-x, 0,0}(y)-60 G_{0,1-x, 0,1}(y) \\
& +453 G_{0,1-x, 0,1-x}(y)-132 G_{0,1-x, 1,1}(y)+90 G_{0,1-x, 1,1-x}(y)+135 G_{0,1-x, 1-x, 0}(y) \\
& +132 G_{0,1-x, 1-x, 1}(y)-105 G_{0,1-x, 1-x, 1-x}(y)-1029 G_{1,0,0,0}(x)+667 G_{1,0,0,1}(x)
\end{aligned}
$$




$$
\begin{aligned}
& -4 G_{1,0,0,1}(y)-18 G_{1,0,0,1-x}(y)+913 G_{1,0,1,0}(x)-459 G_{1,0,1,1}(x)-16 G_{1,0,1,1}(y) \\
& +24 G_{1,0,1,1-x}(y)-30 G_{1,0,1-x, 0}(y)-24 G_{1,0,1-x, 1}(y)+6 G_{1,0,1-x, 1-x}(y) \\
& +36 G_{1,0,-x, 1-x}(y)+1061 G_{1,1,0,0}(x)-667 G_{1,1,0,1}(x)-16 G_{1,1,0,1}(y) \\
& -48 G_{1,1,0,1-x}(y)-801 G_{1,1,1,0}(x)+307 G_{1,1,1,1}(x)+256 G_{1,1,1,1}(y) \\
& -96 G_{1,1,1,1-x}(y)-48 G_{1,1,1-x, 0}(y)-96 G_{1,1,1-x, 1}(y)+24 G_{1,1,1-x, 1-x}(y) \\
& -54 G_{1,1-x, 0,0}(y)-24 G_{1,1-x, 0,1}(y)+30 G_{1,1-x, 0,1-x}(y)-96 G_{1,1-x, 1,1}(y) \\
& +36 G_{1,1-x, 1,1-x}(y)+18 G_{1,1-x, 1-x, 0}(y)+24 G_{1,1-x, 1-x, 1}(y)-6 G_{1,1-x, 1-x, 1-x}(y) \\
& +36 G_{1,1-x,-x, 1-x}(y)-27 G_{1-x, 0,0,0}(y)-12 G_{1-x, 0,0,1}(y)+237 G_{1-x, 0,0,1-x}(y) \\
& +36 G_{1-x, 0,1,1}(y)+18 G_{1-x, 0,1,1-x}(y)+75 G_{1-x, 0,1-x, 0}(y)+36 G_{1-x, 0,1-x, 1}(y) \\
& -153 G_{1-x, 0,1-x, 1-x}(y)-36 G_{1-x, 0,-x, 1-x}(y)+12 G_{1-x, 1,0,1}(y)+30 G_{1-x, 1,0,1-x}(y) \\
& -192 G_{1-x, 1,1,1}(y)+72 G_{1-x, 1,1,1-x}(y)+42 G_{1-x, 1,1-x, 0}(y)+72 G_{1-x, 1,1-x, 1}(y) \\
& -18 G_{1-x, 1,1-x, 1-x}(y)+9 G_{1-x, 1-x, 0,0}(y)-45 G_{1-x, 1-x, 0,1-x}(y)+48 G_{1-x, 1-x, 1,1}(y) \\
& -18 G_{1-x, 1-x, 1,1-x}(y)-3 G_{1-x, 1-x, 1-x, 0}(y)-12 G_{1-x, 1-x, 1-x, 1}(y) \\
& \left.+3 G_{1-x, 1-x, 1-x, 1-x}(y)\right)+\frac{1}{3} \zeta_{3}\left(45 G_{0,0}(x) G_{0}(y)+60 G_{0,0}(x) G_{1}(y)\right. \\
& -186 G_{0,0}(x) G_{1-x}(y)+45 G_{0}(x) G_{0,0}(y)+9 G_{1}(x) G_{0,0}(y)+9 G_{0,1}(x) G_{0}(y) \\
& \text { - } 12 G_{0,1}(x) G_{1}(y)-33 G_{0,1}(x) G_{1-x}(y)+36 G_{0}(x) G_{0,1}(y)-36 G_{1}(x) G_{0,1}(y) \\
& -342 G_{0}(x) G_{0,1-x}(y)-69 G_{1}(x) G_{0,1-x}(y)-93 G_{1,0}(x) G_{0}(y)-72 G_{1,0}(x) G_{1}(y) \\
& -63 G_{1,0}(x) G_{1-x}(y)+6 G_{0}(x) G_{1,0}(y)-12 G_{1}(x) G_{1,0}(y)+123 G_{1,1}(x) G_{0}(y) \\
& +24 G_{1,1}(x) G_{1}(y)-105 G_{1,1}(x) G_{1-x}(y)+48 G_{0}(x) G_{1,1}(y)-48 G_{1}(x) G_{1,1}(y) \\
& -72 G_{0}(x) G_{1,1-x}(y)+24 G_{1}(x) G_{1,1-x}(y)+51 G_{0}(x) G_{1-x, 0}(y)+33 G_{1}(x) G_{1-x, 0}(y) \\
& -30 G_{0}(x) G_{1-x, 1}(y)+30 G_{1}(x) G_{1-x, 1}(y)+48 G_{0}(x) G_{1-x, 1-x}(y)+9 G_{1}(x) G_{1-x, 1-x}(y) \\
& -201 G_{0,0,1-x}(y)-36 G_{0,1,1-x}(y)+87 G_{0,1-x, 0}(y)+153 G_{0,1-x, 1}(y)+87 G_{0,1-x, 1-x}(y) \\
& +6 G_{1,0,1-x}(y)-48 G_{1,1,1-x}(y)-12 G_{1,1-x, 0}(y)+30 G_{1,1-x, 1}(y)+6 G_{1,1-x, 1-x}(y) \\
& +45 G_{1-x, 0,0}(y)+51 G_{1-x, 0,1}(y)+87 G_{1-x, 0,1-x}(y)-18 G_{1-x, 1,0}(y)+60 G_{1-x, 1,1}(y) \\
& +30 G_{1-x, 1,1-x}(y)-15 G_{1-x, 1-x, 0}(y)-15 G_{1-x, 1-x, 1}(y)-9 G_{1-x, 1-x, 1-x}(y) \\
& +45 G_{0,0,0}(x)+9 G_{0,0,1}(x)-93 G_{0,1,0}(x)+123 G_{0,1,1}(x)-161 G_{1,0,0}(x)+107 G_{1,0,1}(x) \\
& +165 G_{1,1,0}(x)-203 G_{1,1,1}(x)+45 G_{0,0,0}(y)-225 G_{0,0,1}(y)-34 G_{0,1,0}(y) \\
& \left.-114 G_{0,1,1}(y)+12 G_{1,0,0}(y)+30 G_{1,0,1}(y)+28 G_{1,1,0}(y)-80 G_{1,1,1}(y)\right) \\
& +\frac{1}{24} \zeta_{4}\left(4401 G_{0}(x) G_{0}(y)-2859 G_{1}(x) G_{0}(y)-78 G_{0}(x) G_{1}(y)+342 G_{1}(x) G_{1}(y)\right. \\
& -81 G_{0}(x) G_{1-x}(y)-15 G_{1}(x) G_{1-x}(y)-1785 G_{0,1-x}(y)+342 G_{1,1-x}(y)-567 G_{1-x, 0}(y) \\
& -126 G_{1-x, 1}(y)+183 G_{1-x, 1-x}(y)+4401 G_{0,0}(x)-2859 G_{0,1}(x)-3755 G_{1,0}(x) \\
& \left.+1987 G_{1,1}(x)+4401 G_{0,0}(y)+278 G_{0,1}(y)+66 G_{1,0}(y)-8 G_{1,1}(y)\right) \\
& +\frac{1}{3} \zeta_{5}\left(-51 G_{1-x}(y)+117 G_{0}(x)-143 G_{1}(x)+117 G_{0}(y)-50 G_{1}(y)\right) \\
& +\frac{1}{3} \zeta_{2} \zeta_{3}\left(27 G_{1-x}(y)+21 G_{0}(x)-73 G_{1}(x)+21 G_{0}(y)+8 G_{1}(y)\right) \\
& +\frac{13777 \zeta_{6}}{96}-\frac{107 \zeta_{3}^{2}}{9} \text {. }
\end{aligned}
$$


Open Access. This article is distributed under the terms of the Creative Commons Attribution License (CC-BY 4.0), which permits any use, distribution and reproduction in any medium, provided the original author(s) and source are credited.

\section{References}

[1] K.G. Chetyrkin and F.V. Tkachov, Integration by parts: the algorithm to calculate $\beta$-functions in 4 loops, Nucl. Phys. B 192 (1981) 159 [INSPIRE].

[2] F.V. Tkachov, A theorem on analytical calculability of four loop renormalization group functions, Phys. Lett. B 100 (1981) 65 [INSPIRE].

[3] R.N. Lee, Group structure of the integration-by-part identities and its application to the reduction of multiloop integrals, JHEP 07 (2008) 031 [arXiv:0804.3008] [INSPIRE].

[4] Z. Bern, L.J. Dixon, D.C. Dunbar and D.A. Kosower, Fusing gauge theory tree amplitudes into loop amplitudes, Nucl. Phys. B 435 (1995) 59 [hep-ph/9409265] [INSPIRE].

[5] R. Britto, F. Cachazo and B. Feng, Generalized unitarity and one-loop amplitudes in $N=4$ super-Yang-Mills, Nucl. Phys. B 725 (2005) 275 [hep-th/0412103] [INSPIRE].

[6] G. Ossola, C.G. Papadopoulos and R. Pittau, Reducing full one-loop amplitudes to scalar integrals at the integrand level, Nucl. Phys. B 763 (2007) 147 [hep-ph/0609007] [INSPIRE].

[7] R. Britto, Loop amplitudes in gauge theories: modern analytic approaches, J. Phys. A 44 (2011) 454006 [arXiv: 1012.4493] [INSPIRE].

[8] R.K. Ellis, Z. Kunszt, K. Melnikov and G. Zanderighi, One-loop calculations in quantum field theory: from Feynman diagrams to unitarity cuts, Phys. Rept. 518 (2012) 141 [arXiv: 1105.4319] [INSPIRE].

[9] J. Butterworth et al., Les Houches 2013: physics at TeV colliders - Standard Model working group report, arXiv: 1405.1067 [INSPIRE].

[10] P. Mastrolia and G. Ossola, On the integrand-reduction method for two-loop scattering amplitudes, JHEP 11 (2011) 014 [arXiv:1107.6041] [INSPIRE].

[11] D.A. Kosower and K.J. Larsen, Maximal unitarity at two loops, Phys. Rev. D 85 (2012) 045017 [arXiv: 1108.1180] [INSPIRE].

[12] S. Badger, H. Frellesvig and Y. Zhang, Hepta-cuts of two-loop scattering amplitudes, JHEP 04 (2012) 055 [arXiv: 1202.2019] [inSPIRE].

[13] Y. Zhang, Integrand-level reduction of loop amplitudes by computational algebraic geometry methods, JHEP 09 (2012) 042 [arXiv: 1205.5707] [INSPIRE].

[14] P. Mastrolia, E. Mirabella, G. Ossola and T. Peraro, Scattering amplitudes from multivariate polynomial division, Phys. Lett. B 718 (2012) 173 [arXiv:1205.7087] [INSPIRE].

[15] P. Mastrolia, E. Mirabella, G. Ossola and T. Peraro, Multiloop integrand reduction for dimensionally regulated amplitudes, Phys. Lett. B 727 (2013) 532 [arXiv:1307.5832] [INSPIRE].

[16] A.V. Kotikov, Differential equations method: new technique for massive Feynman diagrams calculation, Phys. Lett. B 254 (1991) 158 [INSPIRE].

[17] E. Remiddi, Differential equations for Feynman graph amplitudes, Nuovo Cim. A 110 (1997) 1435 [hep-th/9711188] [INSPIRE]. 
[18] T. Gehrmann and E. Remiddi, Differential equations for two loop four point functions, Nucl. Phys. B 580 (2000) 485 [hep-ph/9912329] [INSPIRE].

[19] C. Duhr, Hopf algebras, coproducts and symbols: an application to Higgs boson amplitudes, JHEP 08 (2012) 043 [arXiv: 1203.0454] [INSPIRE].

[20] L.J. Dixon, J.M. Drummond, C. Duhr, M. von Hippel and J. Pennington, Bootstrapping six-gluon scattering in planar $\mathcal{N}=4$ super-Yang-Mills theory, arXiv:1407.4724 [INSPIRE].

[21] S. Laporta, High precision calculation of multiloop Feynman integrals by difference equations, Int. J. Mod. Phys. A 15 (2000) 5087 [hep-ph/0102033] [InSPIRE].

[22] M. Argeri and P. Mastrolia, Feynman diagrams and differential equations, Int. J. Mod. Phys. A $22(2007) 4375$ [arXiv:0707.4037] [InSPIRE].

[23] V.A. Smirnov, Analytic tools for Feynman integrals, Springer Tracts Mod. Phys. 250 (2012) 1 [INSPIRE].

[24] J.M. Henn, Multiloop integrals in dimensional regularization made simple, Phys. Rev. Lett. 110 (2013) 251601 [arXiv: 1304.1806] [INSPIRE].

[25] C.G. Papadopoulos, Simplified differential equations approach for master integrals, JHEP 07 (2014) 088 [arXiv:1401.6057] [INSPIRE].

[26] A. Goncharov, Polylogarithms in arithmetic and geometry, in Proceedings of the International Congress of Mathematicians 1,2 (1995), pg. 374.

[27] J.M. Henn and V.A. Smirnov, Analytic results for two-loop master integrals for Bhabha scattering I, JHEP 11 (2013) 041 [arXiv: 1307.4083] [INSPIRE].

[28] J.M. Henn, A.V. Smirnov and V.A. Smirnov, Evaluating single-scale and/or non-planar diagrams by differential equations, JHEP 03 (2014) 088 [arXiv:1312. 2588] [INSPIRE].

[29] J.M. Henn and T. Huber, The four-loop cusp anomalous dimension in $\mathcal{N}=4$ super Yang-Mills and analytic integration techniques for Wilson line integrals, JHEP 09 (2013) 147 [arXiv: 1304.6418] [INSPIRE].

[30] J.M. Henn, A.V. Smirnov and V.A. Smirnov, Analytic results for planar three-loop four-point integrals from a Knizhnik-Zamolodchikov equation, JHEP 07 (2013) 128 [arXiv:1306.2799] [INSPIRE].

[31] M. Argeri et al., Magnus and Dyson series for master integrals, JHEP 03 (2014) 082 [arXiv: 1401.2979] [INSPIRE].

[32] S. Caron-Huot and J.M. Henn, Iterative structure of finite loop integrals, JHEP 06 (2014) 114 [arXiv: 1404.2922] [INSPIRE].

[33] T. Gehrmann, A. von Manteuffel, L. Tancredi and E. Weihs, The two-loop master integrals for $q \bar{q} \rightarrow V V$, JHEP 06 (2014) 032 [arXiv: 1404.4853] [INSPIRE].

[34] Y. Li, A. von Manteuffel, R.M. Schabinger and H.X. Zhu, $N^{3}$ LO Higgs and Drell-Yan production at threshold: the one-loop two-emission contribution, arXiv:1404.5839 [INSPIRE].

[35] J.M. Henn, K. Melnikov and V.A. Smirnov, Two-loop planar master integrals for the production of off-shell vector bosons in hadron collisions, JHEP 05 (2014) 090 [arXiv: 1402.7078] [INSPIRE].

[36] F. Caola, J.M. Henn, K. Melnikov and V.A. Smirnov, Non-planar master integrals for the production of two off-shell vector bosons in collisions of massless partons, arXiv:1404.5590 [INSPIRE]. 
[37] M. Höschele, J. Hoff and T. Ueda, Adequate bases of phase space master integrals for $g g \rightarrow h$ at NNLO and beyond, arXiv:1407.4049 [INSPIRE].

[38] W. Magnus, On the exponential solution of differential equations for a linear operator, Commun. Pure Appl. Math. 7 (1954) 649 [inSPIRE].

[39] S. Blanes, F. Casas, J.A. Oteo and J. Ros, The magnus expansion and some of its applications, Phys. Rept. 470 (2009) 151 [arXiv:0810.5488].

[40] A.B. Goncharov, Multiple polylogarithms and mixed Tate motives, math.AG/0103059 [INSPIRE].

[41] E. Remiddi and J.A.M. Vermaseren, Harmonic polylogarithms, Int. J. Mod. Phys. A 15 (2000) 725 [hep-ph/9905237] [INSPIRE].

[42] T. Gehrmann and E. Remiddi, Numerical evaluation of harmonic polylogarithms, Comput. Phys. Commun. 141 (2001) 296 [hep-ph/0107173] [InSPIRE].

[43] J. Vollinga and S. Weinzierl, Numerical evaluation of multiple polylogarithms, Comput. Phys. Commun. 167 (2005) 177 [hep-ph/0410259] [INSPIRE].

[44] J. Ablinger, J. Blumlein and C. Schneider, Harmonic sums and polylogarithms generated by cyclotomic polynomials, J. Math. Phys. 52 (2011) 102301 [arXiv:1105.6063] [InSPIRE].

[45] G. Heinrich, T. Huber, D.A. Kosower and V.A. Smirnov, Nine-propagator master integrals for massless three-loop form factors, Phys. Lett. B 678 (2009) 359 [arXiv:0902.3512] [INSPIRE].

[46] G. Heinrich, T. Huber and D. Maître, Master integrals for fermionic contributions to massless three-loop form-factors, Phys. Lett. B 662 (2008) 344 [arXiv:0711.3590] [INSPIRE].

[47] T. Gehrmann, G. Heinrich, T. Huber and C. Studerus, Master integrals for massless three-loop form-factors: one-loop and two-loop insertions, Phys. Lett. B 640 (2006) 252 [hep-ph/0607185] [INSPIRE].

[48] R.N. Lee, A.V. Smirnov and V.A. Smirnov, Analytic results for massless three-loop form factors, JHEP 04 (2010) 020 [arXiv:1001.2887] [INSPIRE].

[49] M. Argeri, P. Mastrolia and E. Remiddi, The analytic value of the sunrise selfmass with two equal masses and the external invariant equal to the third squared mass, Nucl. Phys. B 631 (2002) 388 [hep-ph/0202123] [INSPIRE].

[50] L.W. Garland, T. Gehrmann, E.W.N. Glover, A. Koukoutsakis and E. Remiddi, The two loop QCD matrix element for $e^{+} e^{-} \rightarrow 3$ jets, Nucl. Phys. B 627 (2002) 107 [hep-ph/0112081] [INSPIRE].

[51] L.W. Garland, T. Gehrmann, E.W.N. Glover, A. Koukoutsakis and E. Remiddi, Two loop QCD helicity amplitudes for $e^{+} e^{-} \rightarrow 3$ jets, Nucl. Phys. B 642 (2002) 227 [hep-ph/0206067] [INSPIRE].

[52] A. Gehrmann-De Ridder, T. Gehrmann, E.W.N. Glover and G. Heinrich, NNLO moments of event shapes in $e^{+} e^{-}$annihilation, JHEP 05 (2009) 106 [arXiv:0903.4658] [INSPIRE].

[53] S. Weinzierl, Event shapes and jet rates in electron-positron annihilation at NNLO, JHEP 06 (2009) 041 [arXiv:0904.1077] [INSPIRE].

[54] T. Gehrmann, M. Jaquier, E.W.N. Glover and A. Koukoutsakis, Two-loop QCD corrections to the helicity amplitudes for $H \rightarrow 3$ partons, JHEP 02 (2012) 056 [arXiv:1112.3554] [INSPIRE]. 
[55] R. Boughezal, F. Caola, K. Melnikov, F. Petriello and M. Schulze, Higgs boson production in association with a jet at next-to-next-to-leading order in perturbative QCD, JHEP 06 (2013) 072 [arXiv: 1302.6216] [INSPIRE].

[56] S.D. Badger and E.W.N. Glover, Two loop splitting functions in QCD, JHEP 07 (2004) 040 [hep-ph/0405236] [INSPIRE].

[57] D.A. Kosower and P. Uwer, Evolution kernels from splitting amplitudes, Nucl. Phys. B 674 (2003) 365 [hep-ph/0307031] [INSPIRE].

[58] T. Gehrmann and E. Remiddi, Numerical evaluation of two-dimensional harmonic polylogarithms, Comput. Phys. Commun. 144 (2002) 200 [hep-ph/0111255] [InSPIRE].

[59] T. Gehrmann and E. Remiddi, Two loop master integrals for $\gamma^{*} \rightarrow 3$ jets: the planar topologies, Nucl. Phys. B 601 (2001) 248 [hep-ph/0008287] [INSPIRE].

[60] C. Studerus, Reduze - Feynman integral reduction in C ++ , Comput. Phys. Commun. 181 (2010) 1293 [arXiv: 0912. 2546] [InSPIRE].

[61] A. von Manteuffel and C. Studerus, Reduze 2 - distributed Feynman integral reduction, arXiv: 1201.4330 [INSPIRE].

[62] C.W. Bauer, A. Frink and R. Kreckel, Introduction to the GiNaC framework for symbolic computation within the $C++$ programming language, cs/0004015 [INSPIRE].

[63] A.V. Smirnov and M.N. Tentyukov, Feynman Integral Evaluation by a Sector decomposiTion Approach (FIESTA), Comput. Phys. Commun. 180 (2009) 735 [arXiv:0807.4129] [INSPIRE].

[64] A.V. Smirnov, FIESTA 3: cluster-parallelizable multiloop numerical calculations in physical regions, Comput. Phys. Commun. 185 (2014) 2090 [arXiv:1312.3186] [InSPIRE].

[65] T. Gehrmann and E. Remiddi, Analytic continuation of massless two loop four point functions, Nucl. Phys. B 640 (2002) 379 [hep-ph/0207020] [INSPIRE]. 